\title{
CLASSIFICATION OF AFFINE VORTICES
}

\author{
S. VENUGOPALAN AND C. WOODWARD
}

\begin{abstract}
We prove a Hitchin-Kobayashi correspondence for vortices on the complex affine line with Kähler target, which generalizes a result of Taubes [40], see also [24], for the case of a line target. More precisely, suppose a compact connected Lie group $K$ acts on a Kähler manifold $X$ with proper moment map so that stable=semistable for the action of the complexified Lie group $G$ and $X$ is equivariantly convex at infinity. Then, for some sufficiently divisible integer $n$, there is a bijection between gauge equivalence classes of $K$-vortices with target $X$ modulo gauge and isomorphism classes of maps from the weighted projective line $\mathbb{P}(1, n)$ to $X / G$ that map the stacky point at infinity $\mathbb{P}(n)$ to the semistable locus of $X$. The results allow the construction and partial computation of the quantum Kirwan map in Woodward ([46], [47] and [48]), and play a role in the conjectures of Dimofte, Gukov, and Hollands [13] relating vortex counts to knot invariants.
\end{abstract}

\section{Contents}

1. Introduction 2

2. Background 6

2.1. Hamiltonian manifolds 6

2.2. Connections and curvature $\quad 7$

2.3. Complex gauge transformations $\quad 7$

2.4. Gauged holomorphic maps 8

2.5. Gauge theory on weighted projective lines 9

2.6. Standard form near infinity 11

$\begin{array}{ll}\text { 2.7. Equivariant convexity } & 13\end{array}$

$\begin{array}{ll}\text { 2.8. Sobolev Spaces } & 13\end{array}$

3. From holomorphic maps to vortices 14

3.1. Statement of main theorem 14

$\begin{array}{ll}3.2 \text {. The heat flow } & 15\end{array}$

$\begin{array}{ll}\text { 3.3. } & \text { Elliptic regularity for gauged holomorphic maps } \\ \end{array}$

$\begin{array}{ll}\text { 3.4. } & \text { Proof of Theorem 3.1(a) } 20\end{array}$

3.5. Producing a limit modulo bubbling 21

3.6. Finding complex gauge transformations to relate nearby vortices $\quad 27$

3.7. Some results involving complex gauge transformations 30

3.8. A technical result 31

4. From vortices to holomorphic maps 34

4.1. Proof of Theorem 3.1 (b) 34

4.2. Unique affine vortex in a complex gauge orbit 39

2010 Mathematics Subject Classification. 53D06. 
5. Asymptotic decay for vortices 42

6. Some analytic results 44

$\begin{array}{ll}\text { References } & 46\end{array}$

\section{INTRODUCTION}

A vortex in geometric analysis is a pair consisting of a connection and bundle section which satisfies an equation involving the curvature and a non-linear (usual quadratic) term depending on the section, as well as a first order equation for the section. Vortices arise naturally in several areas of mathematical physics and geometry; our interest in them arises from their interpretation as equivariant generalizations of pseudoholomorphic maps. Our goal in this paper is to classify vortices, in the special case that the domain is the complex line. In this setting, Taubes [40] and Jaffe-Taubes [24] provide a classification in the first non-trivial case of finite energy vortices with circle group $K=U(1)$ and target space $X=\mathbb{C}$. In this paper we generalize their classification to arbitrary compact connected Lie groups $K$ and fiber bundles whose fiber is a Kähler manifold $X$ with a Hamiltonian $K$ action. Here $X$ is either compact or is equivariantly convex at infinity with a proper moment map. More precisely we classify affine vortices with target $X$, consisting of pairs $(A, u)$ where $A$ is a connection on the principal bundle $P=\mathbb{C} \times K$, $u: \mathbb{C} \rightarrow P(X):=(P \times X) / K$ is a holomorphic section with respect to the complex structure defined by $A$ and $(A, u)$ satisfies the vortex equation:

$$
* F_{A}+\Phi(u)=0 \text {. }
$$

Here $F_{A}$ is the curvature of $A, \Phi: X \rightarrow \mathfrak{k}^{\vee} \simeq \mathfrak{k}$ is the moment map on $X$ for the $K$-action, and $*$ is the Hodge star for the standard metric on $\mathbb{C}$.

In the special case of the action of the circle group on the complex line, Taubes [40, Theorem 1]) and Jaffe-Taubes [24, Section III, Theorem 1] show that the gaugeequivalence class of a finite energy vortex is completely determined by the zeros of the section. It follows that the moduli space of gauge equivalence classes of finite energy vortices is a symmetric product. This gives a description of the space of vortices with target $\mathbb{C}$ in terms of holomorphic data which from a more modern perspective may be viewed as a Hitchin-Kobayashi correspondence. The first example of such a correspondence by Narasimhan and Seshadri [32] shows that stable holomorphic bundles over a Riemann surface correspond to irreducible unitary representations of the fundamental group. Donaldson [14] reproves the Narasimhan-Seshadri theorem in a differential-geometric setting, replacing irreducible unitary representations by an equivalent object - the minima of the Yang-Mills functional. The extension of this result to higher dimensional base manifolds involved replacing a Yang-Mills connection by a Hermitian-Einstein connection. The Hitchin-Kobayashi correspondence in this case states that a holomorphic vector bundle admits a Hermitian-Einstein connection if and only if it is stable. This was proved for compact Kähler surfaces in [15] and for general compact Kähler manifolds in [41]. 
In the case of vortices the corresponding holomorphic objects are holomorphic bundles over compact Kähler manifolds with additional data, for example a prescribed holomorphic section. Vortices are the zeros of the vortex functional given by the norm-square of the left hand side of (1). Bradlow's paper [7] defines a stability condition for such objects and relates it to the existence of zeros of the vortex functional. Results in Bradlow [7] are used to investigate the moduli space of finite energy vortices in [8], [5] and [6]. Garcia-Prada [19], [20], [21] provides a different approach to similar results via dimensional reduction, achieving in some cases more general results. Mundet [31] generalizes these results by allowing the fiber to be a Kähler Hamiltonian manifold. All these Hitchin-Kobayashi results are infinite-dimensional versions of the abstract setting laid out in Kempf-Ness [25] and Kirwan [27], in which the main idea is that the symplectic quotient coincides with the geometric invariant theory (git) quotient.

Our main result is a bijection between finite energy affine vortices with bounded image and a moduli space of holomorphic objects defined as follows: Let $G$ be a connected complex reductive group whose maximal compact subgroup is $K$. By a $G$-gauged holomorphic map we mean a pair $\left(P_{\mathbb{C}}, u\right)$ where $P_{\mathbb{C}} \rightarrow \mathbb{P}(1, n)$ is a holomorphic principal $G$-bundle over the weighted projective line $\mathbb{P}(1, n)$ and $u: \mathbb{P}(1, n) \rightarrow$ $P_{\mathbb{C}} \times_{G} X$ is a section. Complex gauge transformations are $G$-automorphisms of the underlying smooth bundle; they act on $G$-gauged maps by pulling back the complex structure and section. We say that a $G$-gauged holomorphic map is stable if the point at infinity is mapped by $u$ to the semistable locus $X^{\text {ss }}$, i.e., $u(\infty)$ takes values in $\left.\left(P_{\mathbb{C}} \times_{G} X^{\mathrm{ss}}\right)\right|_{\infty}$. The stability condition we consider here is surprisingly simple because we want our holomorphic objects to correspond to unitary objects on affine curves. From a $G$-gauged holomorphic map $\left(P_{\mathbb{C}}, u\right)$, we produce a unitary object by choosing a section $\sigma: \mathbb{P}(1, n) \rightarrow P_{\mathbb{C}} / K$. This reduces the structure group to the maximal compact subgroup $K$, i.e. there is a principal $K$-bundle $P \subseteq P_{\mathbb{C}}$ such that $P_{\mathbb{C}}=P \times_{K} G$. The complex structure on $P_{\mathbb{C}}$ corresponds to a choice of $K$-connection $A$.

Theorem 1.1. (Classification of affine vortices) Let $X, G, K$ be as above. Suppose the $G$-action on $X^{\mathrm{ss}}$ has finite stabilizers and $n$ is a positive integer such that for any $x \in X^{\mathrm{ss}}$, the order of the stabilizer group $\left|G_{x}\right|$ divides $n$. For any stable $G$ gauged map $\left(P_{\mathbb{C}}, u\right)$, there exists a unique reduction of the structure group $\sigma: \mathbb{C} \rightarrow$ $P_{\mathbb{C}} / K$, so that if $A$ is the resulting connection, then $\left.(A, u)\right|_{\mathbb{C}}$ is a symplectic vortex. The reduction $\sigma$ extends over $\mathbb{P}(1, n)$ in the sense of Section 3 below. The map $\left[\left(P_{\mathbb{C}}, u\right)\right] \mapsto\left[\left.(A, u)\right|_{\mathbb{C}}\right]$ defines a bijection between complex gauge equivalence classes of stable gauged holomorphic maps $\left(P_{\mathbb{C}}, u\right)$ from $\mathbb{P}(1, n)$ to $X$ and gauge equivalence classes of finite energy affine $K$-vortices with bounded image in $X$.

The moduli space of $G$-gauged holomorphic maps in the theorem has a convenient stack-theoretic interpretation. The weighted projective line is a Deligne-Mumford stack, or in other language, an orbifold. Recall that if $C$ is a complex curve, a morphism from $C$ to the quotient stack $X / G$ consists of a principal $G$-bundle over $C$ together with a section of the associated fiber bundle $P \times_{G} X$. The quotient stack 
$X / G$ contains as a proper open substack the geometric invariant theory quotient $X / / G$, here defined as the stack-theoretic quotient of the semistable locus by the action of $G$. The same is true when $C$ is replaced by $\mathbb{P}(1, n)$ (see [28]). The moduli space of stable $G$-gauged holomorphic maps is the coarse moduli space of the open substack of $\operatorname{Hom}(\mathbb{P}(1, n), X / G)$ that satisfies $u(\mathbb{P}(n)) \subset X / / G$. In the case that $G$ is a torus acting on a finite dimensional complex vector space $X$, bundles on $\mathbb{P}(1, n)$ and sections of the associated vector bundle can be classified explicitly, see Proposition 1.2 below.

A recent paper of $\mathrm{Xu}$ [49] complements our work. It proves similar results as this paper for $U(1)$-vortices with fiber $X=\mathbb{C}^{m}$ using results of [7]. It also shows a correspondence between compactifications of the space of affine vortices modulo gauge on the one side and the space of gauged holomorphic maps over $\mathbb{P}^{1}$, that are semistable at $\infty$.

The Hitchin-Kobayashi correspondence for affine vortices in Theorem 1.1 is partly motivated by a certain quantization of the Kirwan map that arises in the study of Gromov-Witten invariants of geometric invariant theory quotients. Namely Kirwan $[27]$ constructs a map from the equivariant cohomology $H_{G}(X, \mathbb{Q})$ to the cohomology of the quotient $H(X / / G, \mathbb{Q})$. A Gromov-Witten generalization of [27] called the quantum Kirwan map, suggested by Gaio-Salamon [18], maps the equivariant quantum cohomology of $X$ to the quantum cohomology of the quotient $X / / G$. Salamon and Ziltener [50] suggested to define the quantum Kirwan map by a count of affine vortices. The papers of the second author [33], [46], [47] and [48] generalize the manifolds for which this map is defined, by removing monotonicity and asphericity assumptions on $X$. Also, the group action can have finite stabilizers on the zero-level set of the moment map, i.e. the symplectic quotient can be an orbifold. This paper is part of that project. It provides an algebraic description for the moduli space of vortices, and from there, the quantum Kirwan map can be defined using BehrendFantechi machinery. A very important result in this context is a compactification for the space of affine vortices modulo gauge proved by Ziltener ([50], [51]).

An additional recent motivation arises from knot-invariants via vortex counting conjectures of Dimofte, Gukov, and Hollands [13]. In these conjectures, the equivariant index (defined via localization) of the moduli space of affine vortices is conjectured to be a certain knot invariant. Our results allow the identification of the moduli space of affine vortices with the quasimap spaces discussed in, for example, Bertram, Ciocan-Fontanine, and Kim [3]. The space of matrix-valued vortices in Example 1.3 appears as the relevant space of vortices for a torus knot in the vortex counting conjectures of [13].

The proof of the Hitchin-Kobayashi correspondence for affine vortices uses a minimization technique for the vortex functional. In particular the first author proved in [43] that the heat flow of the functional

$$
(A, u) \mapsto\left\|* F_{A}+\Phi(u)\right\|_{L^{2}(\Sigma)}
$$

exists for all time on any compact Riemann surface $\Sigma$, possibly with boundary. Using this result, the vortex equation on an affine line can be solved on a succession 
of annuli of increasing sizes that exhaust the domain. We prove that this sequence of vortices converges to a solution on the whole plane. This process involves a number of complications, such as proving that the sequence of solutions on the balls converges without bubbling.

We give some examples of the main result in the case of torus actions on vector spaces. By Theorem 3.1, given a finite energy affine vortex $(A, u)$ with bounded image, it corresponds to a gauged holomorphic map over a principal $K$-bundle $P \rightarrow \mathbb{P}(1, n)$ in a sense defined in Section 3.1. The characteristic class $[P]$ of $P$ in $H_{2}(B K, \mathbb{Q})$ is a topological invariant of $(A, u)$ (see Remark 2.5). If $K$ is a torus then $H_{2}(B K, \mathbb{Q})$ is isomorphic to the subset $\left\{\lambda \in \mathfrak{k} \mid \exists n \in \mathbb{Z}, e^{2 \pi n \lambda}=\operatorname{Id}\right\}$ of rational elements $\mathfrak{k}$ and we call the image of $[P]$ in $\mathfrak{k}$ the degree of the vortex. In the following Proposition, we classify affine vortices of fixed degree.

Proposition 1.2. (Classification of affine vortices in the toric case) Suppose that $G$ is a complex torus acting on a complex vector space $X=\mathbb{C}^{k}$ with weights $\nu_{1}, \ldots, \nu_{k} \in$ $\mathfrak{g}^{\vee}$ contained in an open half space, and spanning $\mathfrak{g}^{\vee}$. Then, under the bijection in Theorem 1.1 affine vortices of degree $d \in \mathfrak{k} \subset \mathfrak{g}$ correspond to isomorphism classes of tuples of polynomial maps $u=\left(u_{1}, \ldots, u_{k}\right): \mathbb{C} \rightarrow X$ satisfying

(a) the degree of $u_{j}$ is at most $\left\langle\nu_{j}, d\right\rangle$ for each $j=1, \ldots, k$; and

(b) if

$$
u(\infty):=\left(u_{j}(\infty):=\left\{\begin{array}{ll}
u_{j}^{\left(\left\langle\nu_{j}, d\right\rangle\right)} /\left\langle\nu_{j}, d\right\rangle ! & \left\langle\nu_{j}, d\right\rangle \in \mathbb{Z} \\
0 & \text { otherwise }
\end{array}\right)_{j=1}^{k}\right.
$$

denotes the vector of leading order coefficients (here $u_{j}^{\left(\left\langle\nu_{j}, d\right\rangle\right)}$ denotes the $\left\langle\nu_{j}, d\right\rangle$-th derivative of $\left.u_{j}\right)$ with integer exponents, then $u(\infty) \in X^{\mathrm{ss}}$.

Two such tuples are isomorphic if they are related by the action of $G$.

We defer the proof to the end of Section 4.

Example 1.3. (a) (Linear vortices) If $X=\mathbb{C}$ with $\nu_{1}=1$, then affine vortices of class $d$ correspond to polynomials of degree exactly $d$ up to the action of scalar multiplication, hence classified by their zeroes. This recovers the Taubes [40], [24] result.

(b) (Matrix-valued vortices and Weil's torsion quotients) If $X=M_{n}(\mathbb{C})$, the space of $n \times n$ matrices, and $G=G L_{n}$ acts by left multiplication, then the semistable locus consists of invertible matrices and the action on the semistable locus is free. Theorem 3.1 gives a classification of vortices according to the following data: By Grothendieck's theorem [22], any holomorphic vector bundle on $\mathbb{P}^{1}$ splits as a sum of line bundles

$$
P \times_{G L_{n}(\mathbb{C})} \mathbb{C}^{n} \cong \mathcal{O}_{\mathbb{P}^{1}}\left(\lambda_{1}\right) \oplus \ldots \oplus \mathcal{O}_{\mathbb{P}^{1}}\left(\lambda_{n}\right), \quad \lambda_{1} \geq \ldots \geq \lambda_{n}
$$

where $\mathcal{O}_{\mathbb{P}^{1}}(\lambda)$ is the $\lambda$-th tensor power of the hyperplane bundle. The associated $X$ bundle is then $P(X)=\mathcal{O}_{\mathbb{P}^{1}}\left(\lambda_{1}\right)^{\oplus n} \oplus \ldots \oplus \mathcal{O}_{\mathbb{P}^{1}}\left(\lambda_{n}\right)^{\oplus n}$. A section $u$ of $P(X)$ may be viewed as a matrix-valued function on $\mathbb{C}$. The semistability condition at infinity is then the condition that the leading order terms of 
$u$ form an invertible matrix. Thus $u$ defines a morphism of sheaves which is generically an isomorphism, providing a connection to Weil's scheme of torsion quotients considered in [3], [4], which [3] mentions was considered by Weil to be the non-abelian analog of the symmetric product of the curve.

We briefly sketch the contents of the paper. Section 2 defines gauged holomorphic maps and extends the definitions to the case when the base manifold $\Sigma$ is an orbifold $\mathbb{P}(1, n)$. Section 3 gives an analytic version of the main theorem and proves the first part of the main theorem 3.1. Section 4 proves the second part. The proof relies on removal of singularities at infinity for finite energy vortices with bounded image which is proved in Section 5.

Acknowledgments: We thank Fabian Ziltener for useful discussions. S.V. was a post-doctoral fellow at Tata Institute of Fundamental Research, Bombay at the time this paper was written. C.W. was partially supported by NSF grant DMS 1207194 and a Simons Fellowship. We also acknowledge HIM, Bonn for hosting us for a week, when some of the work on this paper was carried out. Finally, we profusely thank the referee for reading the paper carefully and providing suggestions that improved the paper.

\section{BACKGROUND}

In this section we introduce basic notation for gauged holomorphic maps and vortices, especially gauged holomorphic maps on weighted projective lines.

2.1. Hamiltonian manifolds. We introduce the following notation for group actions. Let $G$ be a complex reductive Lie group with maximal compact subgroup $K$, so that $G$ is the complexification of $K$. Let $(X, \omega, J)$ be a Kähler manifold with symplectic structure $\omega$ and complex structure $J$ on which $G$ acts holomorphically and $K$ acts symplectically. A moment map is a $K$-equivariant map $\Phi: X \rightarrow \mathfrak{k}^{\vee}$ such that

$$
\iota\left(\xi_{X}\right) \omega=\mathrm{d}\langle\Phi, \xi\rangle, \forall \xi \in \mathfrak{k},
$$

where $\xi_{X}(x)=\left.\frac{d}{d t} \exp (t \xi) x\right|_{t=0} \in \operatorname{Vect}(X)$ is given by the infinitesimal action of $\xi$ on $X$. The action of $K$ is Hamiltonian if there exists a moment map $\Phi: X \rightarrow \mathfrak{k}^{\vee}$. Since $K$ is compact, $\mathfrak{k}$ has an Ad-invariant metric. We fix such a metric and identify $\mathfrak{k} \simeq \mathfrak{k}^{\vee}$, so the moment map becomes a map $\Phi: X \rightarrow \mathfrak{k}$. We assume $X$ is equipped with a Hamiltonian action and fix the moment map. In the rest of the paper, we assume:

Assumption 2.1. The G-action on $G \Phi^{-1}(0)$ has finite stabilizers.

If the underlying complex structure of $X$ has the structure of a polarized projective or affine $G$-variety, the git quotient is defined by $X / / G:=X^{\mathrm{ss}} / \sim$ where $X^{\mathrm{ss}}$ is the semi-stable locus and $\sim$ is the orbit closure relation. For details of the construction, refer to [30] for the projective case and [26] for the affine case. A polarization of $X$ determines a moment map. By the Kempf-Ness [25] theorem and its generalization 
to affine varieties $([26$, Theorem 6.1$])$, the quotient $X / / G$ is homeomorphic to the symplectic quotient $\Phi^{-1}(0) / K$. Assumption 2.1 guarantees that the $K$ action on $\Phi^{-1}(0)$ has finite stabilizers, and that $X^{\mathrm{ss}}=G \Phi^{-1}(0)$. In case $X$ is a Kähler manifold without an algebraic structure, we define the semistable locus as $X^{\text {ss }}:=$ $G \Phi^{-1}(0)$.

2.2. Connections and curvature. We begin with basic notions of connections, curvature, and their behavior under gauge transformations. Let $\Sigma$ be a Riemann surface, equipped with a complex structure $j_{\Sigma}: T \Sigma \rightarrow T \Sigma$ and a volume form $\omega_{\Sigma} \in$ $\Omega^{2}(\Sigma)$ that induces a metric. Let $P \rightarrow \Sigma$ be a principal $K$-bundle. A connection is a $K$-equivariant one-form $A \in \Omega^{1}(P, \mathfrak{k})^{K}$, that satisfies $A\left(\xi_{P}\right)=\xi$ for $\xi \in \mathfrak{k}$. The space of connections $\mathcal{A}(P)$ is an affine space modelled on $\Omega^{1}(\Sigma, P(\mathfrak{k}))$, where $P(\mathfrak{k})=P \times_{K} \mathfrak{k}$ is the adjoint bundle. In case $P$ is the trivial bundle $\Sigma \times K$, there is a trivial connection $\mathrm{D}$, and the adjoint bundle has a trivialization $P(\mathfrak{k}) \simeq \Sigma \times \mathfrak{k}$. Then, the space of connections is

$$
\mathcal{A}(P)=\mathrm{D}+\Omega^{1}(\Sigma, \mathfrak{k}) .
$$

The curvature of a connection $A$ is a two-form $F_{A} \in \Omega^{2}(\Sigma, P(\mathfrak{k}))$. In particular, on a trivial bundle, for a connection $A=\mathrm{D}+a$,

$$
F_{A}:=\mathrm{d} a+[a \wedge a] / 2 \in \Omega^{2}(\Sigma, \mathfrak{k}) .
$$

The curvature varies with the connection as

$$
F_{A+t a}=F_{A}+t \mathrm{~d}_{A} a+\frac{t^{2}}{2}[a \wedge a]
$$

where $\mathrm{d}_{A}$ is the associated covariant derivative on the adjoint bundle. A gauge transformation is an automorphism of $P$ that is an equivariant bundle map $P \rightarrow P$. Alternatively, it is a section of the bundle $P \times_{K} K \rightarrow \Sigma$, where $K$ acts on itself by conjugation. The group of gauge transformations on $P$ is denoted $\mathcal{K}(P)$. On the trivial bundle $\Sigma \times K$, an element in $\mathcal{K}(P)$ is a map from $\Sigma$ to $K$. An element $k \in \mathcal{K}(P)$ acts on a connection $A=\mathrm{D}+a$ as

$$
k(A)=\mathrm{D}+\left(k \mathrm{~d} k^{-1}+\operatorname{Ad}_{k} a\right) .
$$

Differentiating, we see that the infinitesimal action of $\xi: \Sigma \rightarrow \mathfrak{k}$ on $A$ is $-\mathrm{d}_{A} \xi$.

2.3. Complex gauge transformations. We introduce notation for complex gauge transformations. Associated to the principal $K$-bundle $P \rightarrow \Sigma$, we have a principal $G$-bundle $P_{\mathbb{C}}:=P \times_{K} G$ on $\Sigma$. A complex gauge transformation is a $G$-equivariant bundle automorphism $P_{\mathbb{C}} \rightarrow P_{\mathbb{C}}$. The group of complex gauge transformations is denoted by $\mathcal{G}(P)$. It is equivalent to the space of sections $g: \Sigma \rightarrow P \times_{K} G$, where $K$ acts on $G$ by conjugation. Recall that

$$
K \times \mathfrak{k} \rightarrow G, \quad(k, s) \mapsto k e^{i s}
$$

is a diffeomorphism (see Helgason [23, VI.1.1]). So, a complex gauge transformation $g$ can be written as $g=k e^{i \xi}$, where $k \in \mathcal{K}(P)$ and $\xi \in \operatorname{Lie}(\mathcal{K}(P))=\Gamma(P(\mathfrak{k}))$. We next explain the action of $\mathcal{G}(P)$ on the space of connections $\mathcal{A}(P)$. 
Unitary connections determine holomorphic structures on fiber bundles as follows. For any $K$-manifold $X$ we have an associated fiber bundle $P(X)$ over $\Sigma$, and the connection $A$ defines a connection on $P(X)$ and hence a covariant derivative

$$
\mathrm{d}_{A}: \Gamma(P(X)) \rightarrow \bigcup_{u \in \Gamma(P(X))} \Omega^{1}\left(\Sigma, u^{*} T^{\mathrm{vert}}(P(X))\right),
$$

where $T^{\mathrm{vert}}(P(X))$ is the vertical part of the tangent bundle. For example on the bundle $\Sigma \times X$, writing $A=\mathrm{D}+a$,

$$
\mathrm{d}_{A} u:=\mathrm{d} u+a_{u} \in \Omega^{1}\left(\Sigma, u^{*} T X\right) .
$$

At a point $z \in \Sigma, a_{u}(z)$ is the infinitesimal action of $a(z)$ at $u(z)$. If the fiber $X$ is also a complex manifold so that the $K$-action is holomorphic, then one obtains a holomorphic structure on the associated fiber bundle $P(X)$ by defining the the $\bar{\partial}_{A}$ operator by

$$
\bar{\partial}_{A}: \Gamma(\Sigma, P(X)) \rightarrow \Omega^{0,1}\left(\Sigma, u^{*} T^{\mathrm{vert}} P(X)\right), \quad u \mapsto\left(\mathrm{d}_{A} u\right)^{0,1} .
$$

In particular, taking $X=G$ produces a holomorphic $G$-bundle $P_{\mathbb{C}}$. Let $\mathcal{C}(P)$ denote the space of holomorphic structures on $P_{\mathbb{C}}$. The above construction yields a map from $\mathcal{A}(P)$ to $\mathcal{C}(P)$. Conversely, given a holomorphic bundle $P_{\mathbb{C}}$, a choice of a section $\sigma: \Sigma \rightarrow P_{\mathbb{C}} / K$ gives a principal $K$-bundle $P$ by pullback of the bundle $P_{\mathbb{C}} \rightarrow P_{\mathbb{C}} / K$, so that $P$ is naturally a submanifold of $P_{\mathbb{C}}$. The intersection $T P \cap J(T P)$ defines a connection in $T P$ [37]. The correspondence between connections and holomorphic structures gives an infinitesimal isomorphism

$$
T_{A} \mathcal{A}=\Omega^{1}(\Sigma, P(\mathfrak{k})) \rightarrow T_{C} \mathcal{C}=\Omega^{0,1}(\Sigma, P(\mathfrak{g})), \quad a \mapsto a^{0,1} .
$$

The complex structure on $\mathcal{C}$ pulls back to a complex structure on $\mathcal{A}$ given by $J_{\mathcal{A}} a=$ $a \circ j_{\Sigma}$.

The identification between complex structures and connections is equivariant for gauge transformations. The group of complex gauge transformations $\mathcal{G}(P)$ acts on the space of holomorphic structures $\mathcal{C}$ on $P_{\mathbb{C}}$ by pull-back, and pulling back to $\mathcal{A}(P)$ one obtains an action of $\mathcal{G}(P)$ on $\mathcal{A}(P)$ which extends the action of unitary gauge transformations $\mathcal{K}(P)$. For any $\xi \in \Gamma(\Sigma, P(\mathfrak{k}))$, the infinitesimal action of $i \xi$ on $A$ is $-\mathrm{d}_{A} \xi \circ j_{\Sigma}$. Since $\Sigma$ is a Riemann surface, the infinitesimal action of $\xi$ can be rewritten as

$$
(i \xi)_{\mathcal{A}(P)}=-\mathrm{d}_{A} \xi \circ j_{\Sigma}=* \mathrm{~d}_{A} \xi
$$

2.4. Gauged holomorphic maps. Roughly speaking "gauging" an object means introducing a connection into the picture. A gauged holomorphic map $(A, u)$ from $P$ to $X$ consists of a connection $A$ and a section $u$ of $P(X)$ that is holomorphic with respect to $\bar{\partial}_{A}$. The space of gauged holomorphic maps from $P$ to $X$ is denoted $\mathcal{H}(P, X)$. We remark that here we are talking about $K$-gauged holomorphic maps, which will be the default for this paper, as opposed to the $G$-gauged holomorphic map defined in Section 1. A symplectic vortex is a gauged holomorphic map that satisfies

$$
F_{A, u}:=* F_{A}+\Phi(u)=0 .
$$


In the case when $\Sigma=\mathbb{C}$ is equipped with the standard Euclidean metric, a solution of (7) is called an affine vortex. The energy of a gauged holomorphic map $(A, u)$ is

$$
E(A, u):=\int_{\Sigma}\left(\left|F_{A}\right|^{2}+\left|\mathrm{d}_{A} u\right|^{2}+|\Phi \circ u|^{2}\right) \omega_{\Sigma} .
$$

For $\Sigma=\mathbb{C}$, finite energy symplectic vortices with bounded image have good asymptotic properties (see [18, Section 11], [51]). The complexified gauge group acts on $\mathcal{H}(P, X)$ diagonally: $g(A, u):=(g(A), g u)$. This action preserves holomorphicity (see [43]) but not the vortex equation unless the gauge transformation is unitary. From the holomorphic viewpoint, a $K$-gauged holomorphic map $(A, u)$ on a Riemann surface $\Sigma$ consists of a $G$-gauged holomorphic map $\left(P_{\mathbb{C}}, u\right)$ and a section $\sigma: \Sigma \rightarrow P_{\mathbb{C}} / K$. The section $\sigma$ provides a reduction of the structure group $G$ to $K$ and yields a $K$-bundle $P \subset P_{\mathbb{C}}$. The holomorphic structure on $P_{\mathbb{C}}$ determines a connection $A$ on $P$. This point of view is used in stating Theorem 1.1.

2.5. Gauge theory on weighted projective lines. In the Hitchin-Kobayashi correspondence we wish to prove, gauged holomorphic maps on orbifolds play an important role. For orbifolds, we follow classical definitions of Satake [36]. In Satake's definition, composition of morphisms is ambiguous, but that issue does not arise in our paper. Gauged holomorphic maps on orbifolds are similar to $J$-holomorphic curves on orbifolds described in [9]. We will not repeat full definitions here, but just describe what the definitions give in the specific case that the orbifold is the weighted projective line. We begin with some notation for weighted projective lines. For $n$ a positive integer, $\mathbb{P}(1, n)$ is the quotient of $\mathbb{C}^{2}-\{0\}$ by the action of $\mathbb{C}^{*}$ with weights $1, n$. It is covered by two orbifold charts, $\tilde{U}_{1}$ and $U_{2}$ where $\tilde{U}_{1}=U_{2}=\mathbb{C}$ mapping to $\mathbb{P}(1, n)$ as $z \mapsto[(1, z)]$ and $z \mapsto[(z, 1)]$ respectively. The equivalences are :

$$
\begin{array}{rl}
z \sim e^{2 \pi i / n} z & z \in \tilde{U}_{1} \\
z^{-n} \sim w & 0 \neq w \in U_{2}, 0 \neq z \in \tilde{U}_{1} .
\end{array}
$$

We refer to $\tilde{U}_{1} / \sim$ as $U_{1}$. We denote by $\sigma_{n}: \tilde{U}_{1} \rightarrow \tilde{U}_{1}, z \mapsto e^{2 \pi i / n} z$ the diffeomorphism giving the action of the generator of $\mathbb{Z}_{n}$. The orbifold $\mathbb{P}(1, n)$ has smooth locus given by a copy of $\mathbb{C}$, and if $n \neq 1$ has an "orbifold singularity" at $\infty$. More precisely, the orbifold point is the quotient $\mathbb{P}(n)$ of $\mathbb{C}^{*}$ by $\mathbb{C}^{*}$ acting with weight $n$. As a groupoid this is equivalent to the quotient of a point by $\mathbb{Z}_{n}$, that is, $B \mathbb{Z}_{n}$.

Principal bundles on the weighted projective line can be defined via the clutching construction as follows. For a more general definition of bundles on orbifolds, see [2]. An orbifold principal $K$-bundle $P$ on $\mathbb{P}(1, n)$ is given by transition maps $\mu: \tilde{U}_{1} \rightarrow K$ and $\tau: \tilde{U}_{1} \backslash\{0\} \rightarrow K$ that satisfy $\tau\left(\sigma_{n}(z)\right)=\tau(z) \mu(z)^{-1}$.

$$
P:=\left(\left(\tilde{U}_{1} \times K\right) \bigsqcup\left(U_{2} \times K\right)\right) / \sim,
$$

and the equivalence $\sim$ is given by

$$
\begin{aligned}
& (z, h) \sim\left(\sigma_{n} z, \mu(z) h\right) \quad(z, h) \in \tilde{U}_{1} \times K \\
& (z, h) \sim(w, \tau(z) h) \quad 0 \neq z \in \tilde{U}_{1}, w \in U_{2}, w=\frac{1}{z^{n}}, h \in K .
\end{aligned}
$$


Two orbifold bundles over $\mathbb{P}(1, n)$ given by transition functions $\left(\mu_{0}, \tau_{0}\right)$ and $\left(\mu_{1}, \tau_{1}\right)$ are isomorphic if there exist smooth functions $\phi_{1}: \tilde{U}_{1} \rightarrow K$ and $\phi_{2}: U_{2} \rightarrow K$ satisfying

$$
\begin{aligned}
\phi_{1}^{-1}\left(\sigma_{n}(z)\right) \mu_{0}(z) \phi_{1}(z) & =\mu_{1}(z) \quad \forall z \in \tilde{U}_{1} \\
\phi_{2}^{-1}(w) \tau_{0}(z) \phi_{1}(z) & =\tau_{1}(z) \quad \forall z \in \tilde{U}_{1}, w \in U_{2}, w=z^{-n} .
\end{aligned}
$$

Note that the fiber over the singular point $[0] \in U_{1}$ may not be $K$, but rather $K / \mathbb{Z}_{m}$, where $m$ is the order of $\mu(0)$. We remark in the case $n=1, \mu$ can be chosen to be Id and $\tau: \mathbb{C}^{\times} \rightarrow K$ is the standard transition function.

The clutching construction for bundles above is related to the classification of principal bundles up to isomorphism. In the case without singularities, the set of principal $K$-bundles $P \rightarrow \mathbb{P}^{1}$ is in bijection with $\pi_{1}(K)$. This can be seen as follows: the deformation retract of the transition map $\mathbb{C}^{*} \rightarrow K$ is a loop $S^{1} \rightarrow K$, whose homotopy class determines the bundle. The loop $S^{1} \rightarrow K$ can be deformed to a geodesic loop $\theta \mapsto e^{\lambda \theta}$, where $\lambda \in \mathfrak{k}$ satisfies $e^{2 \pi \lambda}=$ Id. In the case of orbifold singularities, given a bundle $P \rightarrow \mathbb{P}(1, n)$, the transition maps would now produce a geodesic path $\theta \in[0,2 \pi] \mapsto e^{\lambda \theta}, \lambda \in \mathfrak{k}$ satisfies $e^{2 \pi n \lambda}=\mathrm{Id}$, see Remark 2.3(b). The isomorphism type of the bundle $P$ is determined by the homotopy class of the classifying path $\theta \mapsto e^{\lambda \theta}$, that is, deformations keeping the endpoints Id and $e^{2 \pi \lambda}$ fixed or by applying $\operatorname{Ad}_{k}$ to the path for some $k \in K$. In this way one obtains a bijection between isomorphism classes of $K$-bundles over $\mathbb{P}(1, n)$ and elements of $\exp (2 \pi i n \cdot)^{-1}(1)$, up to conjugacy.

Connections on principal bundles can be described via their restriction to trivialization associated to the clutching construction. A connection on $P \rightarrow \mathbb{P}(1, n)$ is given by connections on trivializations $\tilde{U}_{1} \times K$ and $U_{2} \times K$ that satisfy the equivalences $(9)$ :

(a) The connection $\left.A\right|_{\tilde{U}_{1}}$ satisfies

$$
\sigma_{n}^{*} A=\mu(A),
$$

where $\mu$ acts on $A$ as a gauge transformation.

(b) By the above condition, $\sigma_{n}^{*}(\tau(A))=\tau(A)$ on $\tilde{U}_{1} \backslash\{0\}$, so $\tau(A)$ descends to a connection on $\tilde{U}_{1} \backslash\{0\} / \mathbb{Z}_{n}$. We require that this descended connection is $\left.A\right|_{U_{2} \backslash\{0\}} \cdot$

We define gauge transformations in terms of the canonical atlas. A gauge transformation $k$ on $P$ consists of $\tilde{k}_{1}=\left.k\right|_{\tilde{U}_{1}}: \tilde{U}_{1} \rightarrow K$ and $k_{2}=\left.k\right|_{U_{2}}: U_{2} \rightarrow K$ satisfying the equivalences $(9)$ :

(a) $\sigma_{n}^{*} k_{1}=\mu k_{1} \mu^{-1}$ and

(b) $\left.\tau k_{1}\right|_{\tilde{U}_{1} \backslash\{0\}} \tau^{-1}$ descends to $k_{2}$.

This description shows that the set of gauge transformations on a $P \rightarrow \mathbb{P}(1, n)$ forms a group $\mathcal{K}(P)$ under composition.

Finally we describe gauged holomorphic maps on weighted projective lines. Let $P \rightarrow \mathbb{P}(1, n)$ be a principal $K$-bundle. A gauged holomorphic map $(A, u)$ from $\mathbb{P}(1, n)$ 
to $X$ consists of gauged holomorphic maps on the bundles $\tilde{U}_{1} \times K$ and $U_{2} \times K$ that satisfy the equivalence conditions (9)

(a) $\left.(A, u)\right|_{\tilde{U}_{1}}$ satisfies $\sigma_{n}^{*}(A, u)=\mu(A, u)$ (viewing $\mu$ as a gauge transformation).

(b) By the above condition, $\sigma_{n}^{*}(\tau(A, u))=\tau(A, u)$ on $\tilde{U}_{1} \backslash\{0\}$, so it descends to a gauged holomorphic map on $\tilde{U}_{1} \backslash\{0\} / \mathbb{Z}_{n}$. We require that this descended map is $\left.(A, u)\right|_{U_{2} \backslash\{0\}}$.

Holomorphic bundles on $\mathbb{P}(1, n)$ can be described in a similar way to the unitary bundles. For holomorphic bundles, the transition functions $\mu: \tilde{U}_{1} \rightarrow G$ and $\tau$ : $\tilde{U}_{1} \backslash\{0\} \rightarrow G$ are holomorphic maps. Now any holomorphic principal bundle over $\mathbb{C}$ is trivial (see $[12$, Remark 19.6]). So, the complex gauge equivalence class of a gauged holomorphic map can be specified by $u: \tilde{U}_{1} \sqcup U_{2} \rightarrow X$ and the transition functions $\tau$ and $\mu$.

2.6. Standard form near infinity. In this section we show that we can complex gauge-transform a gauged holomorphic map on a weighted projective line to a standard form near infinity. We identify the complement of the orbifold point in $\mathbb{P}(1, n)$ with $\mathbb{C}$, and let $B_{R}$ denote an open ball of radius $R$ around 0 .

Proposition 2.2. Suppose $A$ is a connection on $P \rightarrow \mathbb{P}(1, n)$. There is a complex gauge transformation $g \in \mathcal{G}(P)$, and a trivialization of $P$ over $\mathbb{C}$ so that $\left.g A\right|_{\mathbb{C}}=$ $\mathrm{D}+\lambda d \theta$ on $\mathbb{C} \backslash B_{R}$ for some $R>0, \lambda \in \mathfrak{k}$ satisfying $e^{2 \pi n \lambda}=\mathrm{Id}$. Conversely, if $A$ is a connection on $\mathbb{C}$ such that $A=\mathrm{D}+\lambda d \theta$ on $\mathbb{C} \backslash B_{R}$, then it extends to a connection on a principal bundle over $\mathbb{P}(1, n)$.

Proof. Let $A$ be a connection on a principal bundle $P \rightarrow \mathbb{P}(1, n)$. We can transform $A$ to a flat connection in a neighborhood of infinity by complex gauge transformation as follows. Choose $R_{1}>0$ and consider $B_{2 R_{1}} \subseteq \tilde{U}_{1}$. By Theorem 6.3 , there is a unique $s: B_{2 R_{1}} \rightarrow \mathfrak{k}$ with $\left.s\right|_{\partial B_{2 R_{1}}}=0$, so that $e^{i s} A$ is a flat connection. By the uniqueness of $s$ and the symmetry of $A$ (see (11)), the complex gauge transformation $e^{i s}$ is symmetric under the $\mathbb{Z}_{n}$ action, i.e. $s \circ \sigma_{n}=\operatorname{Ad}_{\mu} s$. Let $\eta: \tilde{U}_{1} \rightarrow[0,1]$ be a radially symmetric cut-off function that is 1 on $B_{R_{1}}$ and vanishes on $\tilde{U}_{1} \backslash B_{2 R_{1}}$. It is easy to see that $e^{i \eta s}$ defines a complex gauge transformation $g$ on all of $\mathbb{P}(1, n)$. The connection $g A$ is flat near infinity.

Next we do a further unitary gauge transformation so that the connection is in standard form, working over $U_{2}$. Let $R=R_{1}^{-n}$. Choose a trivialization $\left.P\right|_{U_{2}} \rightarrow$ $U_{2} \times G$ so that $g A$ is in radial gauge outside $B_{R}$, and since $g A$ is flat, $g A=\mathrm{D}+a(\theta) \mathrm{d} \theta$, for some $a: S^{1} \rightarrow \mathfrak{k}$. We now produce a gauge transformation $k: U_{2} \rightarrow K$ such that $k g A=\mathrm{D}+\lambda \mathrm{d} \theta$ for some $\lambda \in \mathfrak{k}$. Let $k_{1}:[0,2 \pi] \rightarrow K$ be the solution of

$$
\frac{k_{1}^{-1} d k_{1}}{\mathrm{~d} \theta}=a(\theta), \quad k_{1}(0)=\mathrm{Id} .
$$

The path $\theta \mapsto k_{1}(\theta)$ can be homotoped to a geodesic $\theta \mapsto e^{\lambda \theta}, \lambda \in \mathfrak{k}$. Then, $e^{\lambda \theta} k_{1}^{-1}$ is a gauge transformation on $U_{2} \backslash B_{R}$ that is homotopic to the identity and it transforms $g A$ to $\mathrm{D}+\lambda \mathrm{d} \theta$ on $U_{2} \backslash B_{R}$. By using a cut-off function, $e^{\lambda \theta} k^{-1}$ can be extended to a 
gauge transformation $k$ on all of $U_{2}$. The holonomy of $k g A$ about infinity is $e^{2 \pi \lambda}$. Since $g(A)$ has trivial holonomy for loops close to 0 in $\tilde{U}_{1}$, we have $e^{2 \pi n \lambda}=\mathrm{Id}$.

For the converse, we construct a bundle $P \rightarrow \mathbb{P}(1, n)$. Set $\mu=e^{-2 \pi \lambda}$ and $\tau=e^{n \lambda \theta}$. We are given $\left.A\right|_{U_{2}}$. A connection $\left.A\right|_{\tilde{U}_{1} \backslash\{0\}}$ can be constructed using the transition function $\tau$. The connection $\left.A\right|_{\tilde{U}_{1} \backslash\{0\}}$ is trivial on $B_{R^{-n}} \backslash\{0\} \subseteq \tilde{U}_{1} \backslash\{0\}$, so it extends smoothly to a connection on $\tilde{U}_{1}$.

Remark 2.3. (a) (Choice of orbifold singularity) Suppose $A$ is a connection on the trivial bundle $\mathbb{C} \times K$ of the form mentioned in the above proposition, i.e. $A=\mathrm{D}+\lambda \mathrm{d} \theta$ on $\mathbb{C} \backslash B_{R}$ with $e^{2 \pi \lambda n}=\mathrm{Id}$. We can extend $A$ to a connection on principal bundles not just over $\mathbb{P}(1, n)$, but also $\mathbb{P}(1, m n)$ for any positive integer $m$.

(b) (Choice of standard form) In the lemma above, the infinitesimal holonomy $\lambda$ produces the classifying path for the bundle as $\theta \mapsto e^{\lambda \theta}$. The choice of $\lambda$ is unique up to the action of $\operatorname{Ad}_{K}$. In our description of bundles over $\mathbb{P}(1, n)$, the homotopy class of this path can be recovered from the transition functions $\mu, \tau$ as the concatenation of the path $\theta \in\left[0, \frac{2 \pi}{n}\right] \mapsto \tau^{-1}(r, 0) \tau(r, \theta)$ and $t \in[-r, 0] \mapsto \mu(-t, 0)$. This is a continuous path because $\tau^{-1}(r, 0) \tau\left(r, \frac{2 \pi}{n}\right)=$ $\mu(r, 0)^{-1}$.

The next result follows easily from Proposition 2.2.

Proposition 2.4. (Standard form near infinity for gauged holomorphic maps) Let $P \rightarrow \mathbb{P}(1, n)$ be a principal $K$-bundle and let $(A, u)$ be a gauged holomorphic map from $P$ to $X$. There is a complex gauge transformation $g$ on $P$ and a trivialization of $P$ over $\mathbb{C}$ so that $g(A, u)$ satisfies the following:

(a) There is a $\lambda \in \mathfrak{k}$ so that $g A=\mathrm{D}+\lambda \mathrm{d} \theta$ on $\mathbb{C} \backslash B_{R}$ for some $R>0$. The element $\lambda$ satisfies $e^{2 \pi n \lambda}=\mathrm{Id}$.

(b) There exists $x \in X$ such that for any $\theta \in[0,2 \pi), \lim _{r \rightarrow \infty} e^{-\lambda \theta} u(r, \theta)=x$ and $e^{2 \pi \lambda} x=x$.

Conversely, any gauged holomorphic map from $\mathbb{C} \times K$ to $X$ that satisfies the above conditions for some $n$ extends to a map on $\mathbb{P}(1, n)$ for some principal bundle $P \rightarrow$ $\mathbb{P}(1, n)$.

Remark 2.5. (Topological invariants of affine vortices) The second equivariant homology class of a gauged holomorphic map on $\mathbb{P}(1, n),[(P, A, u)] \in H_{2}^{K}(X, \mathbb{Q})$ is obtained by pushing forward the rational fundamental class of the domain $[\mathbb{P}(1, n)]$ under a map $P \times_{K} X \rightarrow E K \times_{K} X$ given by a classifying map for $P$. It is a deformation invariant of $(P, A, u)$. By Theorem 3.1, one can also associate to any finite energy affine vortex $(A, u)$ a homology class $[(P, A, u)]$. The class $[(P, A, u)] \in H_{2}^{K}(X, \mathbb{Q})$ is integral if the $G$ action on $X^{\mathrm{ss}}$ is free, $n=1$. Consider the map

$$
f: H_{2}^{K}(X, \mathbb{Q}) \rightarrow H_{2}^{K}(\text { point }, \mathbb{Q})=H_{2}(B K, \mathbb{Q}) .
$$

The class $[P]:=f_{*}([(P, A, u)])$ determines the topology of the principal bundle $P \rightarrow \mathbb{P}(1, n)$. This topological information is precisely a choice of $\lambda \in \mathfrak{k}$ satisfying $e^{2 \pi n \lambda}=\mathrm{Id}$ modulo $\operatorname{Ad}_{K}$ (see Remark 2.3). 
2.7. Equivariant convexity. The notion of convexity for symplectic manifolds first arose in work by Eliashberg and Gromov [17]. Convex symplectic manifolds have similar properties to compact manifolds. This idea is extended to Hamiltonian symplectic manifolds in Cieliebak et al. [10]. An important example of equivariantly convex spaces are symplectic vector spaces with a linear group action and a proper moment map.

Definition 2.6. A Kähler manifold $(X, \omega, J)$ with a Hamiltonian $K$-action is equivariantly convex at infinity if there is a $K$-invariant proper function $f: X \rightarrow \mathbb{R}_{\geq 0}$ and a value $c_{0}$ such that if $f(x)>c_{0}$, then

$$
\left\langle\nabla_{\xi} \nabla f, \xi\right\rangle \geq 0 \quad \forall \xi \in T_{x} X, \quad d f\left(J \Phi(x)_{X}\right) \geq 0 .
$$

Here $\nabla f \in \operatorname{Vect}(X)$ is the gradient vector field of $f$ with respect to the metric $\omega(\cdot, J \cdot)$.

The above definition is equivalent to the definition in [10], where there is an additional term $\left\langle\nabla_{J \xi} \nabla f, J \xi\right\rangle$ in the left hand side of the first equation above. But, when $X$ is Kähler $\nabla_{J \xi}=J \nabla \xi$ so that term is equal to $\left\langle\nabla_{\xi} \nabla f, \xi\right\rangle$. The condition (12) implies that the image of a finite energy affine vortex with bounded image is contained in the compact set $\left\{f \leq c_{0}\right\}$ - see Proposition 11.1 in [18].

2.8. Sobolev Spaces. In this paper, most analytic proofs are carried out in Sobolev completions of the spaces described above. We set down our conventions here. Let $\Sigma$ be a compact Riemannian manifold, possibly with a smooth boundary and $E \rightarrow \Sigma$ a vector bundle. Fix a smooth covariant derivative $\nabla$ on $E$. For any $k \in \mathbb{Z}_{\geq 0}, p \in \mathbb{R}_{>1}$ and a smooth section $\sigma \in \Gamma(\Sigma, E)$, define a norm

$$
\|\sigma\|_{W^{k, p}}^{\nabla}:=\left(\sum_{i=0}^{k} \int_{\Sigma}\left|\nabla^{i} \sigma\right|^{p} \operatorname{dvol}_{\Sigma}\right)^{1 / p} .
$$

The norms for different choices of $\nabla$ are equivalent, so $\nabla$ is dropped from the notation. The space $W^{k, p}(\Sigma, E)$ is the completion of the space of smooth sections under this norm. If $\Sigma \subset \mathbb{R}^{n}$ is an open subset whose boundary $\bar{\Sigma} \backslash$ is smooth in $\mathbb{R}^{n}$, then the same definition carries over, i.e. $W^{k, p}(\Sigma, E):=W^{k, p}(\bar{\Sigma}, E)$. Using such norms one can define the Sobolev completion $\mathcal{A}^{k, p}(P)$ of the space of connections on a principal bundle, when $(k+1) p>\operatorname{dim} \Sigma$. We can also define Sobolev spaces of maps between manifolds $W^{k, p}(\Sigma, X)$ when $k p>\operatorname{dim} \Sigma$. We refer to Appendix B of the book [45] for details. We use the notation $H^{k}:=W^{k, 2}$ for any $k \in \mathbb{Z}$.

Sobolev spaces can be defined when the value of the exponent $k$ is negative. For $k \geq 0$, let $W_{0}^{k, p}(\Sigma, E)$ be the completion of the space of compactly supported smooth sections $C_{0}^{\infty}(\Sigma, E)$ under the $W^{k, p}$ norm. Also, let $p^{\prime}:=\frac{p}{p-1}$ and assume that the bundle $E$ has a Riemannian metric $g$. Then,

$$
\|\sigma\|_{W^{-k, p}}:=\sup _{\sigma^{\prime} \in W_{0}^{k, p^{\prime}}(\Sigma, E)} \int\left(\sigma, \sigma^{\prime}\right)_{g} \mathrm{dvol}_{\Sigma} .
$$


Then, $W^{-k, p}(\Sigma, E)$ is the completion of smooth sections under the dual norm of $W_{0}^{k, p^{\prime}}$. It is the dual of the space $W_{0}^{k, p^{\prime}}(\Sigma, E)$ under the $L^{2}$-pairing of sections.

The operator

$$
\mathrm{d}: W^{-k, p}(\Sigma, P(\mathfrak{k})) \rightarrow W^{-k-1, p}\left(\Omega^{1}(\Sigma, P(\mathfrak{k}))\right)
$$

is bounded because it is obtained by dualizing

$$
\mathrm{d}^{*}: W_{0}^{k+1, p}\left(\Omega^{1}(\Sigma, P(\mathfrak{k}))\right) \rightarrow W_{0}^{k, p}(\Sigma, P(\mathfrak{k})) .
$$

Combining this observation with the Sobolev multiplication Theorem (Proposition 6.2) we see that for $p>\operatorname{dim} \Sigma$, the curvature of an $L^{p}$ connection is a distributional two-form in $W^{-1, p}$. Using these weak connections, a weak version of vortices can be defined. Let $\Sigma$ be a compact Riemann surface (possibly with a smooth boundary) and $(A, u)$ lie in $\left(L^{p} \times W^{1, p}\right)(\Sigma)$ for some $p>2$. For such a pair, the vortex equation holds in a weak sense if $F_{A, u}$ vanishes in $W^{-1, p}$, i.e. for all $\xi \in W_{0}^{1, p}(\Sigma, P(\mathfrak{k}))$, we have $\int_{\Sigma}\left\langle F_{A, u}, \xi\right\rangle=0$. If $(A, u)$ is a vortex weakly, the energy of $(A, u)$ is well-defined because the curvature $F_{A}$ is equal to $-\Phi(u)$ dvol $_{\Sigma}$ which is in $C^{0}$.

\section{FROM HOLOMORPHIC MAPS TO VORTICES}

3.1. Statement of main theorem. We prove a slightly refined version of the main result Theorem 1.1 relating affine vortices to gauged holomorphic maps from orbifold lines. This requires the following analytic definition: Fix $p>2$. We call a gauged holomorphic map $(A, u)$ on $P \rightarrow \mathbb{P}(1, n)$ p-bounded if it is smooth on $\mathbb{C}$ and on $B_{\tilde{R}} \subset \tilde{U}_{1}$, which is a neighborhood of $\infty,\left.(A, u)\right|_{B_{\tilde{R}}} \in L^{p} \times W^{1, p}$. A (complex) gauge transformation on $P$ is $p$-bounded if it is smooth on $\mathbb{C}$ and in $W^{1, p}$ in a neighborhood of $\infty$. Denote by $\mathcal{G}(P)_{\text {bd }}$ the group of $p$-bounded complex gauge transformations. By the Sobolev embedding theorem, any $p$-bounded $(A, u)$ resp. $g$ is continuous and so $u(\infty)$ resp. $g(\infty)$ is well-defined. The following is the analytic version of the main result of the paper.

Theorem 3.1. Suppose $X$ is a Kähler manifold with Hamiltonian action of a compact Lie group $K$, and $X$ is either compact or equivariantly convex at infinity with a proper moment map. Let $G$ be the complexification of $K$, and suppose $G$ action on $X^{\mathrm{ss}}$ has finite stabilizers. Let $n$ be an integer such that for any $x \in X^{\mathrm{ss}},\left|G_{x}\right|$ divides $n$. Fix $2<p<2\left(1+\frac{1}{n}\right)$.

(a) Let $(A, u)$ be a gauged holomorphic map from a principal bundle $P \rightarrow \mathbb{P}(1, n)$ that satisfies $u(\infty) \in X^{\mathrm{ss}}$. There is a p-bounded complex gauge transformation $g \in \mathcal{G}(P)_{\text {bd }}$ such that $\left.g(A, u)\right|_{\mathbb{C}}$ is a smooth finite energy symplectic vortex with bounded image. Furthermore, $g$ is unique up to left multiplication by a p-bounded unitary gauge transformation.

(b) Conversely, given any finite energy symplectic vortex with bounded image, there is a unique (up to isomorphism) $K$-bundle $P \rightarrow \mathbb{P}(1, n)$ so that $(A, u)$ extends to a p-bounded gauged holomorphic map on $P$. There is a p-bounded complex gauge transformation $g \in \mathcal{G}(P)_{\text {bd }}$ so that $g(A, u)$ is smooth over 
$\mathbb{P}(1, n)$. The gauged holomorphic map $g(A, u)$ is unique up to complex gauge transformations in $\mathcal{G}(P)$.

Remark 3.2. (Relating the statements of Theorem 1.1 and Theorem 3.1) With our new notations, the statement of Theorem 1.1 can be completed as follows. The section $\sigma: \mathbb{P}(1, n) \rightarrow P_{\mathbb{C}} / K$ is smooth on $\mathbb{C} \subset \mathbb{P}(1, n)$ and in $W^{1, p}$ in a neighborhood of $\infty$.

We now explain how Theorem 1.1 follows from Theorem 3.1. Given a gauged holomorphic map $\left(P_{\mathbb{C}}, u\right)$, choose any smooth section $\sigma: \mathbb{P}(1, n) \rightarrow P_{\mathbb{C}} / K$. The choice of $\sigma$ is equivalent to the choice of a $K$-bundle $\iota: P \hookrightarrow P_{\mathbb{C}}$. The resulting unitary object $(A, u)$ is a gauged holomorphic map defined on $P$. Let $g \in \mathcal{G}(P)_{\text {bd }}$ be the complex gauge transformation produced by Part (a) of Theorem 3.1. Then, the inclusion $g \circ \iota: P \hookrightarrow P_{\mathbb{C}}$ corresponds to a section of $P_{\mathbb{C}} / K$ that satisfies the conclusions of Theorem 1.1.

In this section, we prove the first part of Theorem 3.1. The second part is proved in Section 4.

3.2. The heat flow. In this subsection, we state some relevant results about heat flow from the work of the first author [43]. Let On closed surfaces a result of Mundet [31] produces a vortex from a gauged holomorphic map satisfying a stability condition. We will use an alternative method of producing this gauge transformation using the heat flow from the work of the first author [43]. Let $c_{0}$ denote the minimal norm of the moment map on the unstable locus,

$$
c_{0}=\inf \left\{|\Phi(x)|: x \in X,\left|K_{x}\right|=\infty\right\} .
$$

By assumption 2.1, $c_{0}>0$.

Theorem 3.3. (Heat flow for gauged holomorphic maps [43, Theorem 4.4.1]) Let $\Sigma$ be a compact Riemann surface without boundary and $\left(A_{0}, u_{0}\right)$ is a gauged holomorphic map on a principal bundle $P \rightarrow \Sigma$. In addition, assume that $\left(A_{0}, u_{0}\right)$ satisfies $E\left(A_{0}, u_{0}\right) \leq c_{0}^{2} \operatorname{vol}(\Sigma)$. Then, there is a complex gauge transformation $g=e^{i \xi}$, $\xi \in W^{2, p}(\Sigma, P(\mathfrak{k}))$ (for any $p>2$ ) such that $g(A, u)$ is a vortex. Up to unitary gauge equivalence, $g(A, u)$ is the unique vortex in the complex gauge orbit of $(A, u)$.

This result is obtained by studying the gradient flow of $\left(A_{0}, u_{0}\right)$ in the space of gauged pairs on $\Sigma$ under the vortex functional

$$
(A, u) \mapsto\left\|F_{A, u}\right\|_{L^{2}(\Sigma)}^{2}, \quad F_{A, u}:=* F_{A}+\Phi(u) .
$$

The trajectory of the flow $t \mapsto\left(A_{t}, u_{t}\right)$ lies in the complex gauge orbit of $\left(A_{0}, u_{0}\right)$ and it converges to a critical point of the functional. The bound on the energy of $\left(A_{0}, u_{0}\right)$ ensures that the critical point corresponds to $F=0$, i.e. it is a vortex and that the limit is also in the complex gauge orbit of $\left(A_{0}, u_{0}\right)$. The flow has the property of decreasing energy. This is seen by the following energy identity.

Lemma 3.4. (Cieliebak et al. [11]) Let $\Sigma$ be a compact Riemann surface without boundary and $P$ a principal $K$-bundle on it. A pair $(A, u) \in \mathcal{A}(P) \times \Gamma(\Sigma, P(X))$ 
satisfies

$$
\begin{aligned}
\frac{1}{2} \int_{\Sigma}|F(A)|^{2} & +|\Phi \circ u|^{2}+\left|\mathrm{d}_{A} u\right|^{2} \operatorname{dvol}_{\Sigma} \\
& =\int_{\Sigma}\left|\bar{\partial}_{A} u\right|^{2}+\frac{1}{2}\left|* F_{A}+\Phi(u)\right|^{2} \operatorname{dvol}_{\Sigma}+\left\langle\omega_{X}-\Phi, u\right\rangle,
\end{aligned}
$$

where $\left\langle\omega_{X}-\Phi, u\right\rangle=\int_{\Sigma} u^{*} \omega-d\langle\Phi(u), A\rangle$.

The pairing $\left\langle\omega_{X}-\Phi, u\right\rangle$ is a topological invariant of $(A, u)$ and is preserved by the flow. Since $\left\|F_{\left(A_{t}, u_{t}\right)}\right\|_{L^{2}}^{2}$ decreases with time $t$, the same is the case with the energy $E\left(A_{t}, u_{t}\right)$.

We recall several other results about gauged holomorphic maps on Riemann surfaces with boundary needed for the proof of Theorem 3.1 (a). The first one says that any gauged holomorphic map which is an approximate solution to the vortex equations may be complex gauge transformed into one.

Proposition 3.5. ([43, Proposition 4.3.1]) Let $p>2$. Suppose $\Sigma$ is a compact connected Riemann surface with a smooth boundary. Let $\left(A_{i}, u_{i}\right) \in \mathcal{H}(P, X)_{L^{p} \times W^{1, p}}$ be a sequence and $\left(A_{\infty}, u_{\infty}\right) \in \mathcal{H}(P, X)_{L^{p} \times W^{1, p}}$ be such that $A_{i} \rightarrow A_{\infty}$ in $L^{p}$ and there is a finite set $Z \subseteq \Sigma$ so that $u_{i} \rightarrow u_{\infty}$ in $C^{0}$ on compact subsets of $\Sigma \backslash(Z \cup \partial \Sigma)$. Also, $F_{i}:=* F\left(A_{i}\right)+u_{i}^{*} \Phi \rightarrow 0$ in $W^{-1, p}$. Then, there exist constants $C$ and $i_{0}$ so that for $i>i_{0}$, there is a complex gauge transformation $\exp i \xi_{i}, \xi_{i} \in W_{0}^{1, p}(\Sigma, P(\mathfrak{k}))$ so that $\left(\exp i \xi_{i}\right)\left(A_{i}, u_{i}\right)$ is a vortex and satisfies $\left\|\xi_{i}\right\|_{W^{1, p}}<8 C\left\|F_{i}\right\|_{W^{-1, p}}$.

The next result we will need is Proposition 4.3.3 in [43]. Roughly it says that in a complex gauge orbit, there is at most one vortex up to gauge. The proof is reproduced, because it will be useful in understanding the corresponding result for affine vortices.

Proposition 3.6. Let $p>2$ and $\Sigma$ be a compact connected Riemann surface with a smooth boundary. Let $\left(A_{0}, u_{0}\right),\left(A_{1}, u_{1}\right) \in L^{p} \times W^{1, p}$ be vortices on a principal bundle $P \rightarrow \Sigma$ that are related by a complex gauge transformation $g \in \mathcal{G}^{1, p}(P)$, i.e. $\left(A_{1}, u_{1}\right)=g\left(A_{0}, u_{0}\right)$ and assume $g(\partial \Sigma) \subseteq K$. Then, $\left(A_{0}, u_{0}\right)$ and $\left(A_{1}, u_{1}\right)$ are gauge-equivalent, i.e. $g \in \mathcal{K}^{1, p}(P)$.

Proof. After a gauge transformation, we can assume $\left(A_{1}, u_{1}\right)=e^{i \xi}\left(A_{0}, u_{0}\right)$, where $\xi \in \Gamma(\Sigma, P(\mathfrak{k}))_{1, p}$ and $\left.\xi\right|_{\partial \Sigma}=0$. Let $\left(A_{t}, u_{t}\right):=e^{i t \xi}\left(A_{0}, u_{0}\right)$. We know $F_{A_{0}, u_{0}}=$ $F_{A_{1}, u_{1}}=0$ weakly. For $\left.\xi\right|_{\partial \Sigma}=0$,

$$
\begin{aligned}
\frac{d}{d t} \int_{\Sigma}\left\langle * F_{A_{t}, u_{t}}, \xi\right\rangle & =\int_{\Sigma}\left\langle\mathrm{d}_{A_{t}}^{*} \mathrm{~d}_{A_{t}} \xi+u_{t}^{*} d \Phi\left(J(\xi)_{u_{t}}\right), \xi\right\rangle_{\mathfrak{k}} \\
& =\left\|\mathrm{d}_{A_{t}} \xi\right\|_{L^{2}}^{2}+\int_{\Sigma} \omega_{u_{t}}\left((\xi)_{u_{t}}, J(\xi)_{u_{t}}\right) \geq 0 .
\end{aligned}
$$

The inequality is strict for non-zero $\xi$. So, $\xi=0$ and $\left(A_{0}, u_{0}\right)$ and $\left(A_{1}, u_{1}\right)$ are gauge-equivalent. 
3.3. Elliptic regularity for gauged holomorphic maps. This section contains some results related to elliptic regularity for vortices and gauged holomorphic maps required in the proof of Theorem 3.1 (a). Most of these lemmas are similar to results already present in the literature. We include them here, because we require extensions to slightly lower regularity spaces.

Lemma 3.7. (Gromov convergence for vortices [34], [51]) Let $p>2$ and $\Sigma_{i}$ be a sequence of precompact sets exhausting a Riemann surface $\Sigma$,

$$
\Sigma_{1} \Subset \Sigma_{2} \Subset \cdots \Subset \Sigma, \quad \bigcup_{i} \Sigma_{i}=\Sigma .
$$

Suppose for each $i,\left(A_{i}, u_{i}\right)$ is a smooth vortex on $\Sigma_{i}$,

$$
\sup _{i} E\left(\Sigma_{i},\left(A_{i}, u_{i}\right)\right)<\infty
$$

and there is a compact set $S \subset X$ containing the images of all the $u_{i}$. Let $Z$ denote the set of points $z$ for which there is a sequence $z_{i} \rightarrow z$ in $\Sigma$ such that $\left|\mathrm{d}_{A_{i}} u_{i}\left(z_{i}\right)\right| \rightarrow \infty$ as $i \rightarrow \infty$. Then, the set $Z$ is finite and after passing to a subsequence, there are gauge transformations $k_{i} \in H^{2}\left(\Sigma_{i}, K\right)$ and a finite energy vortex $\left(A_{\infty}, u_{\infty}\right) \in$ $H_{\text {loc }}^{1} \times H_{\text {loc }}^{2}$ over $\mathbb{C}$ such that

(a) $k_{i} A_{i} \rightarrow A_{\infty}$ in $H^{1}$, and strongly in $L^{p}$, on compact subsets of $\Sigma$.

(b) $u_{i} \rightarrow u_{\infty}$ in $H^{2}$, and strongly in $W^{1, p}$ and $C^{0}$, on compact subsets of $\Sigma \backslash Z$.

Proof. This lemma is a combination of results in [51] and [34]. We provide an outline of the proof. The bounded energy condition implies a curvature bound

$$
\left\|F\left(A_{i}\right)\right\|_{L^{2}\left(\Sigma_{i}\right)}<c
$$

for all $i$. By Uhlenbeck's theorem for non-compact domains (Theorem A' in [45]), after passing to a subsequence, there are gauge transformations $k_{i} \in H^{2}\left(\Sigma_{i}\right)$ and a limit connection $A_{\infty}$ on the trivial bundle $\Sigma \times K$ such that $k_{i} A_{i} \rightarrow A_{\infty}$ in $H^{1}$ on compact subsets of $\Sigma$.

We first prove convergence on compact sets for bounded first derivatives. On a compact set $Q \subset \Sigma$, if there is a bound on $\left\|\mathrm{d}_{A_{i}} u_{i}\right\|_{L^{p}(Q)}$, then a subsequence of $k_{i} u_{i}$ converges weakly in $H^{2}(Q)$. To see this, write $k_{i} A_{i}=\mathrm{D}+a_{i}$. Since all the $u_{i}$ map to a compact set and $a_{i}$ is bounded in $L^{p},\left(a_{i}\right)_{u_{i}}$ is also bounded in $L^{p}$, hence there is an $L^{p}(Q)$ bound on $d\left(k_{i} u_{i}\right)$. This implies $k_{i} u_{i}$ converges weakly in $W^{1, p}(Q)$ and strongly in $C^{0}$ to a limit $u_{\infty}$ (see Theorem B.4.2 in [29]). By Lemma 3.8 below, after passing to a subsequence $k_{i} u_{i} \rightarrow u_{\infty}$ in $H^{2}(Q)$. A standard diagonalization argument shows that there is a subsequence that works for all compact subsets $Q$.

In the absence of a first derivative bound, one has bubbling. As in Ott [34], there is a finite set $Z \subset \Sigma$ where bubbling occurs. That is, after passing to a subsequence, $\left\|\mathrm{d}_{A_{i}} u_{i}\right\|_{L^{p}(Q)}$ is bounded on compact subsets $Q \subset \Sigma \backslash Z$. There is a map $u_{\infty}: \Sigma \backslash Z \rightarrow X$ such that $k_{i} u_{i}$ converges weakly to $u_{\infty}$ in $H^{2}(Q)$ for all compact subsets $Q \subset \Sigma \backslash Z$. The map $u_{\infty}$ extends to a continuous map on $\Sigma$. Since we are in the Kähler case, this has a much easier proof than that in Ott [34]: Choose $z_{0} \in Z$ and a small neighborhood $U \subset \Sigma$ so that $U \cap Z=\left\{z_{0}\right\}$. By Lemma 4.3, 
there is a complex gauge transformation $g \in H^{2}(U) \hookrightarrow C^{0}(U)$ such that $g A_{\infty}$ is the trivial connection. Complex gauge transformations preserve holomorphicity, so $\bar{\partial}\left(g u_{\infty}\right)=0$ on $U \backslash\left\{z_{0}\right\}, g u_{\infty}$ is smooth on $U \backslash\left\{z_{0}\right\}$ and the image $g u_{\infty}\left(U \backslash\left\{z_{0}\right\}\right)$ is contained in a compact set. By the removable singularity theorem of complex analysis ([39, Theorem 3.1]), we have $g u_{\infty}$ extends smoothly over $z_{0}$. Therefore $u_{\infty}$ extends over $z_{0}$ as an $H^{2}$ map, and so $u_{\infty} \in H_{\text {loc }}^{2}$.

The following elliptic regularity result (Theorem 3.1 in Cieliebak et al.[10]) is standard, but we provide a self-contained proof.

Lemma 3.8. (Elliptic regularity for gauged holomorphic maps) Let $s \geq 1$ be an integer, $p>2, \Sigma \subseteq \mathbb{C}$ be a pre-compact open set with smooth boundary and $\left(A_{i}, u_{i}\right)$ be a sequence of gauged holomorphic maps on $\Sigma$ such that $A_{i} \rightarrow A_{\infty}$ in $H^{s}(\Sigma)$ and $u_{i} \rightarrow u_{\infty}$ in $W^{1, p}(\Sigma)$, then $u_{i} \rightarrow u_{\infty}$ in $H^{s+1}\left(\Sigma^{\prime}\right)$, for any compact set $\Sigma^{\prime}$ that is contained in $\operatorname{int}(\Sigma)$.

Proof. The proof is by induction on $s$. We first assume the result for some $s>1$ and prove it for $s+1$. Note that it suffices to work locally in $X$ : Choose an atlas $X=\cup_{\alpha} \mathcal{V}_{\alpha}$ such that $\mathcal{V}_{\alpha}$ is bi-holomorphic to an open subset of $\mathbb{C}^{N}$. Since $u_{i} \rightarrow u_{\infty}$ in $C^{0}$, we can find a finite cover $\Sigma=\cup_{\beta} \mathcal{U}_{\beta}$ such that for $u_{i}\left(\mathcal{U}_{\beta}\right)$ is contained in a single $\mathcal{V}_{\alpha}$ for large $i$. So, now we can think of $u_{i}$ as mapping to $\mathbb{C}^{N}$.

In each chart we apply a combination of Sobolev multiplication and regularity theorems. As in [10], write $A_{i}=\mathrm{D}+\Theta_{i} d x+\Psi_{i} d y$, where $\Psi_{i}, \Theta_{i} \in H^{s}(\Sigma, \mathfrak{k})$ and the holomorphicity equation for $\left(A_{i}, u_{i}\right)$ is

$$
-\bar{\partial} u_{i}=\left(\Theta_{i}\right)_{u_{i}}+J_{X}\left(\Psi_{i}\right)_{u_{i}} .
$$

See (5) for the notation $\left(\Theta_{i}\right)_{u_{i}}$. We know that both $A_{i}$ and $u_{i}$ weakly converge in $H^{s}$, so $\Psi_{i}, \Theta_{i}$ and $u_{i}$ are uniformly bounded in $H^{s}$. We next show that $\left(\Theta_{i}\right)_{u_{i}}$ and $\left(\Psi_{i}\right)_{u_{i}}$ are uniformly bounded in $H^{s}$. For this, define an operator $L_{x}$ for every $x \in X$,

$$
L_{x}: \mathfrak{k} \rightarrow T_{x} X, \quad \xi \mapsto \xi_{X}(x) .
$$

The function $L: X \rightarrow \operatorname{Hom}(\mathfrak{k}, T X) \simeq \operatorname{Hom}\left(\mathfrak{k}, \mathbb{C}^{N}\right)$ given by $x \mapsto L_{x}$ is smooth. So, given $u: \Sigma \rightarrow X$, we obtain a section

$$
L(u):=L \circ u \in \Gamma\left(\Sigma, \operatorname{Hom}\left(\mathfrak{k}, u^{*} T X\right)\right) \simeq \Gamma\left(\Sigma, \operatorname{Hom}\left(\mathfrak{k}, \mathbb{C}^{N}\right)\right) .
$$

The term $\left(\Theta_{i}\right)_{u_{i}}$ can be seen as a product $\left(\Theta_{i}\right)_{u_{i}}=L\left(u_{i}\right) \Theta_{i}$. In the following discussion, the constant $c$ indicates a constant that is independent of $i$, whose value varies across appearances. Since $L$ is smooth, $\left\|L\left(u_{i}\right)\right\|_{H^{s}\left(\mathcal{U}_{\alpha}\right)}<c$ for all $i, \alpha$. By Sobolev multiplication (Proposition 6.2), for $s>1$,

$$
\left\|L\left(u_{i}\right) \Theta_{i}\right\|_{H^{s}\left(\mathcal{U}_{\alpha}\right)} \leq c\left\|L\left(u_{i}\right)\right\|_{H^{s}\left(\mathcal{U}_{\alpha}\right)}\left\|\Theta_{i}\right\|_{H^{s}\left(\mathcal{U}_{\alpha}\right)} .
$$

By holomorphicity $(15),\left\|\bar{\partial} u_{i}\right\|_{H^{s}\left(\mathcal{U}_{\alpha}\right)}<c$ for all $i, \alpha$. By elliptic regularity for curves in $\mathbb{C}^{N}$,

$$
\left\|u_{i}\right\|_{H^{s+1}\left(\mathcal{U}_{\alpha}^{\prime}\right)} \leq c\left(\left\|\bar{\partial} u_{i}\right\|_{H^{s}\left(\mathcal{U}_{\alpha}\right)}+\left\|u_{i}\right\|_{L^{2}\left(\mathcal{U}_{\alpha}\right)}\right)
$$


where $\overline{\mathcal{U}}_{\alpha}^{\prime} \subseteq \mathcal{U}_{\alpha}$ and $c$ depends on $\mathcal{U}_{\alpha}, \mathcal{U}_{\alpha}^{\prime}$. By choosing $\mathcal{U}_{\alpha}^{\prime}$ such that they cover $\Sigma^{\prime}$, we obtain a uniform bound on $\left\|u_{i}\right\|_{H^{s+1}\left(\Sigma^{\prime}\right)}$ and so, after passing to a subsequence, $u_{i} \rightarrow u_{\infty}$ in $H^{s+1}\left(\Sigma^{\prime}\right)$.

It remains to prove the result for $s=1$. The Sobolev multiplication step (17) is not applicable for $s=1$. Instead, we now have a bound $\left\|L\left(u_{i}\right)\right\|_{W^{1, p}\left(\mathcal{U}_{\alpha}\right)}<c$ and then by the Sobolev multiplication theorem (Proposition 6.2),

$$
\left\|L\left(u_{i}\right) \Theta_{i}\right\|_{H^{1}\left(\mathcal{U}_{\alpha}\right)} \leq c\left\|L\left(u_{i}\right)\right\|_{W^{1, p}\left(\mathcal{U}_{\alpha}\right)}\left\|\Theta_{i}\right\|_{H^{1}\left(\mathcal{U}_{\alpha}\right)} .
$$

The rest of the proof of Lemma 3.8 is same as the $s>1$ case.

Lemma 3.9. (Regularity for vortices) Let $p>2$. Given a finite energy vortex $(A, u) \in\left(L_{\text {loc }}^{p} \times C^{0}\right)(\mathbb{C})$ on the trivial bundle $\mathbb{C} \times K$, there is a gauge transformation $k \in W_{\text {loc }}^{1, p}(\mathbb{C})$ such that $k(A, u)$ is smooth on $\mathbb{C}$.

Proof. We first carry out the proof for the closure $\Sigma$ of a bounded open subset with smooth boundary in $\mathbb{C}$. The proof is by induction. We first deal with the case when $(A, u)$ has high enough regularity. We assume $(A, u) \in\left(W^{m, p} \times W^{m+1, p}\right)(\Sigma)$, where $m p>2$. The idea of the proof is to find a smooth reference connection $A_{0}$ and put $A$ is Coulomb gauge with respect to $A_{0}$ by a gauge transformation $k \in W^{m+1, p}$. Writing $k A=A_{0}+\alpha$, we have $\mathrm{d}_{A_{0}}^{*} \alpha=0$. Since $u \in W^{m+1, p}(\Sigma), \Phi(k u)$ is also in the same class and by the vortex equation $F_{k A} \in W^{m+1, p}(\Sigma) \hookrightarrow W^{m, p}(\Sigma)$. Writing

$$
\mathrm{d}_{A_{0}} \alpha=F_{k A}-\frac{1}{2}[\alpha \wedge \alpha] \Longrightarrow \mathrm{d}_{A_{0}} \alpha \in W^{m, p} \text {. }
$$

So, we have $\alpha \in W_{\text {loc }}^{m+1, p}(\Sigma \backslash \partial \Sigma)$ and so, $k A \in W_{\text {loc }}^{m+1, p}(\Sigma \backslash \partial \Sigma)$. By elliptic regularity (similar to proof of Lemma 3.8, see (15)), we have $W_{\text {loc }}^{m+2, p}$ control over $k u$ on $\Sigma \backslash \partial \Sigma$. This procedure can be applied inductively to gauge transform $(A, u)$ to a smooth vortex on $\Sigma \backslash \partial \Sigma$.

Now, we discuss the case when $(A, u)$ has lower regularity than what is required in the previous paragraph, i.e. when $A \in L_{\text {loc }}^{p}$. As earlier, it is possible to gauge transform $A$ to a connection in Coulomb gauge (see [45, Theorem 8.3]) with respect to a nearby connection $A_{0}$, i.e. there exists $k \in W^{1, p}(\Sigma, K)$ such that $k A=A_{0}+\alpha$ and $\mathrm{d}_{A_{0}}^{*} \alpha=0$. However, the next step (18) requires that $m p>2$, which fails when $(A, u) \in L_{\mathrm{loc}}^{p} \times C^{0}$. The regularity of $\alpha$ is brought up by a bootstrapping procedure using the equation in (18). First assume $2<p<4$. Set $q_{0}:=p$. There exists $m$ and a sequence $2<q_{0}<\cdots<q_{m-1} \leq 4, q_{m}>4$ such that

$$
q_{0}=p, \quad q_{i}<\frac{2 q_{i-1}}{4-q_{i-1}} \quad i \geq 1 .
$$

Suppose $\alpha \in L^{q_{i}}$. Then, by Hölder's inequality $[\alpha \wedge \alpha] \in L^{q_{i} / 2}$. Since $k u$ is in $C^{0}$, the term $\Phi(k u)$ is also in $C^{0}$, and by the vortex equation $F_{k A}$ is in $C^{0}$, hence also in $L^{q_{i} / 2}$. Bootstrapping using (18), we get $\alpha \in W^{1, q_{i} / 2} \hookrightarrow L^{q_{i+1}}$. By repeating this procedure, we end up with $\alpha \in L^{q_{m}}$. If $p>4$, then $m$ is equal to 0 in the above discussion, and we straightaway have $\alpha \in L^{q_{m}}$. Using (18) one more time, we get $\alpha \in W^{1, q_{m} / 2}$. 
By elliptic regularity (similar to proof of Lemma 3.8, see (15)), $u \in W_{\mathrm{loc}}^{2, q_{m} / 2}(\Sigma \backslash \partial \Sigma)$. Now, the inductive step of the previous paragraph is applicable.

The proof extends to all of the complex line via an exhaustion argument carried out in Proposition 77 in [51], which carries over to this case with lower regularity.

3.4. Proof of Theorem 3.1(a). We now present the proof of the main result Theorem 3.1(a) modulo some technical results which are deferred to the following subsections.

Proof. (Proof of Theorem 3.1 (a)) Starting from a stable gauged holomorphic map, we first produce a sequence of vortices on exhausting subsets of the complex line, which has a limit modulo bubbling that is a finite energy affine vortex. Given a stable gauged holomorphic map $(A, u)$ defined on a principal bundle $P$ over $\mathbb{P}(1, n)$ (resp. $\mathbb{P}^{1}$ ), we may assume that $u(\infty) \in \Phi^{-1}(0)$. Since $u(\infty) \in X^{\mathrm{ss}}$, this condition can be achieved by a complex gauge transformation. This preliminary step is to simplify arguments later in the proof. We now apply Proposition 3.10 below to obtain a sequence of vortices $\left(A_{i}, u_{i}\right)$ defined on the annuli $A\left(1 / R_{i}, R_{i}\right)$ (resp. balls $\left.B_{R_{i}}\right)$ that satisfy $\left(A_{i}, u_{i}\right)=g_{i}(A, u)$ where $g_{i} \in H^{2}\left(B_{R_{i}}\right)$ is a complex gauge transformation. The sequence converges in the sense of Proposition 3.10 to a finite energy affine vortex $\left(A_{\infty}, u_{\infty}\right)$. We remark that from the output of Proposition 3.10, we replace $g_{i} k_{i}$ by $g_{i}$ and drop $k_{i}$ from our notation in this proof.

In the remainder of the proof, we will show that the limit vortex is complex gauge equivalent to every vortex in the sequence. We first construct the necessary complex gauge transformation outside a large ball. Since $\left(A_{\infty}, u_{\infty}\right)$ has finite energy and bounded image, by Proposition $4.2, \Phi\left(u_{\infty}(z)\right) \rightarrow 0$ as $z \rightarrow \infty$. So we can choose an $R>0$ such that $u_{\infty}\left(\mathbb{C} \backslash B_{R}\right) \subseteq X^{\mathrm{ss}}$ and $Z \subseteq B_{R}$. After possibly passing to a subsequence, we have $u_{i}\left(\mathbb{C} \backslash B_{R}\right) \subseteq X^{\text {ss }}$. Using Lemma 3.16, after passing to a subsequence again, $g_{i}$ has a limit $g_{\infty} \in W_{\text {loc }}^{1, p}$. In particular, we have

$$
g_{i} \rightarrow g_{\infty} \quad \text { in } W^{1, p}(Q, G) \text { for all compact subsets } Q \subset \mathbb{C} \backslash B_{R} .
$$

We remark that in the case that the $G$-action on $X^{\text {ss }}$ is free, the proof of this statement is more straightforward and does not require Lemma 3.16: Observe that by Assumption 2.1, semistable orbits are relatively closed in $X^{\mathrm{ss}}$. This implies that for all $x \in \mathbb{C} \backslash B_{R}$, the points $u_{i}(x)$ and $u_{\infty}(x)$ are in the same $G$-orbit. By the free action, there is a unique $g_{\infty}$ as above. Further the $W^{1, p}$ convergence of the maps $u_{i}$ in compact subsets of $\mathbb{C} \backslash B_{R}$ implies a similar convergence of the sequence $g_{i}$ to $g_{\infty}$. By Lemma 3.17, we have

$$
\left(A_{\infty}, u_{\infty}\right)=g_{\infty}(A, u) \quad \text { on } \mathbb{C} \backslash B_{R} .
$$

The limit gauge transformation produced outside the ball extends to inside the ball. By Proposition 3.15, the gauge transformation $g_{\infty}$ extends to all of $\mathbb{C}$ while satisfying $\left(A_{\infty}, u_{\infty}\right)=g_{\infty}(A, u)$ on $\mathbb{C}$. The complex gauge transformation $g_{\infty}$ is in $W_{\text {loc }}^{1, p}(\mathbb{C})$. 
We emphasize that the above argument shows there is no bubbling, i.e. $Z=\emptyset$ and in the orbifold case 0 is not a singular point. By the conclusion of Proposition 3.15 , the sequence $\left(A_{i}, u_{i}\right)$ which is equal to $g_{i}(A, u)$ converges to $g_{\infty}(A, u)$ weakly in $\left(L^{p} \times W^{1, p}\right)\left(B_{R}\right)$, and this limit is same as $\left(A_{\infty}, u_{\infty}\right)$. So, the quantity $\left\|\mathrm{d}_{A_{i}} u_{i}\right\|_{L^{p}\left(B_{R}\right)}$, which is gauge invariant, is uniformly bounded for all $i$. By Proposition 78 in [51], modulo gauge transformations, the sequence $\left(A_{i}, u_{i}\right)$ converges smoothly to a limit vortex on compact subsets of $\operatorname{int}\left(B_{R}\right)$. This implies that $\left|d_{A_{i}} u_{i}\right|_{L^{\infty}}$ is uniformly bounded on a small compact neighborhood of $Z$, which contradicts the characterization of $Z$ in Proposition 3.10. So there is no bubbling, that is, the bubbling set $Z$ is empty.

We next show that the complex gauge transformation $g_{\infty}$ needed to make the pair into a vortex has the claimed regularity, that is, $g_{\infty}$ is $p$-bounded on the bundle $P$ over the weighted projective line. Using Lemma 3.9 , we can modify $g_{\infty}$ by a gauge transformation in $W_{\text {loc }}^{1, p}$, so that $g_{\infty}(A, u)$ is smooth. By applying Lemma 3.18 in neighborhoods in $\mathbb{C}$, we conclude that $g_{\infty}: \mathbb{C} \rightarrow G$ is smooth. Write $g_{\infty}=k_{\infty} e^{i \xi_{\infty}}$. In the beginning of the proof, we assumed $\Phi(u(\infty))=0$. We also have $\lim _{r \rightarrow \infty} u_{\infty}(r, \theta) \in \Phi^{-1}(0)$ for any $\theta$. This implies that $\xi_{\infty}(r, \theta) \rightarrow 0$ as $r \rightarrow \infty$. The complex gauge transformation $e^{i \xi_{\infty}}$ on $P$ is smooth on $\mathbb{C}$ and continuous at $\infty$. We now show that $e^{i \xi_{\infty}} \in \mathcal{G}(P)_{\text {bd }}$. Given the finite energy vortex $e^{i \xi_{\infty}}(A, u)$, Theorem 3.1(b) shows that the vortex extends to a gauged holomorphic map over a principal $K$-bundle $P^{\prime} \rightarrow \mathbb{P}(1, n)$. (The proof of part (b) of the theorem is independent of part (a)). The bundles $P$ and $P^{\prime}$ are isomorphic by the following argument. The limit $u(\infty, \theta):=\lim _{r \rightarrow \infty} u(r, \theta)$ is well-defined and satisfies $u(\infty, \theta)=k(\theta) u(\infty, 0)$ for a path $k:[0,2 \pi] \rightarrow K$, where $k(0)=\operatorname{Id}$ and $k(2 \pi)^{n}=\mathrm{Id}$. By Remark 2.3, the bundle $P$ is uniquely determined by the path $k$. The bundle $P^{\prime}$ is analogously determined by a path given by $e^{i \xi_{\infty}} u$, this path is same as $k$. Since $e^{i \xi_{\infty}}(A, u)$ is a $p$-bounded gauged holomorphic map on $P, e^{i \xi_{\infty}} u$ is in $W^{1, p}$ on a neighborhood of $\infty$ in $\tilde{U}_{1}$, and so the same is true of $\xi_{\infty}$, therefore $e^{i \xi_{\infty}} \in \mathcal{G}(P)_{\mathrm{bd}}$.

To finish the proof Theorem 3.1 (a), it remains to show that the vortex $e^{i \xi_{\infty}}(A, u)$ is unique up to gauge transformations. This is proved separately in Proposition 4.5 using Theorem 3.1 (b).

3.5. Producing a limit modulo bubbling. The main result of this section is Proposition 3.10, which can be described informally as follows. We choose a sequence of balls, or annuli in the orbifold case, that exhaust the complex line. Given a stable gauged holomorphic map on the weighted projective space, there exists a sequence of gauge transformations that transform the gauged holomorphic map to a sequence of vortices on these increasing balls (resp. annuli). This sequence of vortices converges modulo bubbling to a limit vortex defined on all of the complex line.

Proposition 3.10. (A limit modulo bubbling) Suppose $(A, u)$ is a stable gauged holomorphic map defined on a principal $K$-bunde $P \rightarrow \mathbb{P}(1, n)$. Let $p>2$ and $R_{i} \rightarrow \infty$ be an increasing sequence, with $R_{1} \geq 2$. We denote by $A\left(1 / R_{i}, R_{i}\right) \subset \mathbb{C}$ an annulus of radii $1 / R_{i}$ and $R_{i}$. 
(a) There exists a sequence of complex gauge transformations $g_{i} \in H^{2}\left(B_{R_{i}}, G\right)$ such that the restriction of $\left(A_{i}, u_{i}\right):=g_{i}(A, u)$ to the annulus $A\left(1 / R_{i}, R_{i}\right)$ is a vortex whose energy is uniformly bounded. The curvature $\left\|F_{g_{i} A}\right\|_{L^{2}\left(B_{1 / R_{i}}\right)}$ is also uniformly bounded.

(b) Let $Z$ be the set of points $z \in \mathbb{C} \backslash\{0\}$ for which there is a sequence $z_{i} \rightarrow z$ such that $\left|\mathrm{d}_{A_{i}} u_{i}\left(z_{i}\right)\right| \rightarrow \infty$ as $i \rightarrow \infty$. Then, the set $Z$ is finite and there exists a sequence of gauge transformations $k_{i} \in H^{2}\left(B_{R_{i}}, K\right)$ and a vortex $\left(A_{\infty}, u_{\infty}\right) \in\left(H_{\text {loc }}^{1} \times H_{\text {loc }}^{2}\right)(\mathbb{C})$ such that

(1) $k_{i} A_{i}$ converges to $A_{\infty}$ weakly in $H^{1}$, and strongly in $L^{p}$ on compact subsets of $\mathbb{C}$.

(2) $k_{i} u_{i}$ converges to $u_{\infty}$ weakly in $H^{2}$ and strongly in $W^{1, p}$ on compact subsets of $\mathbb{C} \backslash(Z \cup\{0\})$.

The vortex $\left(A_{\infty}, u_{\infty}\right)$ has finite energy and bounded image.

We outline the proof of Proposition 3.10, starting with the manifold case. In this case, the stable gauged holomorphic map is defined on the projective line. We use a sequence of metrics that interpolate between the Euclidean metric on the affine line and the Fubini-Study metric. In particular, the sequence of metrics is constructed so that the sequence of increasing balls in the Proposition respectively have the Euclidean metric on them. With respect to each metric in the sequence, one can apply the heat flow result Theorem 3.3 to the given gauged holomorphic map. For most (all but finite) metrics in the sequence, the limit of the heat flow is a vortex under the respective metric. The sequence of vortices, when respectively restricted to the sequence of increasing balls, satisfies the necessary energy bound, and hence we obtain the convergence in the sense of part (b) of Proposition 3.10.

We remark that in the case that the git quotient $X / / G$ is a manifold, a slightly weaker result suffices for the proof of Theorem 3.1: The elements of the sequence $\left(A_{i}, u_{i}\right)$ can be taken to be vortices on the balls $B_{R_{i}}$, instead of annuli. The set $Z$ of bubbling points would then be a subset of $\mathbb{C}$, not of $\mathbb{C} \backslash\{0\}$. The additional curvature bound $\left\|F_{g_{i} A}\right\|_{L^{2}\left(B_{1 / R_{i}}\right.}$ would not be required. In part (b), the convergence of the sequence $k_{i} u_{i}$ would be in compact subsets of $\mathbb{C} \backslash Z$.

In the orbifold case, we start with a stable gauged holomorphic map defined on the weighted projective line. The proof differs in this case because the heat flow does not apply when the base space is an orbifold. To get around this issue we work on a cover of the weighted projective line that is ramified at the origin. The cover is bi-holomorphic to the projective line. Unfortunately, the lift of the Euclidean metric to this cover degenerates at the ramification point. We construct a sequence of metrics by interpolating between the Fubini-Study metric near infinity, a lift of the Euclidean metric on a sequence of increasing annuli and the Euclidean metric scaled by a factor near the origin. Repeating the heat flow process produces a sequence of vortices on the increasing annuli. The sequence converges in the sense of the Proposition on the complex line punctured at the origin. Additional technical details are required to remove the singularity at the origin for the limit vortex. 


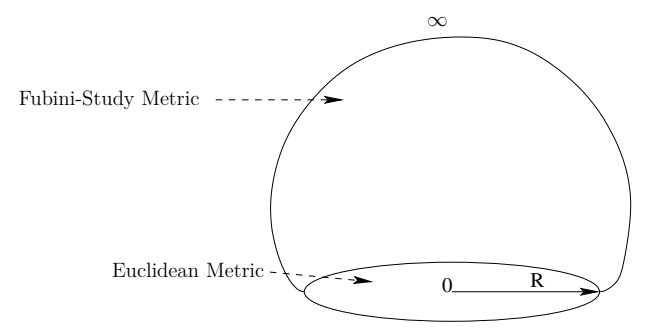

Figure 1. The metric $\operatorname{dvol}_{R}$ on $\mathbb{P}^{1}$

Definition 3.11. (Ramified cover of the orbifold line) We denote by $\tilde{\mathbb{P}}$ an $n$-cover of $\mathbb{P}(1, n)$ that is ramified at 0 . The cover $\tilde{\mathbb{P}}$ is bi-holomorphic to the projective line $\mathbb{P}^{1}$ and the covering map is

$$
\pi: \tilde{\mathbb{P}} \rightarrow \mathbb{P}(1, n) \quad[x: y] \mapsto\left[x^{n}: y\right] .
$$

To see that this map is well-defined, recall that $\mathbb{P}(1, n)$ can be defined as

$$
\mathbb{P}(1, n):=\left(\mathbb{C}^{2} \backslash\{0\}\right) / \sim, \quad(x, y) \sim\left(\lambda^{n} x, \lambda y\right) \forall \lambda \in \mathbb{C}^{\times} .
$$

It is useful to have chart-based description of $\tilde{\mathbb{P}}$. Recall $\mathbb{P}(1, n)$ is constructed using two charts $\tilde{U}_{1}$ and $U_{2}$ (see $\left.(8)\right)$. The cover $\tilde{\mathbb{P}}$ is made up of charts $\tilde{U}_{1}, \tilde{U}_{2}$. The projection $\pi: \tilde{U}_{2} \rightarrow U_{2}:=w \mapsto w^{n}$ is an $n$-cover ramified at 0 . We consider $\pi^{*}(A, u)$ and perform all the following steps maintaining symmetry under the $\mathbb{Z}_{n}$-action.

Next, we describe a family of metrics on the cover of the weighted projective line that agrees with a lift of the Euclidean metric on a sequence of exhausting subsets. The Euclidean area form on $\mathbb{C} \subset \mathbb{P}(1, n)$ pulls back to an area form

$$
\mathrm{dvol}_{\text {orb }}=n^{2}\left(|x|^{2}+|y|^{2}\right)^{n-1} d x \wedge d y
$$

on $\mathbb{C} \subset \tilde{\mathbb{P}}$. In case $n>1$, the corresponding metric degenerates at the origin. We define a metric $\operatorname{dvol}_{R}$ that is dependent on a parameter $R>0$ (see (21)) and is equal to dvol orb on the annulus $A(1 / R, R)$ of radii $1 / R$ and $R$, it is equal to the FubiniStudy metric in a neighborhood of $\infty$ and a Euclidean metric in a neighborhood of 0 . The modification near the origin is to remove the degeneracy of the metric.

Definition 3.12. (A family of metrics on the orbifold line) Let $\eta: \mathbb{C} \rightarrow[0,1]$ be a radially symmetric cut-off function that is 1 in the unit ball $B_{1}$ and 0 on $\mathbb{C} \backslash B_{2}$. For any $R>0$, define a cut-off function

$$
\eta_{R}: \mathbb{C} \rightarrow[0,1] \quad \eta_{R}(x):=\eta(x / R) .
$$

Let dvol $_{\text {Euc }}$ denote the Euclidean metric on $\mathbb{C} \subset \tilde{P}$ and dvol FS $_{\text {be }}$ be the Fubini-Study metric on $\tilde{\mathbb{P}}$. For any $R>2$, define a metric on $\operatorname{dvol}_{R}$ on $\tilde{\mathbb{P}}$ as

$$
\begin{aligned}
\mathrm{dvol}_{R} & :=\left(1-\eta_{R}\right) \mathrm{dvol}_{\mathrm{FS}}+\eta_{R}\left(1-\eta_{1 / 2 R}\right) \mathrm{dvol}_{\mathrm{orb}}+\eta_{1 / 2 R} n^{2} R^{-2 n+2} \mathrm{dvol}_{\mathrm{Euc}} \\
& =\left(\frac{1-\eta_{R}}{\left(1+x^{2}+y^{2}\right)^{2}}+\eta_{R}\left(1-\eta_{1 / 2 R}\right) n^{2}\left(x^{2}+y^{2}\right)^{n-1}+\eta_{1 / 2 R} n^{2} R^{-2 n+2}\right) d x \wedge d y,
\end{aligned}
$$

where $(x, y)$ are Euclidean coordinates on $\mathbb{C} \subset \tilde{\mathbb{P}}$. 
We remark that in case the git quotient is a manifold, $n=1$, the family of metrics simplifies to

$$
\mathrm{dvol}_{R}:=\left(1-\eta_{R}\right) \mathrm{dvol}_{\mathrm{FS}}+\eta_{R} \operatorname{dvol}_{\mathrm{Euc}}=\left(\frac{1-\eta_{R}}{\left(1+x^{2}+y^{2}\right)^{2}}+\eta_{R}\right) d x \wedge d y .
$$

The family of metrics described above satisfies the following uniform bound:

Lemma 3.13. Let $\Omega, B \subset \tilde{\mathbb{P}}$ be small open neighborhoods of 0 and $\infty$ respectively. Suppose $f_{R}: \tilde{\mathbb{P}} \rightarrow \mathbb{R}_{\geq 0}$ be given by the relation $\mathrm{dvol}_{R}=f_{R}$ dvol Fs $_{\text {. There exists a }}$ constant $c$ such that

$$
\left|f_{R}\right| \leq c \text { on } \Omega, \quad c^{-1} \leq\left|f_{R}\right| \text { on } B, \quad c^{-1} \leq\left|f_{R}\right| \leq c \text { on } \tilde{\mathbb{P}} \backslash(\Omega \cup B) .
$$

Proof. To prove the first relation, suppose $\Omega$ is contained in a ball of size $r$ about 0 . Consider values of $R$ for which $R>r>\frac{1}{R}$. On $\Omega, \eta_{R}=1$. So, we can write

$$
\begin{aligned}
\operatorname{dvol}_{R} & :=\left(1-\eta_{1 / 2 R}\right) \mathrm{dvol}_{\mathrm{orb}}+\eta_{1 / 2 R} n^{2} R^{-2 n+2} \operatorname{dvol}_{\text {Euc }} \\
& =\left(\left(1-\eta_{1 / 2 R}\right) n^{2}\left(x^{2}+y^{2}\right)^{n-1}+\eta_{1 / 2 R} n^{2} R^{-2 n+2}\right) d x \wedge d y .
\end{aligned}
$$

We introduce another function $g_{R}:=f_{R}\left(1+x^{2}+y^{2}\right)^{-2}$ on $\Omega$, so that $\operatorname{dvol}_{R}=g_{R} d x \wedge d y$. Then, $g_{R} \leq n^{2} r^{2 n-2}$ on $\Omega$, therefore there is an upper bound on $f_{R}$ also.

Next, consider the second relation. Suppose $r$ is such that $B$ is contained in $\tilde{\mathbb{P}} \backslash B_{r}$. Consider values of $R$ such that $\frac{1}{R}<r$. On $B$, the term $\eta_{1 / 2 R}$ is 0 , then,

$$
\begin{aligned}
\operatorname{dvol}_{R} & =\left(1-\eta_{R}\right) \mathrm{dvol}_{\mathrm{FS}}+\eta_{R} \mathrm{dvol}_{\mathrm{orb}} \\
& =\left(\frac{1-\eta_{R}}{\left(1+x^{2}+y^{2}\right)^{2}}+\eta_{R} n^{2}\left(x^{2}+y^{2}\right)^{n-1}\right) d x \wedge d y,
\end{aligned}
$$

Since dvol FS $_{\text {is }}$ smaller than dvol orb on $B$, we have $f_{R} \geq 1$.

The last relation follows from the fact that for large enough $R$, the metric $\mathrm{dvol}_{R}$ is equal to dvol orb on $\tilde{\mathbb{P}} \backslash(\Omega \cup B)$.

The following is a preparatory result required to prove the main result of this section (Proposition 3.10). It says that given a stable gauged holomorphic map on a weighted projective line, it can be complex gauge transformed in a way that its energy is uniformly bounded with respect to the family of metrics described above.

Proposition 3.14. Let $(A, u)$ be a gauged holomorphic map defined on a principal bundle $P \rightarrow \mathbb{P}(1, n)$, and suppose $u(\infty) \in X^{\mathrm{ss}}$. Then there exists a smooth complex gauge transformation $g \in \mathcal{G}(P)$ such that

$$
\sup _{R} E_{R}\left(\pi^{*}(g \cdot(A, u))\right)<\infty .
$$

Here $\pi: \tilde{\mathbb{P}} \rightarrow \mathbb{P}(1, n)$ is the projection and $E_{R}$ is the energy of the gauged holomorphic map with respect to the metric $\operatorname{dvol}_{R}$ on $\tilde{\mathbb{P}}$.

Proof. We first find a smooth $g \in \mathcal{G}(P)$ such that

$$
F_{g A} \equiv 0 \text { in a neighborhood of } 0, \quad \Phi(g u) \equiv 0 \text { in a neighborhood of } \infty .
$$


For the first condition, we use Theorem 6.3 on a neighborhood $\Omega$ of 0 in $\mathbb{P}(1, n)$. This produces a unique element $s: \Omega \rightarrow P(\mathfrak{k})$ satisfying $\left.s\right|_{\partial \Omega} \equiv 0$ such that $e^{i s} A$ is a flat connection on $\Omega$. The element $s$ is smooth by Lemma 3.18. By taking $\Omega$ to be a neighborhood of 0 , and using a cut-off function, we can produce $g_{1} \in \mathcal{G}(P)$ that makes the connection flat in a neighborhood of 0 and $g_{1}$ is identity away from this neighborhood. For the second condition $\Phi(g u)=0$, observe that there is a neighborhood $B$ of $\infty$ in $\mathbb{P}(1, n)$ that is mapped by $u$ to $X^{\mathrm{ss}}$. For any $x \in X^{\mathrm{ss}}$, there is a unique $s \in \mathfrak{k}$ such that $\Phi\left(e^{i s} x\right)=0$, and $s$ varies smoothly with $x$. Let $\tilde{B}:=\pi^{-1}(B) \subset \tilde{U}_{1}$ be the lift of $B$. We work with the trivialization of $P$ over $\tilde{U}_{1}$ as in (11). There exists $\xi: \tilde{B} \rightarrow \mathfrak{k}$ such that $\Phi\left(e^{i \xi} u\right) \equiv 0$ on $\tilde{B}$. Recall $u$ satisfies $\mathbb{Z}_{n}$-equivariance $u \circ \sigma_{n}=\mu u$. The uniqueness of $\xi$ implies

$$
\xi \circ \sigma_{n}=\operatorname{Ad}_{\mu} \xi \text {. }
$$

By using a cut-off function, $\xi$ can be extended to all of $\tilde{U}_{1}$ so that it vanishes away from $\tilde{B}$ and still satisfies the symmetry relation (24). The element $g:=g_{1} e^{i \xi}$ satisfies the conditions in (23), by construction.

Using Lemma 3.13, we compare the $R$-dependent metric with the Fubini-Study metric. Conformally rescaling the metric by a scalar-valued function greater than one has the effect of decreasing the $L^{2}$ norm of the two-form $F_{A}$ and increasing the $L^{2}$ norm of the zero-form $\Phi(u)$. The $L^{2}$ norm of the one-form $\mathrm{d}_{A} u$ is independent of the metric. By (23) we have that for any $R$,

$$
E_{R}\left(\pi^{*}(g \cdot(A, u))\right)<c E_{\mathrm{FS}}\left(\pi^{*}(g \cdot(A, u))\right.
$$

where $E_{\mathrm{FS}}$ denotes the energy defined using the Fubini-Study metric. Hence the complex gauge transformation $g$ satisfies the bound (22).

Proof. (Proof of Proposition 3.10 when the git quotient is a manifold) The proof of the first part of the proposition is by applying the heat flow Theorem 3.3 on the given stable gauged holomorphic map with respect to a sequence of metrics described in (21). Given a gauged holomorphic map $(A, u)$ on a principal bundle $P \rightarrow \mathbb{P}^{1}$ that satisfies $u(\infty) \in X^{\mathrm{ss}}$, by Proposition 3.14 we can assume

$$
E_{R}(A, u)<e_{0} \quad \forall R>0,
$$

where $E_{R}$ is the energy with respect to the metric $\operatorname{dvol}_{R}$. As $R$ increases, the volume $\operatorname{vol}_{R}\left(\mathbb{P}^{1}\right)$ increases to infinity. So, there exists $r_{0}$ such that $E_{R}(A, u) \leq e_{0} \leq$ $c_{0}^{2} \operatorname{vol}_{R}\left(\mathbb{P}^{1}\right)$ for all $R \geq r_{0}$, where $c_{0}$ is as defined in (13). By dropping some initial terms of the given sequence $\left\{R_{i}\right\}$, we have $R_{i} \geq r_{0}$ for all $i$. Theorem 3.3 is applicable for the gauged holomorphic map $(A, u)$ on $\mathbb{P}^{1}$ with respect to the metric $\mathrm{dvol}_{R_{i}}$. By Theorem 3.3, $(A, u)$ is complex gauge equivalent to a vortex $\left(A_{i}, u_{i}\right)$ with respect to the metric dvol $R_{i}$ via a complex gauge transformation $g_{i} \in \mathcal{G}(P)_{W^{2, p}}$. On the ball $B_{R_{i}},\left(A_{i}, u_{i}\right)$ is a vortex with respect to the Euclidean metric. By modifying each $g_{i}$ by a gauge transformation on $B_{R_{i}}$, we may assume that $\left(A_{i}, u_{i}\right)$ is smooth on $B_{R_{i}}$ (by [10, Theorem 3.1]). This finishes the proof of part (a) of the Proposition 3.10.

We now have a sequence of gauged holomorphic maps on the projective line whose restriction to a sequence of exhausting subsets of the complex line is a vortex with 
respect to the Euclidean metric. We show that the restrictions of the sequence of maps on these subsets satisfy the hypothesis for the Gromov convergence result (Lemma 3.7). The gauged maps $\left(A_{i}, u_{i}\right)$ are all homotopic to each other, so they have the same equivariant homology class $[u] \in H_{2}^{K}(X)$. Then, by [10, Lemma 2.7], there is a compact set $S \subset X$ that contains the images of all the $u_{i}$. The pair $\left(A_{i}, u_{i}\right)$, when restricted to $B_{R_{i}}$, is a vortex with respect to the Euclidean metric. It satisfies $E\left(A_{i}, u_{i}, B_{R_{i}}\right) \leq E_{R_{i}}\left(A_{i}, u_{i}, \mathbb{P}^{1}\right) \leq E_{R_{i}}\left(A, u, \mathbb{P}^{1}\right) \leq e_{0}$. The first inequality follows from the definition of the metric $\mathrm{dvol}_{R_{i}}$, the second one from the fact that heat flow decreases energy (see Lemma 3.4) and the last one follows from (25).

The proof of the Proposition can be completed by applying Gromov convergence for vortices (Lemma 3.7). The sequence $\left\{\left(A_{i}, u_{i}\right)\right\}$ is a sequence of vortices on $B_{R_{i}}$, which exhaust $\mathbb{C}$. The sequence satisfies an energy bound, so Lemma 3.7 is applicable. So, there is a subsequence of $\left(A_{i}, u_{i}\right)$ (still denoted by the same subscripts), a sequence of gauge transformations $k_{i} \in H^{2}\left(B_{R_{i}}\right)$, a finite set $Z \subseteq \mathbb{C}$ and a finite energy vortex $\left(A_{\infty}, u_{\infty}\right)$ on $\mathbb{C}$ so that $k_{i} A_{i} \rightarrow A_{\infty}$ in $H^{1}$ on compact subsets of $\mathbb{C}$ and $k_{i} u_{i} \rightarrow u_{\infty}$ in $H^{2}$ on compact subsets of $\mathbb{C} \backslash Z$.

Proof. (Proof of Proposition 3.10 when the git quotient is an orbifold.) We work with a lift of the given stable gauged holomorphic map to the ramified cover of the weighted projective line. We first state some symmetry properties of this lift. Suppose $(A, u)$ is the given gauged holomorphic map $(A, u)$ on a principal bundle $P \rightarrow \mathbb{P}(1, n)$. The lift of this map to the bundle $\pi^{*} P \rightarrow \tilde{\mathbb{P}}$ is denoted $(\tilde{A}, \tilde{u})$. A trivialization of the bundle $P \rightarrow \mathbb{P}(1, n)$ on the charts $\tilde{U}_{1}, U_{2}$ (see $(9)$ ) lifts to a trivialization of $\pi^{*} P \rightarrow \tilde{\mathbb{P}}$ on $\tilde{U}_{1}$ and $\tilde{U}_{2}$. We fix such a trivialization. On the chart $\tilde{U}_{2} \simeq \mathbb{C} \subset \tilde{\mathbb{P}}$, the lift $(\tilde{A}, \tilde{u})$ is symmetric under the $\mathbb{Z}_{n}$-action, or in other words $(\tilde{A}, \tilde{u})$ is preserved by $\frac{2 \pi}{n}$-rotations of the domain. On the trivialization over $\tilde{U}_{1} \simeq \tilde{\mathbb{P}} \backslash\{0\}$, we have $\sigma_{n}^{*}(\tilde{A}, \tilde{u})=\mu(\tilde{A}, \tilde{u})$.

We now produce a sequence of vortices on a sequence of exhausting annuli. We also prove energy bounds in this sequence so that Gromov convergence is applicable. We define another increasing sequence $\tilde{R}_{i}:=R_{i}^{1 / n}$, and remark that the annulus $A\left(1 / R_{i}, R_{i}\right)$ in $\mathbb{P}(1, n)$ lifts to the annulus $A\left(1 / \tilde{R}_{i}, \tilde{R}_{i}\right)$ in $\tilde{\mathbb{P}}$. By Proposition 3.14 , we can assume

$$
E_{\tilde{R}}(\tilde{A}, \tilde{u}, \tilde{\mathbb{P}})<e_{0} \quad \forall \tilde{R}>0,
$$

where $E_{\tilde{R}}$ denotes the energy with respect to the dvol $\tilde{R}_{\tilde{R}}$ metric. As in the case when the git quotient is a manifold, after dropping some terms in the sequence $\left\{\tilde{R}_{i}\right\}$, we may assume that $E_{\tilde{R}_{i}}(\tilde{A}, \tilde{u}) \leq e_{0} \leq c_{0}^{2} \operatorname{vol}_{\tilde{R}_{i}}(\tilde{\mathbb{P}})$, which ensures that Theorem 3.3 is applicable. Using Theorem 3.3, we get a sequence of complex gauge transformations $e^{i \tilde{\xi}_{i}} \in W^{2, p}\left(\mathcal{G}\left(\pi^{*} P\right)\right)$ such that $e^{i \tilde{\xi}_{i}}(\tilde{A}, \tilde{u})$ are $\mathrm{dvol}_{\tilde{R}_{i}}$-vortices on $\tilde{\mathbb{P}}$. The starting energy $E_{\tilde{R}_{i}}(A, u)$ is bounded and heat flow decreases energy (see Lemma 3.4). Therefore, the elements in the sequence $e^{i \tilde{\xi}_{i}}(\tilde{A}, \tilde{u})$ have bounded energy with respect to the metric $\operatorname{dvol}_{\tilde{R}_{i}}$. Further, the gauged maps $e^{i \tilde{\xi}_{i}}(\tilde{A}, \tilde{u})$ are homotopic to each other, so by [10, Lemma 2.7], there is a compact set $S \subset X$ that contains the images of the maps $e^{i \tilde{\xi}_{i}} \tilde{u}$. By Theorem 3.3, the element $\tilde{\xi}_{i}$ is unique, so the complex 
gauge transformation $e^{i \tilde{\xi}_{i}}$ is symmetric under the $\mathbb{Z}_{n}$ action, and descends to the weighted projective line, we denote $\xi_{i}:=\pi_{*} \tilde{\xi}_{i}$. The same is therefore true of the pair $e^{i \tilde{\xi}_{i}}(\tilde{A}, \tilde{u})$, and it descends to the pair $e^{i \xi_{i}}(A, u)$ defined on $\mathbb{P}(1, n)$. The metric $\operatorname{dvol}_{\tilde{R}_{i}}$ descends to the Euclidean metric on the annulus $A\left(1 / R_{i}, R_{i}\right) \subset \mathbb{P}(1, n)$, so $e^{i \xi_{i}}(A, u)$ is a vortex with respect to the Euclidean metric on this annulus with an energy bound:

$$
E\left(e^{i \xi_{i}}(A, u) ; A\left(1 / R_{i}, R_{i}\right)\right)=\frac{1}{n} E_{\operatorname{dvol}_{\tilde{R}_{i}}}\left(e^{i \tilde{\xi}_{i}}(\tilde{A}, \tilde{u}) ; A\left(1 / \tilde{R}_{i}, \tilde{R}_{i}\right)\right) \leq e_{0} / n .
$$

However, in the neighborhood of the origin, we do not have control over the $L^{2}$ norm of the curvature with respect to the Euclidean metric. We apply Proposition 3.19 to the sequence $e^{i \tilde{\xi}_{i}}(\tilde{A}, \tilde{u})$ on $\tilde{\mathbb{P}}$. This gives a sequence of $H^{2}$ complex gauge transformations $e^{i \tilde{s}_{i}}$ on $\tilde{\mathbb{P}}$ supported in the balls $B_{1 / \tilde{R}_{i}}$ that are $\mathbb{Z}_{n}$-symmetric and satisfy the following: if $s_{i}:=\pi_{*} \tilde{s}_{i}: \mathbb{C} \rightarrow \mathfrak{k}$, then the curvature norms $\left\|F_{e^{i s_{i}}} e^{i \xi_{i} A}\right\|_{L^{2}}$ are uniformly bounded. Part (a) of Proposition 3.10 is proved by the complex gauge transformations $g_{i}:=e^{i s_{i}} e^{i \xi_{i}}: B_{R_{i}} \rightarrow G$. Set $\left(A_{i}, u_{i}\right):=g_{i}(A, u)$ on $B_{R_{i}}$. Since $s_{i}$ is supported in $B_{1 / R_{i}}$, on the annulus $A\left(1 / R_{i}, R_{i}\right),\left(A_{i}, u_{i}\right)=g_{i}(A, u)$ is still a vortex that satisfies

$$
E\left(A_{i}, u_{i} ; A\left(1 / R_{i}, R_{i}\right)\right) \leq c, \quad\left\|F_{A_{i}}\right\|_{L^{2}\left(B_{1 / R_{i}}\right)} \leq c .
$$

We apply Gromov convergence on the sequence of vortices constructed above that are defined on a sequence of exhausting annuli. Combining the equations in (27), we get a uniform bound on the energy of the connections $\left\|F\left(A_{i}\right)\right\|_{L^{2}\left(B_{R_{i}}\right)}^{2}$. By Uhlenbeck compactness for non-compact domains ( $[45$, Theorem A']), after passing to a subsequence, there is a sequence of gauge transformations $k_{i} \in H^{2}\left(B_{R_{i}}\right)$ and a connection $A_{\infty}$ on the trivial bundle $\mathbb{C} \times K$ such that $k_{i} A_{i} \rightarrow A_{\infty}$ in $H^{1}$ in compact subsets of $\mathbb{C}$. We apply Lemma 3.7 to the sequence of vortices on domains $B_{R_{i}} \backslash B_{1 / R_{i}}$. The Lemma implies that there is a finite set $Z \subset \mathbb{C} \backslash\{0\}$ and a finite energy limit vortex $\left(A_{\infty}, u_{\infty}\right)$, where $A_{\infty}$ is the limit connection found earlier and $u_{\infty}$ is defined on $\mathbb{C} \backslash\{0\}$ such that $k_{i} u_{i} \rightarrow u_{\infty}$ in $H^{2}$ on compact subsets of $\mathbb{C} \backslash(Z \cup\{0\})$.

To finish the proof of the Proposition, it remains to remove the singularity of the limit map at the origin. Choose a small positive number $r>0$. The pair $\left(A_{\infty}, u_{\infty}\right)$ is a vortex on $B_{r} \backslash\{0\}$. Since the image $k_{i} u_{i}\left(B_{r} \backslash B_{R_{i}}\right)$ is contained in a compact set $S \subset X$ for all $i$, the image of $u_{\infty}$ is also contained in $S$. By Lemma 4.3, there is a complex gauge transformation $g \in H^{2}\left(B_{r}\right)$ such that $g A_{\infty}$ is the trivial connection. Complex gauge transformations preserve holomorphicity, so $\bar{\partial}\left(g u_{\infty}\right)=0$ on $B_{r} \backslash\{0\}$ and the image $g u_{\infty}\left(B_{R} \backslash\{0\}\right)$ is contained in a compact set. Further $g u_{\infty}$ is smooth. By the removable singularity theorem of complex analysis, the map $g u_{\infty}$ extends smoothly over 0 , and hence $u_{\infty}$ extends over 0 as a $H^{2}$ map.

3.6. Finding complex gauge transformations to relate nearby vortices. Given a sequence of converging vortices on a ball in the complex plane that are in the same complex gauge equivalence class, we address the question of whether 
the limit modulo bubbling of the sequence is also in the same complex gauge equivalence class. As part of the hypothesis, we are given that the limit is in the same complex gauge equivalence class in an annulus. In particular the limit is determined by a limit complex gauge transformation. In the following proposition we extend the limit complex gauge transformation to the entire ball. If one naively tries to take the limit of complex gauge transformations generating the sequence of vortices, one is obstructed by (a) the bubbling points (represented by $Z$ below) and (b) points in a semistable orbit coverging to an unstable point in the closure of the orbit.

Proposition 3.15. Let $0<r<R$ and $B_{r} \subset B_{R} \subset \mathbb{C}$ be open balls of radii $r$ and $R$. Let $Z \subset B_{r}$ be a finite set and $\left(A_{i}, u_{i}\right)$ be a sequence of vortices in class $\left(L^{p} \times W^{1, p}\right)\left(B_{R}\right)$ that satisfy the following.

(a) The sequence $\left(A_{i}, u_{i}\right)$ converges to a vortex $\left(A_{\infty}, u_{\infty}\right) \in\left(L^{p} \times W^{1, p}\right)\left(B_{R}\right)$ in the following sense:

$$
A_{i} \stackrel{L^{p}\left(B_{R}\right)}{\longrightarrow} A_{\infty}, \quad u_{i} \stackrel{W^{1, p}(Q)}{\longrightarrow} u_{\infty} \text { for all compact subsets } Q \subset B_{R} \backslash Z .
$$

(b) There are complex gauge transformations $g_{i} \in W^{1, p}\left(B_{R}, G\right)$ such that $g_{i}\left(A_{0}, u_{0}\right)=$ $\left(A_{i}, u_{i}\right)$.

(c) There is also a complex gauge transformation $g_{\infty}: \bar{B}_{R} \backslash B_{r} \rightarrow G$ such that $g_{i}$ converges to $g_{\infty}$ in $W^{1, p}\left(B_{R} \backslash B_{r}\right)$ and $\left(A_{\infty}, u_{\infty}\right)=g_{\infty}\left(A_{0}, u_{0}\right)$ on $\bar{B}_{R} \backslash B_{r}$.

(d) For all $i$, including $i=\infty, u_{i}\left(\bar{B}_{R} \backslash B_{r}\right) \subset X^{\mathrm{ss}}$.

Then, the complex gauge transformation $g_{\infty}$ can be defined on all of $\bar{B}_{R}$ in a way that $\left(A_{\infty}, u_{\infty}\right)=g_{\infty}\left(A_{0}, u_{0}\right)$ and the sequence $g_{i}$ converges to $g_{\infty}$ weakly in $W^{1, p}\left(B_{R}\right)$.

Proof. In Step 1 and 2 of the proof, we adjust the sequence of vortices, via a sequence of small complex gauge transformations, to produce a new sequence of vortices that are equal to each other on the boundary upto unitary gauge equivalence. Then, in Step 3, we show that the vortices in the new sequence, which still has the same limit, are related to each other by unitary gauge transformations. Finally, in Step 4 , by a gauge theoretical argument, we find a limit of the unitary gauge transformations, that indeed corresponds to the limit vortex. Let $N\left(\partial B_{R}\right)$ denote a closed neighborhood of $\partial B_{R}$ disjoint from $\bar{B}_{r}$.

STEP 1: There exists a sequence of complex gauge transformations $g_{i}^{\prime} \in W^{1, p}\left(B_{R}, G\right)$, each of whose terms is respectively equal to $g_{\infty} g_{i}^{-1}$ on $N\left(\partial B_{R}\right)$ and is equal to $\mathrm{Id}$ on $B_{r}$. Further $g_{i}^{\prime} \rightarrow$ Id in $W^{1, p}\left(B_{R}, G\right)$. Consequently, the gauged holomorphic maps $\left(A_{i}^{\prime}, u_{i}^{\prime}\right):=g_{i}\left(A_{i}, u_{i}\right)$ are equal to $\left(A_{\infty}, u_{\infty}\right)$ on $N\left(\partial B_{R}\right)$ and $\left(A_{i}^{\prime}, u_{i}^{\prime}\right)$ is equal to $\left(A_{i}, u_{i}\right)$ on $B_{r}$.

By the hypothesis of the proposition, $g_{\infty} g_{i}^{-1}$ converges to Id in $W^{1, p}\left(B_{R} \backslash B_{r}\right)$. So, for large $i$, the image of $g_{\infty} g_{i}^{-1}$ is contained in a neighborhood of Id where the exponential map $e: \mathfrak{g} \rightarrow G$ is injective. This means we can write $e^{\zeta_{i}}=g_{\infty} g_{i}^{-1}$, where $\zeta_{i}: \bar{B}_{R} \backslash B_{r} \rightarrow \mathfrak{g}$. Let $\eta: \bar{B}_{R} \rightarrow[0,1]$ be a cut-off function that is equal to 1 in a neighborhood of $\partial B_{R}$ and is equal to 0 on $B_{r}$. The required complex gauge transformations are $g_{i}^{\prime}:=e^{i \eta \zeta_{i}}$. 
STEP 2: There exists a sequence $\xi_{i} \in W^{1, p}\left(B_{R}, \mathfrak{k}\right)$ so that $\left.\xi_{i}\right|_{\partial B_{R}}=0$ and the gauged holomorphic maps $\left(A_{i}^{\prime \prime}, u_{i}^{\prime \prime}\right):=e^{i \xi_{i}}\left(A_{i}^{\prime}, u_{i}^{\prime}\right)$ are vortices on $\bar{B}_{R}$. Further $\xi_{i} \rightarrow 0$ in $W^{1, p}\left(B_{R}, \mathfrak{k}\right)$.

This result is obtained by applying Proposition 3.5 on the sequence $\left(A_{i}^{\prime}, u_{i}^{\prime}\right)$ defined on $\bar{B}_{R}$. First we check the hypothesis. The convergence of $\left(A_{i}, u_{i}\right)$ in $(28)$ together with the condition $g_{i}^{\prime} \rightarrow \operatorname{Id}$ in $W^{1, p}\left(B_{R}, G\right)$ guarantees that the following is true:

$$
A_{i}^{\prime} \stackrel{L^{p}\left(B_{R}\right)}{\longrightarrow} A_{\infty}, \quad u_{i}^{\prime} \stackrel{W^{1, p}(Q)}{\longrightarrow} u_{\infty} \text { for all compact subsets } Q \subset \bar{B}_{R} \backslash Z .
$$

We also have $F_{A_{i}^{\prime}, u_{i}^{\prime}}($ recall terminology from $(7))$ converges to 0 in $W^{-1, p}$. This is because $\left(A_{i}^{\prime}, u_{i}^{\prime}\right)$ is a vortex on $B_{r}$ and on $\bar{B}_{R} \backslash B_{r}$, the convergence follows using (29) and the fact that $Z \subset B_{r}$. Step 2 is proved by the conclusions of Proposition 3.5.

STEP 3: The vortices in the sequence $\left(A_{i}^{\prime \prime}, u_{i}^{\prime \prime}\right)$ are unitary gauge equivalent to each other.

The vortices $\left(A_{i}^{\prime \prime}, u_{i}^{\prime \prime}\right)$ are complex gauge equivalent to each other, in particular, on $\bar{B}_{R}$

$$
\left(A_{i}^{\prime \prime}, u_{i}^{\prime \prime}\right)=h_{i}\left(A_{0}, u_{0}\right), \quad \text { where } h_{i}:=e^{i \xi_{i}} g_{i}^{\prime} g_{i} \in W^{1, p}\left(B_{R}, G\right) .
$$

By the construction of $g_{i}^{\prime}$ in Step 1 , we know that $g_{i}^{\prime} g_{i}$ is equal to $g_{\infty}$ in $N\left(\partial B_{R}\right)$. Together with the fact that $\left.\xi_{i}\right|_{\partial B_{R}}=0$, we can say that $h_{i} h_{j}^{-1}\left(\partial B_{R}\right) \subset K$ for any $i, j$. But then, by Proposition 3.6, the vortices are gauge equivalent, in particular $h_{i} h_{j}^{-1}\left(\bar{B}_{R}\right) \subset K$ for all $i, j$.

STEP 4: Finishing the proof.

First, we claim that $A_{i}^{\prime \prime} \rightarrow A_{\infty}$ in $L^{p}\left(B_{R}\right)$. This follows from the convergence of $A_{i}$, $g_{i}^{\prime}$ and $\xi_{i}$ in the spaces $L^{p}, W^{1, p}$ and $W^{1, p}$ respectively, and by using the continuous action of $\mathcal{G}(P)$ on $\mathcal{A}(P)$ (see Lemma 6.4). We can write $\left(A_{i}^{\prime \prime}, u_{i}^{\prime \prime}\right)=h_{i}^{\prime}\left(A_{0}^{\prime \prime}, u_{0}^{\prime \prime}\right)$ and we know from Step 3 that $h_{i}^{\prime}=h_{i} h_{0}^{-1}$ and that it is a unitary gauge transformation. By a standard gauge theoretic argument (Lemma 6.5), after passing to a subsequence $h_{i}^{\prime} \rightarrow h_{\infty}^{\prime}$ in $W^{1, p}\left(B_{R}\right)$ (and strongly in $\left.C^{0}\right), A_{\infty}=h_{\infty}^{\prime} A_{0}^{\prime \prime}$. This implies that the sequence $u_{i}^{\prime \prime}$, which is same as $h_{i}^{\prime} u_{0}^{\prime \prime}$, converges to $h_{\infty}^{\prime} u_{0}^{\prime \prime}$ weakly in $W^{1, p}\left(B_{R}\right)$. Also, $u_{i}^{\prime \prime}$ converges in $W^{1, p}$ to $u_{\infty}$ on compact subsets of $\bar{B}_{R} \backslash Z$, so $u_{\infty}=h_{\infty}^{\prime} u_{0}^{\prime \prime}$. Therefore, on $\bar{B}_{R}$, using Figure 2,

$$
\left(A_{\infty}, u_{\infty}\right)=h_{\infty}^{\prime}\left(A_{0}^{\prime \prime}, u_{0}^{\prime \prime}\right)=h_{\infty}^{\prime} e^{i \xi_{0}} g_{0}^{\prime} g_{0}\left(A_{0}, u_{0}\right)
$$

To finish the proof, we show that the complex gauge transformation $h_{\infty}^{\prime} e^{i \xi_{0}} g_{0}^{\prime} g_{0}$ is the weak $W^{1, p}\left(B_{R}\right)$ limit of the sequence $g_{i}$. Working in the weak $W^{1, p}\left(B_{R}\right)$-topology, we can write

$$
h_{\infty}^{\prime} e^{i \xi_{0}} g_{0}^{\prime} g_{0}=\lim _{i} h_{i}^{\prime} e^{i \xi_{0}} g_{0}^{\prime} g_{0}=\lim _{i}\left(e^{i \xi_{i}} g_{i}^{\prime} g_{i}\right) h_{0}^{-1} e^{i \xi_{0}} g_{0}^{\prime} g_{0}=\lim _{i}\left(e^{i \xi_{i}} g_{i}^{\prime} g_{i}\right) .
$$

Since the terms $g_{i}^{\prime}$ and $e^{i \xi_{i}}$ converge to Id in $W^{1, p}\left(B_{R}\right)$, we have proved that $h_{\infty}^{\prime} e^{i \xi_{0}} g_{0}^{\prime} g_{0}$ is the weak $W^{1, p}\left(B_{R}\right)$-limit of the sequence $g_{i}$. So, $g_{\infty}:=h_{\infty}^{\prime} e^{i \xi_{0}} g_{0}^{\prime} g_{0}$ is an extension of the limit complex gauge transformation to all of $B_{R}$ and $g_{\infty}\left(A_{0}, u_{0}\right)=$ $\left(A_{\infty}, u_{\infty}\right)$. 


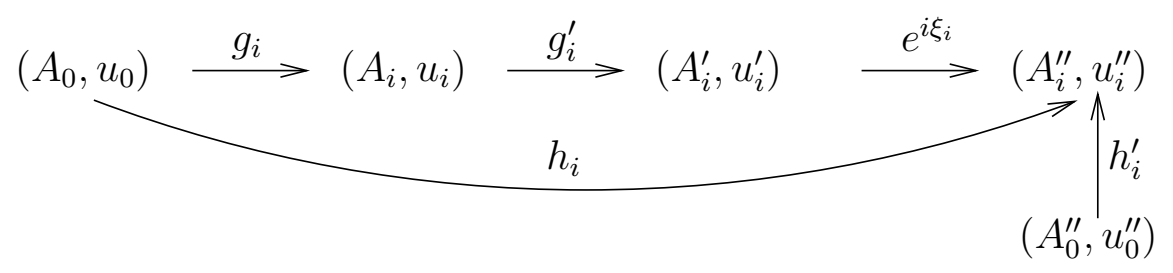

Figure 2. Complex gauge transformations used in proof of Proposition 3.15 .

3.7. Some results involving complex gauge transformations. The following sequence of Lemmas 3.16 - 3.18 were used in the proof of Theorem 3.1 (a). Lemma 3.16 says that a sequence of converging complex gauge equivalent maps to the semistable locus, after passing to a subsequence, is generated by a converging sequence of complex gauge transformations.

Lemma 3.16. (Converging maps are related by converging complex gauge transformations) Suppose $\Sigma \subseteq \mathbb{C}$ be the closure of a bounded open subset with smooth boundary and let maps $u_{i}: \Sigma \rightarrow X^{\mathrm{ss}}$ converge in $W^{m, p}$ to $u_{\infty}: \Sigma \rightarrow X^{\mathrm{ss}}$ for some $m, p$ satisfying $m p>2$. Assume further that the maps are related by complex gauge transformations i.e. there exist $g_{i} \in W^{m, p}(\Sigma, G)$ such that $u_{i}=g_{i} u_{0}$. Then, after passing to a subsequence $g_{i}$ converges to a limit $g_{\infty}$ in $W^{m, p}$ and $g_{\infty} u_{0}=u_{\infty}$.

Proof. Locally, the Lemma is proved by using a slice of the group action on the target space. Let $x \in X / / G$ and $y \in \pi_{G}^{-1}(x)$. We use a $G_{y}$-invariant slice $V \subset X^{\mathrm{ss}}$ containing $y$ such that there is a diffeomorphism $G \times \times_{G_{y}} V \rightarrow G V$. So, $G \times V \rightarrow G V$ is a $\left|G_{y}\right|$-cover. Suppose $U \subset \Sigma$ is such that $u_{\infty}(U) \subset V, u_{i}(U) \subset V$ for large $i$. Choose lifts $\tilde{u}_{i}=\left(\tilde{g}_{i}, v_{i}\right): U \rightarrow G \times V$ so that $\tilde{g}_{i}^{-1} \tilde{g}_{j}=\left.g_{i}^{-1} g_{j}\right|_{U}$. Since $u_{i} \rightarrow u_{\infty}$, a subsequence of $\tilde{u}_{i}$ converges in $W_{\text {loc }}^{m, p}$. (This is because: $G \times V \rightarrow G V$ is a local diffeomorphism and has smooth inverses locally.) So, $\tilde{g}_{i}$ converges and consequently $g_{i}$ converges to a limit $g_{\infty}: U \rightarrow G$. The set $\Sigma$ can be covered by a countable number of sets of the form of $U$. Working with the chosen cover of $\Sigma$, by successively passing to subsequences and using a diagonalization argument, we obtain a limit $g_{\infty}$ on all of $\Sigma$. By the smooth action of $G$ on $X^{\text {ss }}$, we get $u_{\infty}=g_{\infty} u_{0}$.

The next Lemma shows that for two gauged holomorphic pairs whose image lies in the semistable locus, if the maps are related by a complex gauge transformation, then the same is true of the connections also.

Lemma 3.17. Let $\Sigma \subset \mathbb{C}$ and $\left(A_{i}, u_{i}\right)$ be vortices on $\Sigma$ for $i=1,2$ such that $u_{i}(\Sigma) \subset X^{\mathrm{ss}}$. Suppose there is a complex gauge transformation $g: \Sigma \rightarrow G$ such that $g u_{1}=u_{2}$. Then, $g A_{1}=A_{2}$.

Proof. The proof relies on the free infinitesimal action of the group on the target space of the maps.

$$
\mathfrak{k}_{x} \cap J \mathfrak{k}_{x}=\{0\} .
$$


Let $a:=g A_{1}-A_{2}$. Since both $\left(g A_{1}, u_{2}\right)$ and $\left(A_{2}, u_{2}\right)$ are holomorphic, we have $\bar{\partial}_{g A_{1}} u_{2}-\bar{\partial}_{A_{2}} u_{2}=a_{u_{2}}^{0,1}=0$. Write $a=a_{x} d x+a_{y} d y, a_{x}, a_{y}: \Sigma \rightarrow \mathfrak{k}$. Then, $a_{x}\left(u_{2}\right)+J a_{y}\left(u_{2}\right)=0$ and by $(30), a=0$ on $\Sigma$.

Lemma 3.18. (Regularity of complex gauge transformations) Let $k \in \mathbb{Z}_{\geq 0}$ and $p>1$ be such that $k p>2$. Let $\Sigma$ be a compact Riemann surface and $P \rightarrow \Sigma$ be a principal $K$-bundle, and let $P_{\mathbb{C}}:=P \times_{K} G$ be the associated $G$-bundle. Suppose $A \in \mathcal{A}(P)$ is a smooth connection and $g \in \mathcal{G}(P)_{k, p}$ a complex transformation such that $g A$ is also smooth. Then $g$ is smooth.

Proof. We use the relation on $P_{\mathbb{C}}: \bar{\partial}_{g A}=g \circ \bar{\partial}_{A} \circ g^{-1}$. The difference $a:=g A-A$ is smooth and

$$
\bar{\partial}_{g A}-\bar{\partial}_{A}=a^{0,1}=g \bar{\partial}_{A}\left(g^{-1}\right)=-\left(\bar{\partial}_{A} g\right) g^{-1} .
$$

Therefore $a^{0,1} g=-\bar{\partial}_{A} g$. The smoothness of $g$ follows by elliptic bootstrapping.

3.8. A technical result. The result presented in this section is required only in the case of an orbifold git quotient and is used in the proof of Proposition 3.10 above. In transforming a stable gauged holomorphic map to an affine vortex, we recall that our first step was to produce a sequence of vortices on a sequence of exhausting annuli. This was done using heat flow on a ramified cover of the weighted projective line. The lift of the Euclidean metric on the affine line degenerates at the ramification point, which is chosen to be 0 . So the sequence of metrics used to run the heat flow is altered near the origin, the new sequence is called $\mathrm{dvol}_{R}$. The result of heat flow is a sequence of $\mathrm{dvol}_{R}$-vortices. Now, when the norm of the curvature is taken with respect to the lift of the Euclidean metric, it is no longer bounded. Therefore, the $\mathrm{dvol}_{R}$-vortices have to be altered by a sequence of complex gauge transformations supported near the origin. This alteration, carried out in the Proposition below, makes the $L^{2}$ norm of the curvature bounded with respect to the lift of the Euclidean metric. This is useful in removing the singularity of the limit vortex at the origin.

Proposition 3.19. Let $R_{i} \rightarrow \infty$ be an increasing sequence and $\tilde{R}_{i}:=R_{i}^{1 / n}$. Let $\left(\tilde{A}_{i}, \tilde{u}_{i}\right)$ be a sequence of $\operatorname{dvol}_{\tilde{R}_{i}}$-vortices on the $K$-bundle $\pi^{*} P \rightarrow \tilde{\mathbb{P}}$ that are symmetric under the $\mathbb{Z}_{n}$-action, whose energies are bounded and there is a compact set that contains the images of the maps $\tilde{u}_{i}$. There exist complex gauge transformations $e^{i \tilde{s}_{i}} \in W_{\text {loc }}^{2, p}\left(\mathcal{G}\left(\pi^{*} P\right)\right)$ that are symmetric under the $\mathbb{Z}_{n}$-action, are equal to identity on $\tilde{\mathbb{P}} \backslash B_{1 / \tilde{R}_{i}}$ and for which

$$
\sup _{i}\left\|F_{\pi_{*}\left(e^{i \tilde{s}_{i}} \tilde{A}_{i}\right)}\right\|_{L^{2}\left(B_{R_{i}}\right)}<\infty .
$$

The complex gauge transformations satisfy $\left\|\tilde{s}_{i}\right\|_{C^{0}\left(B_{1 / R_{i}}, \mathfrak{k}\right)}<c$.

Proof. We explain the strategy of the proof. The Euclidean metric on the affine line in $\mathbb{P}(1, n)$ corresponds to the orbifold metric dvol orb on the cover $\tilde{\mathbb{P}}$. For the given sequence of vortices, the curvature norm is bounded with respect to the dvol $\tilde{R}_{i}$ metric, which near the origin is bigger than the orbifold norm. Rescaling the metric by a factor smaller than one has the effect of increasing the $L^{2}$ norm of the curvature (a 
two-form). The Proposition is proved by a sequence of complex gauge transformations that make the connection flat in a small neighborhood (with radius of $1 / 2 \tilde{R}_{i}$ ) of zero.

We first produce the sequence of complex gauge transformations. We are working on balls in $\tilde{\mathbb{P}}$ about the origin with radius $1 / \tilde{R}_{i}$. But, since we need uniform bounds on the sequence of complex gauge transformations, it is necessary to use the same domain to construct each element of the sequence. This is done by dilating the domains to a ball of constant radius. For any $R>0$, let $\tilde{B}_{R} \subset \tilde{U}_{2}$ be the ball of radius $R$ centered at 0 in $\tilde{\mathbb{P}}$. Let

$$
\sigma_{R}: \tilde{B}_{1} \rightarrow \tilde{B}_{1 / R} \quad x \mapsto x / R
$$

be the dilation map. In the proof of this Proposition, $c$ will denote a constant that is independent of $i$, whose value may vary across appearances. In this proof, we use the symbol " $\approx$ " in the following sense: for two real non-negative $i$-dependent quantities $A, B$, we say $A \approx B$ if there exist constants $c_{1}$ and $c_{2}$ independent of $i$ such that $c_{1} A \leq B \leq c_{2} A$. We have

$$
\begin{aligned}
& \left\|\sigma_{\tilde{R}_{i}}^{*} F\left(\tilde{A}_{i}\right)\right\|_{L^{2}\left(\tilde{B}_{1}\right)}=\frac{1}{\tilde{R}_{i}}\left\|F\left(\tilde{A}_{i}\right)\right\|_{L^{2}\left(\tilde{B}_{1 / \tilde{R}_{i}}\right)}^{E u c} \approx \tilde{R}_{i}^{-2 n+1}\left\|F\left(\tilde{A}_{i}\right)\right\|_{L^{2}\left(\tilde{B}_{1 / \tilde{R}_{i}}\right)}^{\mathrm{dvol}_{\tilde{R}_{i}}} \\
& =\tilde{R}_{i}^{-2 n+1}\left\|\Phi\left(u_{i}\right)\right\|_{L^{2}\left(\tilde{B}_{1 / \tilde{R}_{i}}\right)}^{\mathrm{dvol}_{\tilde{R}_{i}}} \leq c \tilde{R}_{i}^{-3 n+1} .
\end{aligned}
$$

For the second equality, we use the fact that in this region, dvol $_{R_{i}}$ is the Euclidean metric scaled by a factor of $R_{i}^{-2 n+2}$ times a positive function with values between 1 and $2^{2 n-2}$. The third equality follows from the fact that $\left(\tilde{A}_{i}, \tilde{u}_{i}\right)$ is a $\operatorname{dvol}_{\tilde{R}_{i}}$-vortex. For the last inequality, we observe that the images of $u_{i}$ are contained in a compact subset of $X$ and hence there is a $C^{0}$ bound on $\Phi\left(u_{i}\right)$, so the bound on the $L^{2}$ norm has a factor of the square-root of the volume of the domain. By Uhlenbeck's local theorem ([42, Theorem 2.1]), for large $i$, we can now choose a trivialization corresponding to each connection $\sigma_{\tilde{R}_{i}}^{*} \tilde{A}_{i}$, under which the connection matrices are uniformly bounded and are invariant under the $\mathbb{Z}_{n}$-action, i.e.

$$
\left\|\sigma_{\tilde{R}_{i}}^{*} \tilde{A}_{i}\right\|_{H^{1}\left(\tilde{B}_{1}\right)} \leq c \tilde{R}_{i}^{-3 n+1} .
$$

We remark that this requires a symmetric version of the local theorem that given a symmetric connection, there exists a symmetric gauge transformation that puts the connection in Coulomb gauge. The symmetric version can be proved in a straightforward way by going through the steps of the proof of the local theorem. By Lemma 4.3 and Remark 4.4, there is a constant $c$ such that for large enough $i$, there exists $\tilde{\xi}_{i} \in H^{2}\left(\tilde{B}_{1 / \tilde{R}_{i}}, \mathfrak{k}\right)$ such that $\left.\tilde{\xi}_{i}\right|_{\partial B_{1 / \tilde{R}_{i}}}=0$, the connection $e^{i \tilde{\xi}_{i}}\left(\tilde{A}_{i}\right)$ is flat and

$$
\left\|\sigma_{\tilde{R}_{i}}^{*} \tilde{\xi}_{i}\right\|_{H^{2}\left(\tilde{B}_{1}\right)} \leq c\left\|\sigma_{\tilde{R}_{i}}^{*} F\left(\tilde{A}_{i}\right)\right\|_{L^{2}\left(\tilde{B}_{1}\right)} \leq c \tilde{R}_{i}^{-3 n+1} .
$$

By the uniqueness of $\tilde{\xi}_{i}$, it is symmetric under the $\mathbb{Z}_{n}$-action. The bound (33) implies a $C^{0}$ bound

$$
\left\|\tilde{\xi}_{i}\right\|_{C^{0}} \leq c \tilde{R}_{i}^{-3 n+1}
$$


and this bound is independent of metric. We cut-off $e^{i \tilde{\xi}_{i}}$ outside a ball of radius $1 / \tilde{R}_{i}$ and define $\tilde{s}_{i}:=\eta_{1 / 2} \tilde{R}_{i} \tilde{\xi}_{i}$, where the cut-off function $\eta_{1 / 2} \tilde{R}_{i}$ is as defined in $(20)$. The required sequence of complex gauge transformations is $e^{i \tilde{s}_{i}}$. The elements of the sequence inherit the $C^{0}$ bound on $\tilde{\xi}_{i}$ from (34).

We show that the curvature bound in the Proposition is satisfied with the complex gauge transformations $e^{i \tilde{s}_{i}}$ chosen above. We continue to work with the dilated quantities $\sigma_{R}^{*} \tilde{A}_{i}, \sigma_{R}^{*} \tilde{s}_{i}$ defined on the unit ball $\tilde{B}_{1} \subset \tilde{\mathbb{P}}$. The $H^{2}$ bound on $\sigma_{R}^{*} \tilde{\xi}_{i}$ implies

$$
\left\|\sigma_{\tilde{R}_{i}}^{*} \tilde{s}_{i}\right\|_{H^{2}\left(\tilde{B}_{1}\right)} \leq c \tilde{R}_{i}^{-3 n+1} .
$$

For large $i$, the estimate in Lemma 6.4 is applicable for the action of the complex gauge transformations $\sigma_{\tilde{R}_{i}}^{*} \tilde{s}_{i}$ on the connections $\sigma_{\tilde{R}_{i}}^{*} \tilde{A}_{i}$. Using (35) and (32) alongwith Lemma 6.4 , we can conclude

$$
\left\|\sigma_{\tilde{R}_{i}}^{*}\left(e^{i \tilde{s}_{i}} \tilde{A}_{i}-\tilde{A}_{i}\right)\right\|_{H^{1}\left(\tilde{B}_{1}\right)}<c \tilde{R}_{i}^{-3 n+1} .
$$

The curvature map $H^{1} \ni A \mapsto F_{A} \in L^{2}$ is continuous. So,

$$
\left\|\sigma_{R}^{*}\left(F_{e^{i \tilde{s}_{i}} \tilde{A}_{i}}-F_{\tilde{A}_{i}}\right)\right\|_{L^{2}\left(\tilde{B}_{1}\right)} \leq c\left\|\sigma_{\tilde{R}_{i}}^{*}\left(e^{\tilde{s}_{i}} \tilde{A}_{i}-\tilde{A}_{i}\right)\right\|_{H^{1}\left(\tilde{B}_{1}\right)}<c \tilde{R}_{i}^{-3 n+1} .
$$

In the above equation, the contribution of quadratic terms involving the connection can be ignored by adjusting the constant $c$, because the term $e^{\tilde{s}_{i}} \tilde{A}_{i}-\tilde{A}_{i}$ has small norms for large $i$ and so, the quadratic term is domainated by the linear term. Combining with (31), we get

$$
\left\|\sigma_{R}^{*} F_{e^{i \tilde{s}_{i}} \tilde{A}_{i}}\right\|_{L^{2}\left(\tilde{B}_{1}\right)}<c \tilde{R}_{i}^{-3 n+1} .
$$

Finally we transport the bound on the curvature on $\tilde{B}_{1}$ to the ball $B_{R_{i}} \subset \mathbb{P}(1, n)$. The quantities $\tilde{s}_{i}$ and $\tilde{A}_{i}$ are symmetic under the $\mathbb{Z}_{n}$-action, so they descend via $\pi_{*}$ to $\mathbb{P}(1, n)$. Define

$$
s_{i}:=\pi_{*} \tilde{s}_{i}, A_{i}:=\pi_{*} \tilde{A}_{i} .
$$

We recall that $\pi$ maps the ball $\tilde{B}_{\tilde{R}_{i}}$ to $B_{R_{i}}$ in $\mathbb{P}(1, n)$. By construction of $s_{i}$, the connection $e^{i s_{i}} A_{i}$ is flat in $B_{2^{-n} / R_{i}}$. So,

$$
\begin{aligned}
\left\|F_{e^{i s_{i}} A_{i}}\right\|_{L^{2}\left(B_{1 / R_{i}}\right)}^{\mathrm{Euc}} & =\left\|F_{e^{i s_{i}} A_{i}}\right\|_{L^{2}\left(A\left(2^{-n} / R_{i}, 1 / R_{i}\right)\right)}^{\mathrm{Euc}}=\left\|F_{e^{i \tilde{s}_{i}} \tilde{A}_{i}}\right\|_{L^{2}\left(A\left(1 / 2 \tilde{R}_{i}, 1 / \tilde{R}_{i}\right)\right)}^{\mathrm{dvol}}{ }^{\mathrm{Eurb}} \\
& \approx \tilde{R}_{i}^{2 n-2}\left\|F_{e^{i \tilde{s}_{i}} \tilde{A}_{i}}\right\|_{L^{2}\left(A\left(1 / 2 \tilde{R}_{i}, 1 / \tilde{R}_{i}\right)\right)}^{\mathrm{Euc}} \\
& =\tilde{R}_{i}^{2 n-1}\left\|\sigma_{R}^{*} F_{e^{i \tilde{s}_{i}} \tilde{A}_{i}}\right\|_{L^{2}(A(1 / 2,1))}^{\mathrm{Euc}} \leq c \tilde{R}_{i}^{-n}=c R_{i}^{-1} .
\end{aligned}
$$

The second equality in the above estimate is based on the fact that the Euclidean metric on $\mathbb{P}(1, n)$ lifts to the metric dvol orb on $\tilde{\mathbb{P}}$. For the third equality, we observe that the metric dvol orb is equal to the metric $\tilde{R}_{i}^{-2 n+2} \mathrm{dvol}_{\text {Euc }}$ multiplied by a scalar function that takes values between 1 and $2^{2 n-2}$. We remark that if the annulus were replaced by a ball we would not have an upper bound for that multiplicative factor. Finally, the inequality is the result of applying (36), concluding the proof of Proposition 3.19. 


\section{From VORTICES TO HOLOMORPHIC MAPS}

In this section, we prove Theorem 3.1 (b). The main tool used is the following result on the asymptotic behavior of a finite energy vortex $(A, u)$ on $\mathbb{C}$, which is a slight generalization of a result of Ziltener [52].

Proposition 4.1. (Exponential Decay for Vortices) Suppose the G-action on $X^{\mathrm{ss}}$ has finite stabilizers and the quotient $X / / G$ is compact. Let $n$ be a positive integer such that for any $x \in X^{\mathrm{ss}}$, the order of the stabilizer group $\left|G_{x}\right|$ divides $n$. Let $(A, u)$ be a finite energy vortex on $\mathbb{C} \backslash B_{1}$ with target $X$ whose image is contained in a compact subset of $X$. Then, for every $\epsilon>0$, there is a constant $C$ such that

$$
\left|F_{A}(z)\right|^{2}+\left|\mathrm{d}_{A} u(z)\right|^{2}+|\Phi(u(z))|^{2} \leq C|z|^{-2-\frac{2}{n}+\epsilon}, \quad \forall z \in \mathbb{C} \backslash B_{1} .
$$

The norms are taken with respect to the standard Euclidean metric on $\mathbb{C}$.

Ziltener [52] proves this result for $n=1$, in Section 5, we explain how it generalizes to the case when $X / / G$ is an orbifold. The following is a conclusion of Proposition 4.1. The proofs appear in Section 5.

Proposition 4.2. (Removal of singularity for vortices at infinity) Assume the setting of Proposition 4.1. Further, assume that the restriction of $A$ in radial gauge to the circle $\{|z|=r\} \simeq S^{1}$ is $\mathrm{D}+a \mathrm{~d} \theta$, for $r \geq 1$. For any $p>2$ that satisfies $p<\frac{2}{1-\frac{1}{n}}$ in case $n>1$ and $0<\gamma<\frac{1}{n}-1+\frac{2}{p}$, there exist a constant $c$, a point $x_{0} \in \Phi^{-1}(0)$ and a gauge transformation $k_{0} \in W^{1, p}([0,2 \pi], K)$ such that

$$
\lim _{r \rightarrow \infty} \max _{\theta \in[0,2 \pi]} d\left(x_{0}, k_{0}(\theta) u\left(r e^{i \theta}\right)\right)=0, \quad\left\|k_{0}^{-1} \partial_{\theta} k_{0}+a(r, \cdot)\right\|_{L^{p}([0,2 \pi], K)}<c r^{-\gamma} .
$$

The gauge transformation $k_{0}$ can be chosen so that $k_{0}(0)=\mathrm{Id}$. The point $x_{0}$ is fixed by $k_{0}(2 \pi)$, so $k_{0}(2 \pi)^{n}=\mathrm{Id}$.

\subsection{Proof of Theorem 3.1 (b).}

Proof. (Proof of Theorem $3.1(\mathrm{~b}))$ Let $(A, u)$ be a finite energy affine vortex with bounded image. The singularity of the vortex $(A, u)$ at infinity can be removed in a weak sense via a unitary gauge transformation of class $W^{1, p}$. We work on the chart of the weighted projective line that contains the point at infinity, which is $\tilde{U}_{1} \simeq \mathbb{C}$ in the terminology of (8). The vortex $(A, u)$ lifts to $(\tilde{A}, \tilde{u})(z):=(A, u)\left(z^{-n}\right)$, defined over $\tilde{U}_{1} \backslash\{0\}$. We assume that $A$, and hence $\tilde{A}$ is in radial gauge in a punctured neighborhood of $z=0$ in $\tilde{U}_{1}$. We define a gauge transformation $\tilde{k}_{1}$ on $\tilde{U}_{1} \backslash\{0\}$ so that the pair $\left(\tilde{k}_{1} \tilde{A}, \tilde{k}_{1} \tilde{u}\right)$ extends to a gauged holomorphic map in $\left(L_{\text {loc }}^{p} \times W_{\text {loc }}^{1, p}\right)\left(\tilde{U}_{1}\right)$. Define $\tilde{k}_{1}(r, \theta):=k_{0}(n \theta)$ where $k_{0} \in W^{1, p}([0,2 \pi], K)$ is given by Proposition 4.2. Recall that $k_{0}$ was only defined on $[0,2 \pi]$, which can be extended as $k_{0}(\theta+2 \pi)=$ $k_{0}(2 \pi) k_{0}(\theta)$. This way, $\tilde{k}_{1}$ is a well-defined gauge transformation on $\mathbb{C} \backslash\{0\}$ because $k_{0}(2 \pi)^{n}=\mathrm{Id}$. By Proposition 4.1, $\tilde{k}_{1} \tilde{u}$ extends continuously over $\infty$, with $\tilde{k}_{1} \tilde{u}(\infty)=$ $x_{0}$. The connection $\tilde{k}_{1} \tilde{A}$ is in radial gauge, so $\tilde{A}=\mathrm{D}+a \mathrm{~d} \theta$. Then, using (38),

$$
\left\|\tilde{k}_{1} \tilde{A}\right\|_{L^{p}\left(B_{1}\right)}^{p}=\int_{0}^{1} \int_{0}^{2 \pi}\left|\frac{1}{r}\left(k_{0}^{-1} \partial_{\theta} k_{0}+a\left(r^{-n}, \theta\right)\right)\right|^{p} r \mathrm{~d} \theta d r \leq c \int_{0}^{1} r^{n \gamma p+1-p} d r .
$$


This expression is finite because $2<p<2\left(1+\frac{1}{n}\right)$, so we can choose

$$
\frac{1}{n}\left(1-\frac{2}{p}\right)<\gamma<\frac{1}{n}-1+\frac{2}{p} .
$$

Proposition 4.2 is applicable with these values.

If the gauge transformation $k_{0}$ were smooth, we could have used it to construct a principal bundle over the weighted projective line, on which the given vortex would be $p$-bounded. But since $k_{0}$ is not smooth, we have to take an indirect approach involving complex gauge transformations. We transform the vortex into a smooth gauged holomorphic map on a $K$-bundle over the weighted projective line. Then, we argue that the complex gauge transformation used is $p$-bounded over that bundle.

We now show that there is a complex gauge transformation, called $\tilde{h}$, in a punctured neighborhood of infinity in the weighted projective line, that takes the given vortex to an untwisted holomorphic map in the neighborhood of infinity, i.e. a trivial connection and holomorphic map. For $0<\tilde{R}<1$, let $\sigma_{\tilde{R}}: B_{1} \rightarrow B_{\tilde{R}}$ denote the dilation function $x \mapsto \tilde{R} x$. Writing $\tilde{k}_{1} \tilde{A}=\mathrm{D}+\tilde{a}$, we have $\sigma_{\tilde{R}}^{*}\left(\tilde{k}_{1} \tilde{A}\right)=\mathrm{D}+R \tilde{a}(R \cdot)$. Then,

$$
\left\|\sigma_{R}^{*}\left(\tilde{k}_{1} \tilde{A}\right)\right\|_{L^{p}\left(B_{1}\right)}=\|\tilde{R} \tilde{a}(\tilde{R} \cdot)\|_{L^{p}\left(B_{1}\right)}=\tilde{R}^{1-\frac{2}{p}}\|\tilde{a}\|_{L^{p}\left(B_{\tilde{R}}\right)}
$$

For some $\tilde{R} \in(0,1],\left\|\sigma_{\tilde{R}}^{*} \tilde{A}\right\|_{L^{p}\left(B_{1}\right)}$ is small enough that Lemma 4.3 is applicable. By Lemma 4.3 there is a unique $\tilde{\xi} \in W_{0}^{1, p}\left(B_{\tilde{R}}, \mathfrak{k}\right)$ such that the connection $e^{i \tilde{\xi}} \tilde{k}_{1} \tilde{A}$ is flat. Further, up to left multiplication by a constant, there is a unique gauge transformation $\tilde{k}_{2} \in W^{1, p}\left(B_{\tilde{R}}, K\right)$ such that the connection $\tilde{k}_{2} e^{i \tilde{\xi}} \tilde{k}_{1} \tilde{A}$ is the trivial connection. Choose $\tilde{k}_{2}$ so that $\tilde{k}_{2}(0)=\mathrm{Id}$. Denote by $\tilde{h}:=\tilde{k}_{2} e^{i \tilde{\xi}} \tilde{k}_{1}: B_{\tilde{R}} \backslash\{0\} \rightarrow G$ the complex gauge transformation we have calculated so far. It transforms the map $\tilde{u}$ to a holomorphic map near infinity. This is because $\bar{\partial}(\tilde{h} \tilde{u})=0$ on $B_{\tilde{R}} \backslash\{0\}$ and $\tilde{h} \tilde{u}\left(B_{\tilde{R}}\right)$ extends continuously over 0 . So, we can work on a chart of $X$ and use the removal of singularities for holomorphic functions ([39, Theorem 3.1]) to show that $\tilde{h} \tilde{u}$ extends holomorphically over 0 . Finally, the complex gauge transformation $\tilde{h}$ satisfies the symmetry property $\sigma_{n}^{*}(\tilde{h})=k_{0}(2 \pi) \tilde{h}$. This follows from the symmetry properties of $\tilde{k}_{1} \tilde{A}, \tilde{\xi}$ and $\tilde{k}_{2}$. In particular, we have $\sigma_{n}^{*}\left(\tilde{k}_{1} A\right)=k_{0}(2 \pi) A$. By the uniqueness of $\tilde{\xi}$ and $\tilde{k}_{2}$, we can say that $\tilde{\xi} \circ \sigma_{n}=\operatorname{Ad}_{k_{0}(2 \pi)} \tilde{\xi}$ and $\tilde{k}_{2} \circ \sigma_{n}=k_{0}(2 \pi) \tilde{k}_{2} k_{0}(2 \pi)^{-1}$.

The complex gauge transformation $\tilde{h}$ calculated in the last paragraph will extend over the affine line after it is given a constant twist, and it will transform the given vortex to a gauged holomorphic map that extends smoothly over infinity. Suppose $k_{0}:[0,2 \pi] \rightarrow K$ is homotopic (with fixed end points) to the geodesic $\theta \mapsto e^{-\lambda \theta}$. The complex gauge transformation $e^{n \lambda \theta} \tilde{h}$ is symmetric under the $\mathbb{Z}_{n}$-action, so it descends to a complex gauge transformation $g_{1}$ on $\mathbb{C} \backslash B_{R}$ that satisfies $e^{n \lambda \theta} \tilde{h}(z)=g_{1}\left(\frac{1}{z^{n}}\right)$. On $\mathbb{C} \backslash B_{R}, g_{1} A$ is equal to $\mathrm{D}+\lambda \mathrm{d} \theta$, which is a smooth connection. The map $g_{1} u$ is $\bar{\partial}_{g_{1} A^{-}}$ holomorphic, so it is smooth on $\mathbb{C} \backslash B_{R}$ by elliptic regularity. The complex gauge transformation $g_{1}: \mathbb{C} \backslash B_{R} \rightarrow G$ is smooth because both $u$ and $g_{1} u$ are smooth and $u\left(\mathbb{C} \backslash B_{R}\right) \subset X^{\mathrm{ss}}$, where $G$ acts with finite stabilizers. The map $g_{1}: \mathbb{C} \backslash B_{R} \rightarrow G$ is smoothly homotopic to identity. This is because on $B_{\tilde{R}} \backslash\{0\} \subset \tilde{U}_{1}$, both $\tilde{k}_{1}^{-1} \tilde{k}_{2} e^{i \tilde{\xi}} \tilde{k}_{1}$ 
and $e^{n \lambda \theta} \tilde{k}_{1}$ are continuously homotopic to identity. Therefore, there is a smooth complex gauge transformation $g$ on $\mathbb{C}$ that agrees with $g_{1}$ on $\mathbb{C} \backslash B_{2 R}$. The gauged holomorphic map $g(A, u)$ satisfies the conditions in Proposition 2.4 - i.e. on $\mathbb{C} \backslash B_{2 R}$, $g A=\mathrm{D}+\lambda \mathrm{d} \theta$ and $\lim _{r \rightarrow \infty} e^{-\lambda \theta} g u(r, \theta)=x_{0}$. So, $g(A, u)$ extends to a smooth gauged holomorphic map over $P \rightarrow \mathbb{P}(1, n)$ where the principal $K$-bundle $P$ is described by transition functions $\mu=e^{-2 \pi \lambda}$ and $\tau=e^{n \lambda \theta}$. This indeed proves the existence statement of the theorem: the complex gauge transformation $g$ can be written as $g=e^{i \zeta} k$, where $k \in \mathcal{K}(\mathbb{C})$ and $\zeta: \mathbb{C} \rightarrow \mathfrak{k}$ are smooth. We claim that $e^{-i \zeta} \in \mathcal{G}(P)_{\text {bd }}$, so that $k(A, u)$ extends to a $p$-bounded gauged holomorphic map over $P \rightarrow \mathbb{P}(1, n)$. Let $\tilde{\zeta}: \tilde{U}_{1} \backslash\{0\} \rightarrow \mathfrak{k}$ be defined as $\tilde{\zeta}(z)=\zeta\left(\frac{1}{z^{n}}\right)$ and let $\tilde{k}: \tilde{U}_{1} \backslash\{0\} \rightarrow K$ be equal to $\tilde{k}(z)=k\left(\frac{1}{z^{n}}\right)$. By straightforward calculations, on $B_{\tilde{R} / 2^{n}} \backslash\{0\} \subset \tilde{U}_{1} \backslash\{0\}$, we have

$$
e^{i \tilde{\zeta}}=e^{n \lambda \theta} \tilde{k}_{2} e^{i \tilde{\xi}} \tilde{k}_{2}^{-1} e^{-n \lambda \theta}, \quad \tilde{k}=e^{n \lambda \theta} \tilde{k}_{2} \tilde{k}_{1} .
$$

In the trivialization of $P$ on $\tilde{U}_{1}$, the complex gauge transformation $e^{i \zeta}$ is given by $e^{-n \lambda \theta} e^{i \tilde{\zeta}} e^{n \lambda \theta}: \tilde{U}_{1} \backslash\{0\} \rightarrow K$, which is equal to $\tilde{k}_{2} e^{-i \tilde{\xi}} \tilde{k}_{2}^{-1}$ on $B_{\tilde{R} / 2^{n}} \backslash\{0\} \subset \tilde{U}_{1} \backslash\{0\}$. By recalling details from the previous paragraph, $\tilde{k}_{2} e^{-i \tilde{\xi}} \tilde{k}_{2}^{-1}$ extends to a $W^{1, p}$ map on $B_{\tilde{R} / 2^{n}}$. Therefore $g$ is in $\mathcal{G}(P)_{\text {bd }}$ and the pair $k(A, u)$ extends to a $p$-bounded gauged holomorphic map over $P$. Hence, the pair $(A, u)$ extends to a $p$-bounded gauged holomorphic map over a bundle that is isomorphic to $P$ (see (10)).

Finally we show uniqueness. Given a finite energy vortex $(A, u)$, the bundle $P \rightarrow$ $\mathbb{P}(1, n)$ is determined uniquely by the path $\left\{\left[0, \frac{2 \pi}{n}\right] \ni \theta \mapsto k_{0}(\theta)\right\}$ up to homotopy and the action of $\operatorname{Ad}_{k}$ for $k \in K$ (see Remark 2.3). The path $k_{0}$ is determined using Proposition 4.2, and the choice of this equivalence class is unique because it has to satisfy the condition

$$
\lim _{r \rightarrow \infty} \max _{\theta \in[0,2 \pi]} d\left(x_{0}, k_{0}(\theta) u\left(r e^{i \theta}\right)\right)=0, \quad \text { where } x_{0}=\lim _{r \rightarrow \infty} u(r, 0) \in \Phi^{-1}(0) .
$$

Now suppose $g_{1}, g_{2} \in \mathcal{G}(P)_{\text {bd }}$ so that $g_{i}(A, u)$ is a smooth gauged holomorphic map on $P$. On $B_{\tilde{R}} \subset \tilde{U}_{1}, g_{1} u, g_{2} u$ are smooth maps to $X^{\text {ss }}$, where $G$ acts with finite stabilizers. So, $g_{1}^{-1} g_{2}$ is smooth in this region and hence $g_{1}^{-1} g_{2} \in \mathcal{G}(P)$. This finishes the proof of Theorem 3.1 (b).

The following Lemma is used in the proof of Theorem 3.1 (b). It says that on a trivial principal bundle, an $L^{p}$-small connection can be transformed to a flat connection via a $W^{1, p}$-small complex gauge transformation.

Lemma 4.3. Let $p>2$ and $\Sigma$ be a compact connected Riemann surface with metric with non-empty boundary. Let $P:=\Sigma \times K$ be the trivial principal $K$-bundle on $\Sigma$. Let $\mathrm{D}$ be the trivial connection on $P$. There are constants $c_{1}, c_{2}$ and $c_{2}^{\prime}$ so that the following holds. Let $A=\mathrm{D}+a$ be a connection on $P$ so that $a \in \Omega^{1}(\Sigma, \mathfrak{k})_{L^{p}}$. If $\|a\|_{L^{p}(\Sigma)}<c_{1}$, there is a unique $\xi \in W^{1, p}(\Sigma, \mathfrak{k})$ satisfying $\left.\xi\right|_{\partial \Sigma}=0, F_{e^{i \xi} A}=0$ and $\|\xi\|_{W^{1, p}} \leq c_{2}\left\|F_{A}\right\|_{W^{-1, p}} \leq c_{2}^{\prime}\|a\|_{L^{p}}$.

Further, on any contractible closed set $\Sigma^{\prime} \subset$ int $\Sigma$, there is a gauge transformation $k \in W^{1, p}\left(\Sigma^{\prime}, K\right)$ so that $k e^{i \xi} A=\mathrm{D}$ on $\Sigma^{\prime}$. The gauge transformation $k$ is unique up to left multiplication by a constant element in $K$. 
On a compact connected Riemann surface $\Sigma$ with non-empty boundary, if the Lie group $K$ is connected, then any principal $K$-bundle $P \rightarrow \Sigma$ is trivializable. Hence it suffices to consider only trivial bundles in Lemma 4.3.

Proof. (Proof of Lemma 4.3) The proof is by an application of the implicit function theorem on the map

$$
\mathcal{F}^{A}: W_{0}^{1, p}(\Sigma, \mathfrak{k}) \rightarrow W^{-1, p}(\Sigma, \mathfrak{k}), \quad \xi \mapsto * F_{e^{i \xi}} .
$$

By (3) and (6), the linearization at $\xi$ is

$$
D \mathcal{F}_{\xi}^{A}=\mathrm{d}_{e^{i \xi} A}^{*} \mathrm{~d}_{e^{i \xi} A}: W_{0}^{1, p}(\Sigma, \mathfrak{k}) \rightarrow W^{-1, p}(\Sigma, \mathfrak{k}) .
$$

In this proof, the constant $c$ indicates a constant that is independent of the connection $A$, whose value varies across appearances.

STEP 1: There is a constant $c_{1}$ such that if a connection $A=\mathrm{D}+$ a satisfies $\|a\|_{L^{p}}<$ $c_{1}$, then $D \mathcal{F}^{A}(0)$ is invertible and the inverse has norm independent of a.

The operator $\mathrm{d}^{*} \mathrm{~d}: W_{0}^{1, p}(\Sigma, \mathfrak{k}) \rightarrow W^{-1, p}(\Sigma, \mathfrak{k})$ is an isomorphism (see appendix D in [45]). Further,

$$
\left(\mathrm{d}_{A}^{*} \mathrm{~d}_{A}-\mathrm{d}^{*} \mathrm{~d}\right) \eta=*[a \wedge * \mathrm{~d} \eta]+\mathrm{d}^{*}[a, \eta]+*[a \wedge *[a \wedge \eta]] .
$$

Using Sobolev multiplication (Proposition 6.2) and by assuming $c_{1}<1$, we have

$$
\left\|\left(\mathrm{d}_{A}^{*} \mathrm{~d}_{A}-\mathrm{d}^{*} \mathrm{~d}\right) \eta\right\|_{W^{-1, p}} \leq c\|\eta\|_{W^{1, p}}\left(\|a\|_{L^{p}}+\|a\|_{L^{p}}^{2}\right) \leq c\|\eta\|_{W^{1, p}}\|a\|_{L^{p}}
$$

so the operator norm satisfies $\left\|\left(\mathrm{d}_{A}^{*} \mathrm{~d}_{A}-\mathrm{d}^{*} \mathrm{~d}\right)\right\| \leq c\|a\|_{L^{p}}$. If this quantity is small enough, by a Neumann series argument, $\mathrm{d}_{A}^{*} \mathrm{~d}_{A}$ is invertible with bounded norm. Let $\Theta:=\left(d_{A}^{*} d_{A}-d^{*} d\right)\left(d^{*} d\right)^{-1}$. If $\|\Theta\|<1$, then the operator Id $+\Theta$ is invertible and the inverse has a bound

$$
\left\|(\mathrm{Id}+\Theta)^{-1}\right\| \leq \sum_{i=0}^{\infty}\|\Theta\|^{i}
$$

Further,

$$
\left(\mathrm{d}_{A}^{*} \mathrm{~d}_{A}\right)^{-1}=\left(\mathrm{d}^{*} \mathrm{~d}\right)^{-1}(\mathrm{Id}+\Theta)^{-1} .
$$

We can choose $c_{1}$ such that if $\|a\|_{L^{p}}<c_{1}$, then,

$$
\left\|\left(\mathrm{d}_{A}^{*} \mathrm{~d}_{A}\right)^{-1}\right\| \leq 2\left\|\left(\mathrm{~d}^{*} \mathrm{~d}\right)^{-1}\right\| .
$$

In the statement of the implicit function theorem (Proposition 6.1), we take $C:=$ $2\left\|\left(\mathrm{~d}^{*} \mathrm{~d}\right)^{-1}\right\|$.

STEP 2: Given a constant $c_{1}$, there is a constant $c$ such that for any connection $A=\mathrm{D}+a$ satisfying $\|a\|_{L^{p}}<c_{1}$ and $\|\xi\|_{W^{1, p}}<1$,

$$
\left\|D \mathcal{F}_{\xi}^{A}-D \mathcal{F}_{0}^{A}\right\|<c\|\xi\|_{W^{1, p}} .
$$

Write

$$
D \mathcal{F}_{\xi}^{A}-D \mathcal{F}_{0}^{A}=\left(\mathrm{d}_{(\exp i \xi) A}^{*} \mathrm{~d}_{(\exp i \xi) A}-\mathrm{d}_{A}^{*} \mathrm{~d}_{A}\right)
$$


Let $(\exp i \xi) A=A+\alpha$. Then,

$$
\left(\mathrm{d}_{(\exp i \xi) A}^{*} \mathrm{~d}_{(\exp i \xi) A}-\mathrm{d}_{A}^{*} \mathrm{~d}_{A}\right) \xi_{1}=*\left[\alpha \wedge * \mathrm{~d}_{A} \xi_{1}\right]+\mathrm{d}_{A}^{*}\left[\alpha \wedge \xi_{1}\right]+*\left[\alpha \wedge *\left[\alpha \wedge \xi_{1}\right]\right] .
$$

As in Step 1, the operator norm of $D \mathcal{F}_{\xi}-D \mathcal{F}_{0}$ is bounded as

$$
\left\|\mathrm{d}_{(\exp i \xi) A}^{*} \mathrm{~d}_{(\exp i \xi) A}-\mathrm{d}_{A}^{*} \mathrm{~d}_{A}\right\| \leq c\left(\|\alpha\|_{L^{p}}+\|\alpha\|_{L^{p}}^{2}\right) \leq c\|\xi\|_{W^{1, p}},
$$

where the last inequality is by Lemma 6.4 and the fact that $\|\xi\|_{W^{1, p}}<1$. If $\|a\|_{L^{p}}<$ $c_{1}$, the constant $c$ is independent of $a$ and $\xi$.

STEP 3: Finishing the proof.

By the result in Step 2, there is a constant $\delta_{0}>0$ such that

$$
\|\xi\|_{W^{1, p}}<\delta_{0} \Longrightarrow\left\|D \mathcal{F}_{\xi}^{A}-D \mathcal{F}_{0}^{A}\right\| \leq \frac{1}{2 C}
$$

The function

$$
(\xi, \Delta \xi) \mapsto D \mathcal{F}^{A}(\xi) \Delta \xi: W_{0}^{1, p}(\Sigma, \mathfrak{k}) \times W_{0}^{1, p}(\Sigma, \mathfrak{k}) \rightarrow W^{-1, p}(\Sigma, \mathfrak{k})
$$

is continuous using the identity (40), Lemma 6.4 and Sobolev multiplication (Proposition 6.2). So $\mathcal{F}^{A}$ is differentiable and the implicit function theorem is applicable. The constant $c_{1}$ can be chosen such that $\left\|F_{A}\right\|_{-1, p}<\frac{\delta_{0}}{8 C}$. This is because by the multiplication theorem,

$$
\left\|F_{A}\right\|_{-1, p} \leq c\left(\|a\|_{L^{p}}+\|a\|_{L^{p}}^{2}\right)<c c_{1} .
$$

Finally, apply Proposition 6.1 with $\delta:=8 C\left\|F_{A}\right\|_{-1, p}$. This ensures $\left\|\mathcal{F}^{A}(0)\right\|=$ $\left\|F_{A}\right\|_{-1, p}<\frac{\delta}{4 C}$ and we get a solution $\xi \in B_{\delta}\left(W^{1, p}\right)$ for $\mathcal{F}^{A}(\xi)=0$. The solution $\xi$ also satisfies $\|\xi\|_{1, p}<8 C\|F(A)\|_{-1, p}<c\|a\|_{L^{p}}$. The constants in this inequality are suitable values for $c_{2}$ and $c_{2}^{\prime}$ in the statement of the Lemma.

We next prove the second statement of the lemma. We use the fact that a flat $L^{p}$ connection is gauge-equivalent to a connection that is smooth away from the boundary $\partial \Sigma$. Indeed, this is proved in [44, Theorem 3.1] for a base manifold without boundary, but since we only want interior regularity, the proof is identical. We briefly outline this proof: For an $L^{p}$ connection $A$, if we choose a smooth connection $\tilde{A}_{0}$ close enough to $A$ (in the $L^{p}$ norm), there is a gauge transformation $k \in W^{1, p}$ that puts $A$ in coulomb gauge with respect to $\tilde{A}_{0}$, that is, if $a:=k A-\tilde{A}_{0}$,

$$
\mathrm{d}_{\tilde{A}_{0}}^{*} a=0 .
$$

This is the content of the local slice theorem (Theorem 8.3) in [45]. Now, one has control over $\mathrm{d} a$ and $\mathrm{d}^{*} a$, using elliptic bootstrapping as in [44], it can be shown that $k A$ is smooth away from $\partial \Sigma$. Similar to the first statement in the lemma, here we rely on the ellipticity of $\Delta: W_{0}^{1, p} \rightarrow W^{-1, p}$.

Finally note that a smooth flat connection on a contractible set is gauge equivalent to the trivial connection. The uniqueness statement in the lemma is obvious because the trivial connection is preserved only by constant gauge transformations. This finishes the proof of Lemma 4.3 
Remark 4.4. Lemma 4.3 is true for higher regularity connections. Suppose $k \in \mathbb{Z}_{\geq 0}$, $p>1$ such that $(k+1) p>2$. If $a \in W^{k, p}$, then $\xi \in W^{k+1, p}$ can be produced satisfying the conditions of Lemma 4.3. The proof of the higher regularity statement is analogous and is more standard.

4.2. Unique affine vortex in a complex gauge orbit. The following is the last component in the proof of the main Theorem 3.1. To state it, we assume the result of part (b) of this theorem.

Proposition 4.5. (At most one vortex in a complex gauge orbit) Suppose $\left(A_{0}, u_{0}\right)$ and $\left(A_{1}, u_{1}\right)$ are finite energy vortices on $\mathbb{C}$ that extend to a p-bounded gauged holomorphic map over a bundle $P \rightarrow \mathbb{P}(1, n)$. Suppose further that they are related by a complex gauge transformation $g \in \mathcal{G}(P)_{\mathrm{bd}}$. Then $g$ is a unitary gauge transformation, i.e. $g \in \mathcal{K}(P)_{\mathrm{bd}}$.

Proof. The proof proceeds in the same way as the corresponding result for vortices on a compact Riemann surface (Proposition 3.6). The complex gauge transformation $g$ can be written as $g=k e^{i \xi}$, where $k \in \mathcal{K}(P)_{\text {bd }}$ and $\xi \in \Gamma(\mathbb{P}(1, n), P(\mathfrak{k}))_{\text {bd }}$. We can assume $k=\operatorname{Id}$ and $\left(A_{1}, u_{1}\right)=e^{i \xi}\left(A_{0}, u_{0}\right)$. Let $\left(A_{t}, u_{t}\right):=e^{i t \xi}(A, u)$ for $t \in[0,1]$. We write

$$
\begin{aligned}
\frac{d}{d t} \int_{\mathbb{C}}\left\langle * F_{A_{t}, u_{t}}, \xi\right\rangle & =\int_{\mathbb{C}}\left\langle\mathrm{d}_{A_{t}}^{*} \mathrm{~d}_{A_{t}} \xi+u_{t}^{*} d \Phi\left(J(\xi)_{u_{t}}\right), \xi\right\rangle_{\mathfrak{k}} \\
& =\left\|\mathrm{d}_{A_{t}} \xi\right\|_{L^{2}}^{2}+\int_{\mathbb{C}} \omega_{u_{t}}\left((\xi)_{u_{t}}, J(\xi)_{u_{t}}\right)+\lim _{r \rightarrow \infty} \int_{\partial B_{r}}\left\langle\nabla_{A_{t}, \nu} \xi, \xi\right\rangle_{\mathfrak{k}}
\end{aligned}
$$

where $\nabla_{A_{t}, \nu} \xi$, is the covariant derivative of $\xi$ along $\nu$, the outward normal unit vector field to $\partial B_{r}$.

However, for the above computation to make sense, we need to show that the terms $\left\|\mathrm{d}_{A_{t}} \xi\right\|_{L^{2}(\mathbb{C})}$ and $\int_{\mathbb{C}} \omega_{u_{t}}\left((\xi)_{u_{t}}, J(\xi)_{u_{t}}\right)$ are finite and the boundary term vanishes.

STEP 1: $\left\|\mathrm{d}_{A_{t}} \xi\right\|_{L^{2}}<\infty$ for $t \in[0,1]$.

We work on the chart containing infinity - this is $\tilde{U}_{1}$ from (8). Consider $B_{\tilde{R}} \subset \tilde{U}_{1}$, for some $\tilde{R}>0$. We have $A_{0} \in L^{p}\left(B_{\tilde{R}}\right)$ and $\xi \in W^{1, p}\left(B_{\tilde{R}}\right)$. Using Lemma 6.4 for the action of the complex gauge transformation $e^{i t \xi}$ on the connection $A_{0}$, we can say $A_{t}-A_{0} \in L^{p}\left(B_{\tilde{R}}\right)$. Since $p>2,\left\|\mathrm{~d}_{A_{t}} \xi\right\|_{L^{2}\left(B_{\tilde{R}}\right)}$ is finite. Let $R=(\tilde{R})^{-1 / n}$. By conformal invariance of the $L^{2}$ norm of one-forms, the norms $\left\|\mathrm{d}_{A_{t}} \xi\right\|_{L^{2}\left(\mathbb{C} \backslash B_{R}\right)}$ and $\left\|\mathrm{d}_{A_{t}} \xi\right\|_{L^{2}\left(B_{\tilde{R}}\right)}$ are equal, so both are finite, and hence $\left\|\mathrm{d}_{A_{t}} \xi\right\|_{L^{2}}<\infty$ for $0 \leq t \leq 1$.

STEP 2: $\int_{\mathbb{C}} \omega_{u_{t}}\left((\xi)_{u_{t}}, J(\xi)_{u_{t}}\right)<\infty$ for $t \in[0,1]$.

We use asymptotic decay of vortices (Proposition 4.1) to obtain an asymptotic bound on $|\xi|$ : fix an $0<\epsilon<\frac{2}{n}$, let $\delta=\frac{2}{n}-\epsilon$, then for an affine vortex $(A, u)$ there is a constant $c$ so that for $z \in \mathbb{C} \backslash B_{1}$,

$$
e_{A, u}(z) \leq c|z|^{-2-\delta} .
$$

Therefore, $\left|\Phi\left(u_{0}(z)\right)\right|,\left|\Phi\left(u_{1}(z)\right)\right| \leq c|z|^{-1-\frac{\delta}{2}}$. 
We prove a similar asymptotic bound on $\xi$ also. For any $c>0$, define $Z_{c}:=$ $\{|\Phi|<c\} \subset X$. For any $x \in X$, define

$$
\Psi_{x}: \mathfrak{k} \rightarrow \mathfrak{k}, \quad s \mapsto \Phi\left(e^{i s} x\right)-\Phi(x) .
$$

For all $x \in \Phi^{-1}(0)$, the derivative $d \Psi_{x}(0)$ is invertible. Since $\Phi$ is proper, we can choose $\epsilon_{1}>0$ such that $d \Psi_{x}(0)$ is invertible for all $x \in Z_{\epsilon_{1}}$. Define

$$
\Psi: Z_{\epsilon_{1}} \times \mathfrak{k} \rightarrow Z_{\epsilon_{1}} \times \mathfrak{k} \quad(z, s) \mapsto\left(z, \Psi_{z}(s)\right)=\left(z, \Phi\left(e^{i s} z\right)-\Phi(z)\right) .
$$

For all $z \in Z_{\epsilon_{1}}$, the map $d \Psi(z, 0)$ is an isomorphism, so $\Psi$ is locally an isomorphism. That is, there is an open neighborhood of $U$ of $(z, 0)$, such that $\Psi: U \rightarrow f(U)$ is a diffeomorphism. By the compactness of $\bar{Z}_{\epsilon_{1}}$, there exists $\epsilon_{2}>0$ such that $\Psi$ has a smooth inverse on $Z_{\epsilon_{1}} \times B_{\epsilon_{2}}(\mathfrak{k})$. Set $\epsilon:=\min \left\{\epsilon_{1}, \epsilon_{2}\right\}$. We can conclude that there is a constant $c$ such that for $x, y \in Z_{\epsilon}$ satisfying $y=e^{i s} x$ for some $s \in \mathfrak{k}$,

$$
|s|<c|\Phi(x)-\Phi(y)| \text {. }
$$

We can choose $0<\epsilon_{2}^{\prime}<\epsilon_{2}$ such that for all $z \in Z_{\epsilon_{1}}$, the straight line connecting 0 and any point in $\Psi_{z}^{-1}\left(B_{\epsilon_{2}^{\prime}}\right)$ is contained in $\Psi_{z}^{-1}\left(B_{\epsilon_{2}}\right)$. Set $\epsilon^{\prime}:=\min \left\{\epsilon_{1}, \epsilon_{2}^{\prime}\right\}$. For any $x, y \in Z_{\epsilon^{\prime}}$ satisfying $y=e^{i s} x$ for some $s \in \mathfrak{k}$, the curve $\left\{e^{i t s} x: t \in[0,1]\right\}$ is contained in $Z_{\epsilon}$.

Let $R>0$ be large enough that both $u_{0}, u_{1}$ map $\mathbb{C} \backslash B_{R}$ to $Z_{\epsilon^{\prime}}$. Recall $u_{1}=e^{i \xi} u_{0}$. By the preceding discussion, $u_{t}\left(\mathbb{C} \backslash B_{R}\right) \subset Z_{\epsilon}$ for all $t \in[0,1]$ and by (42),

$$
|\xi(x)|<c\left|\Phi\left(u_{1}(x)\right)-\Phi\left(u_{0}(x)\right)\right|<c|z|^{-1-\frac{\delta}{2}}
$$

for $x \in \mathbb{C} \backslash B_{R}$. For $z \in Z_{\epsilon}$, the operator $L_{z}$ (see (16)) is uniformly bounded. So, there is a constant $c$ so that

$$
\left|\omega_{u_{t}}\left((\xi)_{u_{t}}, J(\xi)_{u_{t}}\right)(z)\right|<c|z|^{-2-\delta}
$$

for $z \in \mathbb{C} \backslash B_{R}$ and hence,

$$
\int_{\mathbb{C}} \omega_{u_{t}}\left((\xi)_{u_{t}}, J(\xi)_{u_{t}}\right)<\infty, \quad 0 \leq t \leq 1
$$

STEP 3: $\lim _{r \rightarrow \infty} \int_{\partial B_{r}}\left\langle\nabla_{A_{t}, \nu} \xi, \xi\right\rangle_{\mathfrak{k}}=0$ for $t \in[0,1]$.

In step 1 , we showed that $A_{t}$ is in $L^{2}\left(\mathbb{C} \backslash B_{R}\right)$, but we do not know anything yet about the restriction of $A_{t}$ to the boundary $\partial B_{R}$. We now obtain a $C^{0}$-bound under suitable local trivializations of $P$. For this we cover $\mathbb{C} \backslash B_{R}$ by identical open sets. Let $S \subseteq \mathbb{C}$ be an open set with smooth boundary such that

$$
\left[-\frac{3}{4}, \frac{3}{4}\right] \times\left[-\frac{3}{4}, \frac{3}{4}\right] \subseteq S \subseteq[-1,1] \times[-1,1] .
$$

Then, $\{S+(x, y):|x|,|y| \geq R-2\}$ is a cover of $\mathbb{C} \backslash B_{R}$. Let $S^{\prime \prime} \Subset S^{\prime} \Subset S$ be such that their translates (by integers) also cover $\mathbb{C} \backslash B_{R}$. The quantity $\left\langle\mathrm{d}_{A_{t}} \xi, \xi\right\rangle_{\mathfrak{k}} \in \Omega^{1}(\Sigma)$ is gauge-invariant, so on each $S+(x, y)$, we can choose a different trivialization to study it. We focus on a single set $S_{x y}:=S+(x, y)$ and let $r:=\sqrt{x^{2}+y^{2}}$. In the 
following discussion, the constant $c$ is independent of $(x, y)$ and $r$. Fix a trivialization of $\left.P\right|_{S_{x y}}$ so that $A_{0}=\mathrm{D}+a_{0}$ is in Uhlenbeck gauge i.e.

$$
\left\|a_{0}\right\|_{W^{1, p}\left(S_{x y}\right)}<c\left\|F\left(A_{0}\right)\right\|_{L^{p}\left(S_{x y}\right)}<c .
$$

Under this trivialization, we write $A_{t}=\mathrm{D}+a_{t}$. By applying a gauge transformation $k: S_{x y} \rightarrow K$, we can put $A_{1}$ in Uhlenbeck gauge - i.e. if $k A_{1}=\mathrm{D}+\tilde{a}_{1}$ then,

$$
\left\|\tilde{a}_{1}\right\|_{W^{1, p}\left(S_{x y}\right)}<c\left\|F\left(A_{1}\right)\right\|_{L^{p}\left(S_{x y}\right)}<c .
$$

Denote $g=k e^{i \xi}$, so $g A_{0}=\mathrm{D}+\tilde{a}_{1}$. As in the proof of Lemma 3.18, we can write $a^{0,1} g=-\bar{\partial}_{A_{0}} g$, where $a=\tilde{a}_{1}-a_{0}$. By elliptic regularity,

$$
\begin{aligned}
\|g\|_{W^{1, p}\left(S_{x y}^{\prime}\right)} & \leq c\left(\|\bar{\partial} g\|_{L^{p}\left(S_{x y}\right)}+\|g\|_{L^{p}\left(S_{x y}\right)}\right) \\
& \left.\leq c\left(\left\|a^{0,1} g\right\|_{L^{p}\left(S_{x y}\right)}+\left\|a_{0}^{0,1} g\right\|_{L^{p}\left(S_{x y}\right)}\right)+c\|g\|_{L^{\infty}\left(S_{x y}\right)}\right)
\end{aligned}
$$

There is an $L^{\infty}$ bound on $g$ from (43) and the fact that $K$ is compact. Together with the bounds on $a$ and $a_{0}$ (from (44) and (45)), this shows that $\|g\|_{W^{1, p}\left(S_{x y}^{\prime}\right)} \leq c$. By applying elliptic regularity again, we can show $\|g\|_{W^{2, p}\left(S_{x y}^{\prime \prime}\right)} \leq c$. Since (4) is a diffeomorphism,

$$
\|\xi\|_{W^{2, p}\left(S_{x y}^{\prime \prime}\right)} \leq c .
$$

By Lemma 6.4, we get

$$
\left\|a_{t}-a_{0}\right\|_{W^{1, p}\left(S_{x y}^{\prime \prime}\right)}<c
$$

Together with the bound on $a_{0}$, this uniformly bounds $\left\|a_{t}\right\|_{W^{1, p}\left(S_{x y}\right)}$. By Sobolev embedding

$$
\left\|a_{t}\right\|_{C^{0}\left(S_{x y}\right)}<c
$$

for $0 \leq t \leq 1$.

Consider the integral $\int_{\partial B_{r}}\left\langle\nabla_{A_{t}, \nu} \xi, \xi\right\rangle_{\mathfrak{k}}$. We partition the curve into segments, so that each segment lies in a single set $S_{x y}^{\prime \prime}$. Write $\nabla_{A_{t}, \nu} \xi=\frac{\partial}{\partial \nu} \xi+\left[\left(a_{t}\right)_{\nu}, \xi\right]$. We have $C^{0}$ bounds on $\frac{\partial}{\partial \nu} \xi$ and $\left(a_{t}\right)_{\nu}$ using (46) and (47). Together with the asymptotic bound on $\xi(43)$, the result of Step 3 is proved.

STEP 4: Finishing the proof.

We have shown that the equation (41) holds and the boundary term vanishes as $r \rightarrow \infty$. So,

$$
\frac{d}{d t} \int_{\mathbb{C}}\left\langle * F_{A_{t}, u_{t}}, \xi\right\rangle>0
$$

if $\xi \neq 0$. Since $F_{A_{0}, u_{0}}=F_{A_{1}, u_{1}}=0$, this implies that $\xi=0$, proving Proposition 4.5 .

Proof. (Proof of Proposition 1.2) We first consider the case of a line bundle $X=\mathbb{C}$ on which $G$ acts with weight $\nu \in \mathfrak{g}^{\vee}$. Suppose $P$ is a principal bundle with $[P]=d$. Then the associated orbifold line bundle $P(X)$ has degree (pairing of the first Chern class with the rational fundamental class) $\langle\nu, d\rangle \in \mathbb{Q}$. By our set-up $\langle\nu, d\rangle=\frac{m}{n}$ for some $m \in \mathbb{Z}$. Up to isomorphism, there is a unique holomorphic line bundle 
on $\mathbb{P}(1, n)$ of this degree [38, Theorem 5.1], this line bundle is described as: $L=$ $\left(\tilde{U}_{1} \times \mathbb{C}\right) \sqcup\left(U_{2} \times \mathbb{C}\right) / \sim$ where $\sim$ is as follows:

$$
\begin{aligned}
& (z, l) \sim\left(\sigma_{n} z, e^{-2 \pi i m / n} l\right) \quad(z, l) \in \tilde{U}_{1} \times \mathbb{C} \\
& (z, l) \sim\left(w, z^{m} l\right) \quad 0 \neq z \in \tilde{U}_{1}, w \in U_{2}, w=\frac{1}{z^{n}}, \in \mathbb{C}
\end{aligned}
$$

In the framework of Section 2.5, the transition functions $\mu: \tilde{U}_{1} \rightarrow \mathbb{C}^{\times}$and $\tau$ : $\tilde{U}_{1} \backslash\{0\} \rightarrow \mathbb{C}^{\times}$are holomorphic and are $\mu(z):=e^{-2 \pi i m / n}$ and $\tau(z)=z^{m}$. Holomorphic sections on $u: \mathbb{P}(1, n) \rightarrow P(X)$ are of the following form:

$$
U_{2} \ni w \mapsto u_{0}+u_{1} w+\cdots+u_{q} w^{q}, \quad \tilde{U}_{1} \ni z \mapsto z^{m}\left(u_{0}+u_{1} z^{-n}+\cdots+u_{q} z^{-q n}\right),
$$

where $q=\lfloor m / n\rfloor$. It is clear that $u(\infty)=0$ if $m / n$ is not an integer, and otherwise $u(\infty)=u_{q}=u^{(q)} / q$ ! . For the case of a vector bundle $X=\mathbb{C}^{k}$, the expression for $u_{\infty}$ can be derived in a similar way. Using Theorem 3.1, the section $u$ is complex gauge equivalent to an affine vortex if and only if $u(\infty) \in X^{\mathrm{ss}}$.

We next show when two such sections are complex gauge equivalent. Consider a holomorphic complex gauge transformation $g \in \mathcal{G}(P)$ relating sections $u_{1}, u_{2}$. The complex gauge transformation $g$ induces an isomorphism on each line bundle $P \times{ }_{\nu_{i}} \mathbb{C}$, which we call $\nu_{i}(g)$. Since the group is Abelian, $\nu_{i}(g)$ is a holomorphic map from $\mathbb{P}(1, n)$ to $\mathbb{C}^{\times}$, which is necessarily a constant. Since the weights $\nu_{1}, \ldots, \nu_{k} \operatorname{span} \mathfrak{g}^{\vee}$, we conclude $g: \mathbb{P}(1, n) \rightarrow G$ is a constant.

\section{Asymptotic DECAY FOR VORTICES}

Proposition 4.1 is a consequence of a decay result for vortices on a cylinder (Proposition 5.3) and a result about the limit behavior of $u$ as $z \rightarrow \infty$ (Proposition 5.4).

Definition 5.1. (Energy density) Let $U \subset \mathbb{C}$ be an open subset. Suppose $\lambda: U \rightarrow$ $\mathbb{R}_{\geq 0}$ is a smooth function. Let $(A, u)$ be a vortex from the principal bundle $P \rightarrow U$ to $X$ with respect to the metric $\omega=\lambda^{2} d s \wedge d t$. The energy density of $(A, u)$ is

$$
e_{(A, u)}(z):=\frac{1}{2}\left(\left|F_{A}(z)\right|^{2}+\left|\mathrm{d}_{A} u(z)\right|^{2}+|\Phi(u(z))|^{2}\right)
$$

for any $z \in U$. The norms are defined as follows : for any form $\alpha \in \Omega^{*}(U, \mathfrak{k})$, if $\alpha \wedge *_{\lambda} \alpha=f d s \wedge d t$, then $|\alpha(z)|^{2}=|f|^{2}$. Here, $*_{\lambda}$ denotes the Hodge star with respect to the metric $\lambda^{2} d s \wedge d t$.

Definition 5.2. (Admissible metric) Let $a>0$. On a half cylinder $\Sigma:=\{(s, t)$ : $s \geq 0, t \in \mathbb{R} / a \mathbb{Z}\}$, the metric $\omega_{\Sigma}=\lambda^{2} d s \wedge d t$ is admissible if

$$
\lambda \geq \frac{2 \pi}{a m_{\Phi^{-1}(0)}} \sup _{\Sigma}\left(\Delta\left(\lambda^{-2}\right)+\left|d\left(\lambda^{-1}\right)\right|^{2}\right)<2 m_{\Phi^{-1}(0)}^{2}
$$

where $m_{\Phi^{-1}(0)}=\inf _{x \in \Phi^{-1}(0)}\left|L_{x}\right|$, where $L_{x}$ is defined by (16).

Proposition 5.3. (Decay for vortices on the half cylinder, [52, Theorem 1.3]) Let $\Sigma$ be a half cylinder

$$
\Sigma:=\{(s, t): s \geq 0, t \in \mathbb{R} / a \mathbb{Z}\}
$$


with an admissible metric $\omega_{\Sigma}=\lambda^{2} d s \wedge d t$ where $\lambda=\lambda(s, t)$ is a positive function. Suppose $G$ acts freely on $X^{\mathrm{ss}}$ and $(A, u)$ is a finite energy vortex from the trivial bundle $\Sigma \times K$ to $X$ such that $\overline{u(\Sigma)}$ is compact. Then, for every $\epsilon>0$, there is a constant $C$ such that

$$
e_{(A, u)}(s+i t) \leq C \lambda^{-2} e^{\left(-\frac{4 \pi}{a}+\epsilon\right) s} .
$$

Proposition 5.4. Suppose $(A, u)$ is a finite energy vortex with bounded image on the half cylinder $\Sigma$ (described in Proposition 5.3). Let $\pi_{G}: X^{\mathrm{ss}} \rightarrow X / / G$ denote the projection. Then, $\lim _{s \rightarrow \infty} \pi_{G} \circ u(s, t)$ exists.

Proof. The proof uses a combination of results from Ziltener [52] and McDuffSalamon [29] and extends them to the case of orbifold quotient/target. For the mean value inequality, we use Lemma 3.3 in [52]. The proof of this result works when the action of $K$ on $\Phi^{-1}(0)$ has finite stabilizers. In the setting of Proposition 5.3 , this gives that there exists a number $s_{0} \geq \frac{1}{2}$ such that for $z=(s, t)$ with $s \geq s_{0}$,

$$
e_{(A, u)}(z) \leq \frac{32}{\pi} E\left((A, u) ; B_{\frac{1}{2}}(z)\right) \text {. }
$$

Since $E((A, u) ; \Sigma)$ is finite, the right side goes to 0 as $s \rightarrow \infty$. So, for large enough $s_{0}, \Phi(u(s, t))$ is close enough to 0 and so $u(s, t) \in X^{\mathrm{ss}}$. Furthermore, $u_{G}:=\pi_{G} \circ u$ is well-defined and is a holomorphic map to $X / / G$. Also,

$$
\begin{aligned}
\ell\left(u_{G}\left(\left\{s=s_{1}, t \in \mathbb{R} / a \mathbb{Z}\right\}\right)\right) & \leq \int_{0}^{a}\left|d u_{G}\left(s_{1}, t\right)\right| d t \\
& \leq \int_{0}^{a}\left|\mathrm{~d}_{A} u\left(s_{1}, t\right)\right| d t \rightarrow 0 \quad \text { as } s_{1} \rightarrow \infty .
\end{aligned}
$$

Now, we switch to working on $X / / G$ to prove the result. For every $p \in X / / G$, there is a neighborhood $U_{p}$ and a uniformizing chart $\left(V_{p}, G_{p}, \pi\right)$ such that $V_{p} \subset \mathbb{C}^{N}$, $G_{p}$ is a finite group acting on $U_{p}$ and $\pi: U_{p} \rightarrow V_{p}$ induces a homeomorphism from $U_{p} / G_{p}$ to $V_{p}$ (see [9]). Each $U_{p}$ has a $G_{p}$-invariant symplectic form that descends to the symplectic form on $X / / G$. The quotient $X / / G$ is compact, so it can be covered by a finite number of such neighborhoods $U_{1}, \ldots, U_{k}$. Fix a constant $\delta_{0}>0$ such that for any $p \in X / / G, B_{p}\left(\delta_{0}\right) \subset U_{i}$ for some $i \in\{1, \ldots, k\}$. If the length of the loop $\gamma: S^{1} \rightarrow X / / G$ is less than $\delta_{0}$, it can be lifted to the cover in some uniformizing chart and the isoperimetric inequality [29, Theorem 4.4.1] can be applied. The rest of the proof can be completed in the same way as the proof of the removal of singularities result for $J$-holomorphic curves in [29, Theorem 4.1.2]. We need the second paragraph of the proof of [29, Lemma 4.5.1] (this requires Stokes' theorem for orbifolds, which can be proved by passing to a cover locally using regularizing charts), followed by the proof of [29, Theorem 4.1.2]. Note that we don't require holomorphic extension of $u_{G}$ over the singularity.

Proposition 4.1 now follows in a straightforward way.

Proof. (Proof of Proposition 4.1) Map $\mathbb{C} \backslash B_{1}$ to the half cylinder $\Sigma$, set $a=2 \pi$ and apply change of coordinates $\Sigma \ni z \mapsto e^{z} \in \mathbb{C} \backslash B_{1}$. Equip $\Sigma$ with the pullback metric. 
By Proposition 5.4, $u_{G}(\infty):=\lim _{s \rightarrow \infty} u_{G}(s, t)$ exists. Let $x \in \pi_{G}^{-1}\left(u_{G}(\infty)\right)$, and let $S$ be a slice for the $G$-action at $x$. This means $G \times{ }_{G_{x}} S \rightarrow X^{\mathrm{ss}}$ is a diffeomorphism onto its image. The map $\pi: G \times S \rightarrow X^{\text {ss }}$ is a $\left|G_{x}\right|$-cover, equip $G \times S$ with the metric $\pi^{*} \omega_{X}$. The left $K$-action is free and has moment map $\pi^{*} \Phi$. The number $n$ divides $G_{x}$, so for some large $s_{0}, u\left(\Sigma_{s>s_{0}}\right) \subseteq G S$ and the map $u$ lifts to $\tilde{u}: \tilde{\Sigma}_{s>s_{0}} \rightarrow G \times S$. Here $\tilde{\Sigma}_{s>s_{0}}=\left\{(s, t): s \geq s_{0}, t \in \mathbb{R} / 2 \pi n \mathbb{Z}\right\}$ is an $n$-cover of $\Sigma_{s>s_{0}}$ equipped with the pullback metric. Now, Proposition 5.3 can be applied to the lift $(\tilde{A}, \tilde{u}): \tilde{\Sigma}_{s \geq s_{0}} \rightarrow$ $G \times S$, and this proves Proposition 4.1.

Proof. (Proof of Proposition 4.2) We work in cylindrical co-ordinates on the complement of a ball. Map $\mathbb{C} \backslash B_{1}$ to the half cylinder $\Sigma=\{(s, t): s \geq 0, t \in \mathbb{R} / 2 \pi \mathbb{Z}\}$, with change of coordinates $\Sigma \ni z \mapsto e^{z} \in \mathbb{C} \backslash B_{1}$. The Euclidean metric on $\mathbb{C} \backslash B_{1}$ pulls back to $\omega_{\Sigma}=e^{2 s} \mathrm{~d} s \wedge \mathrm{d} t$ on $\Sigma$. The connection $A$ can be put in radial gauge, so that on $\Sigma, A=\mathrm{D}+a(s, t) d t$. The proof now is exactly same as the proof of Proposition D.7 (B) in [50]. The only asymptotic result used in that proof is the following: for some $\delta>0$,

$$
\sup _{t}\left(\left|\frac{\partial}{\partial s} u(s, t)\right|+e^{\delta s}|\Phi(u(s, t))|\right) \leq e^{-\delta s} \quad \forall s \geq 0 .
$$

In our case, by Proposition 4.1 we have this result for $\delta=\frac{2}{n}-\epsilon$. The conclusion of Proposition D.7 (B) consists of (38) in the form:

$$
\left\|k_{0}^{-1} \partial_{\theta} k_{0}+a(r, \cdot)\right\|_{L^{p}([0,2 \pi], K)}<c r^{\left(-1+\frac{2}{p}+\frac{\delta}{2}\right)} .
$$

To obtain our result, we choose $\epsilon=2\left(\frac{1}{n}-1+\frac{2}{p}-\gamma\right)$.

\section{Some AnAlytic ReSUlts}

In this section, we collect some analytic results used in the proof. The first two results are standard. The following is Proposition A.3.4 in [29].

Proposition 6.1. (Implicit function theorem) Let $F: X \rightarrow Y$ be a differentiable map between Banach spaces. Suppose that $D F(0)$ is surjective and has a right inverse $Q$, with $\|Q\| \leq C$. For all $x \in B_{\delta},\|D F(x)-D F(0)\|<\frac{1}{2 C}$. If $\|F(0)\|<\frac{\delta}{4 C}$, then $F(x)=0$ has a unique solution in $B_{\delta}$ satisfying $x \in \operatorname{Im} Q$.

Proposition 6.2. (Sobolev multiplication) Let $\Sigma$ be a compact $n$-dimensional manifold possibly with a smooth boundary.

(a) ([1, Theorem 4.39]) Given $k \in \mathbb{Z}$ and $p>1$ be such that $k p>n$. Then, $W^{k, p}(\Sigma)$ is a Banach algebra with respect to pointwise multiplication. There is a consant $c$ such that for any $f, g \in W^{k, p}(\Sigma)$

$$
\|f g\|_{W^{k, p}} \leq c\|f\|_{W^{k, p}}\|g\|_{W^{k, p}} .
$$

(b) ([35, Theorem $9.5(3)])$ Suppose $l \in \mathbb{Z}, k_{i} \in \mathbb{Z}_{\geq 0}$ and $q, p_{i}>1$, where $i=1,2$. Suppose at least for one $i, k_{i} p_{i}<n$. Let $l \leq k_{1}, k_{2}$ and $l-\frac{n}{q}<\sum_{i=1,2} k_{i}-\frac{n}{p_{i}}$. 
Further, if $l<0$, then we assume $\sum_{i: k_{i} p_{i}<n}\left(\frac{n}{p_{i}}-k_{i}\right)<n$. Then, there is a consant $c$ such that for any $f \in W^{k_{1}, p_{1}}(\Sigma), g \in W^{k_{2}, p_{2}}(\Sigma)$

$$
\|f g\|_{W^{l, q}} \leq c\|f\|_{W^{k_{1}, p_{1}}}\|g\|_{W^{k_{2}, p_{2}}} .
$$

The following result is Theorem 1 in [16] applied to the case where the base manifold is a Riemann surface.

Theorem 6.3. (Donaldson [16], Theorem 1) Let $\Sigma$ be a compact Riemann surface with boundary, and let $A$ be a $H^{1}$ connection on the trivial bundle $\Sigma \times K$. There is a unique $s \in H^{2}(\Sigma, \mathfrak{k})$ satisfying $\left.s\right|_{\partial \Sigma} \equiv 0$ and $e^{i s} A$ is a flat connection.

Lemma 6.4. (Action of $\mathcal{G}_{\mathbb{C}}$ on $\left.\mathcal{A}\right)$ Let $\left(\Sigma, j_{\Sigma}\right)$ be a compact Riemann surface, possibly with a smooth boundary and $P$ be a principal $K$-bundle. Let $k \in \mathbb{Z}_{\geq 0}, p>1$ be such that $(k+1) p>2$. Complex gauge transformations in $\mathcal{G}^{k+1, p}(P)$ act smoothly on the space of connections $\mathcal{A}^{k, p}(P)$.

Let $A_{0}$ be a smooth connection on $P$. For any $\epsilon>0$, there is a constant $C$ so that the following is satisfied. For any $W^{k, p}$ connection $A=A_{0}+a$ which satisfies $\|a\|_{W^{k, p}(\Sigma)}<\epsilon$ and any $\xi \in W^{k+1, p}(\Sigma, P(\mathfrak{k}))$ that satisfies $\|\xi\|_{W^{k+1, p}}<1$,

$$
\|(\exp i \xi) A-A\|_{W^{k, p}(\Sigma)} \leq C\|\xi\|_{W^{k+1, p}(\Sigma)} .
$$

Proof. The action of unitary gauge transformations $\mathcal{K}^{k+1, p}$ is smooth on $\mathcal{A}^{k, p}$ - this is a standard result, see [45, Lemma A.5]. To prove the same for $\mathcal{G}^{k+1, p}$, it is enough to look at complex gauge transformations of the form $e^{i \xi}$, where $\xi \in W^{k+1, p}(\Sigma, P(\mathfrak{k}))$. The infinitesimal action of $i \xi$ on a connection $A$ is $\mathrm{d}_{A} \xi \circ j_{\Sigma}$. Suppose $a_{t}:[0,1] \rightarrow$ $W^{k, p}(\Sigma, P(\mathfrak{k}))$ is the solution of the equation

$$
\frac{d a_{t}}{d t}=\left(\mathrm{d}_{A+a_{t}} \xi\right) \circ j_{\Sigma}=\left(\mathrm{d}_{A_{0}} \xi+\left[a_{t}, \xi\right]\right) \circ j_{\Sigma}, \quad a_{0}=a .
$$

Then, $(\exp i \xi) A=A_{0}+a_{1}$. The map

$$
W^{k, p} \times W^{k+1, p} \ni(a, \xi) \mapsto\left(\mathrm{d}_{A_{0}} \xi+[a, \xi]\right) \circ j_{\Sigma} \in W^{k, p}
$$

is smooth. So, the equation (51) has a solution $t \mapsto a_{t}$ on the time interval $[0,1]$. Further $a_{1}$ varies smoothly with $a_{0}$ and $\xi$. This shows that $\mathcal{G}^{k+1, p}$ has a smooth action on $\mathcal{A}^{k, p}$.

Next, we prove the norm bound assuming $\|\xi\|_{W^{k+1, p}}<1$. Suppose $a_{t} \neq 0$ for all $t \in[0,1]$. Then,

$$
\frac{d}{d t}\left\|a_{t}\right\|_{W^{k, p}} \leq\left\|\frac{d a_{t}}{d t}\right\|_{W^{k, p}} \leq c\left(\|\xi\|_{W^{k+1, p}}+\left\|a_{t}\right\|_{W^{k, p}}\|\xi\|_{W^{k+1, p}}\right) .
$$

We remark that $\frac{d}{d t}\left\|a_{t}\right\|_{W^{k, p}}$ is well-defined since $t \mapsto a_{t}$ is differentiable and $a_{t}$ is not identically zero for any $t \in[0,1]$. Since $\|\xi\|_{W^{k+1, p}}<1$, we have $\frac{d}{d t}\left\|a_{t}\right\|_{W^{k, p}} \leq$ $c\left(1+\|a\|_{W^{k, p}}\right)$. Then,

$$
\left\|a_{t}\right\|_{W^{k, p}} \leq e^{c t}\left(1+\left\|a_{0}\right\|_{W^{k, p}}\right)-1 \leq C(\epsilon) .
$$

Now, we use (52) again and write $\frac{d}{d t}\left\|a_{t}\right\|_{W^{k, p}} \leq C\|\xi\|_{W^{k+1, p}}$. This proves the result if $a_{t} \neq 0$ for all $t \in[0,1]$. If that is not the case, we apply the procedure on 
the intervals $\left[0, t_{0}\right]$ and $\left[t_{1}, 1\right]$, where $t_{0}, t_{1}$ are the smallest and largest time values for which $\left\|a_{t}\right\| \leq \epsilon$. This would produce the same bound.

Lemma 6.5. (Converging connections are related by converging gauge transformations) Let $\Sigma \subset \mathbb{C}$ be the closure of a bounded open subset with smooth boundary and let $P:=\Sigma \times K$ be the trivial principal bundle over $\Sigma$. Suppose $p>2$ and $A_{i}$ is a sequence of connections converging to $A_{\infty}$ in $L^{p}$. Suppose furthermore that the connections in the sequence are gauge equivalent, i.e. there exist $k_{i} \in W^{1, p}(\Sigma, K)$ such that $k_{i}\left(A_{0}\right)=A_{i}$. Then, there exists $k_{\infty} \in W^{1, p}(\Sigma, K)$ such that, after passing to a subsequence, $k_{i} \rightarrow k_{\infty}$ weakly in $W^{1, p}$. Further, $A_{\infty}=k_{\infty}\left(A_{0}\right)$ and so the limit is in the same gauge orbit as the sequence.

Proof. This result is a slight variation of Lemma 4.3.5 in [43]. The connections can be written as $A_{i}=\mathrm{D}+a_{i}$, where $a_{i} \in \Omega^{1}(\Sigma, \mathfrak{k})$. Any compact Lie group injectively embeds into $U(N)$. After fixing such an embedding, we can use matrix representations for $a_{i}, k_{i}$ etc. The relation $k_{i} A_{0}=A_{i}$ can be re-written as

$$
a_{i}=\left(d k_{i}\right) k_{i}^{-1}+k_{i} a_{0} k_{i}^{-1} \text {. }
$$

Let $\Theta_{i}:=A_{i}-A_{\infty}$. Then,

$$
d k_{i}=-k_{i} a_{0}+a_{\infty} k_{i}+\Theta_{i} k_{i} .
$$

The one-forms $A_{0}, A_{\infty}$ and $\Theta_{i}$ are bounded in $L^{p}$. We can assume that the norm on the space of $N \times N$ matrices is invariant under left and right multiplication by elements in $K$. Then, the right hand side of (53) is bounded in $L^{p}$. Since $K$ is compact, $\left\|k_{i}\right\|_{W^{1, p}}<c$ for some constant $c$. After passing to a subsequence, $k_{i} \rightarrow k_{\infty}$ in $W^{1, p}$. So, the sequence $k_{i} A_{0}$, which is equal to the sequence $A_{i}$, converges to $k_{\infty} A_{0}$ weakly in $L^{p}$. The limit must be equal to the strong limit, which is $A_{\infty}$.

\section{REFERENCES}

11] R. A. Adams, Sobolev spaces, Academic Press [A subsidiary of Harcourt Brace Jovanovich, Publishers], New York-London, 1975, Pure and Applied Mathematics, Vol. 65. MR 0450957 (56 \#9247)

[2] A. Adem, J. Leida, and Y. Ruan. Orbifolds and stringy topology, volume 171 of Cambridge Tracts in Mathematics. Cambridge University Press, Cambridge, 2007.

[3] A. Bertram, I. Ciocan-Fontanine, and B. Kim, Two proofs of a conjecture of Hori and Vafa, Duke Math. J. 126 (2005), no. 1, 101-136. MR 2110629 (2006e:14077)

[4] I. Biswas, A. Dhillon, J. Hurtubise, and R. Wentworth. A generalized Quot scheme and meromorphic vortices. ArXiv e-prints, October 2014.

[5] S. Bradlow and G. Daskalopoulos, Moduli of stable pairs for holomorphic bundles over Riemann surfaces. II, Internat. J. Math. 4 (1993), no. 6, 903-925. MR 1250254 (96c:32020)

[6] S. Bradlow, Vortices in holomorphic line bundles over closed Kähler manifolds, Comm. Math. Phys. 135 (1990), no. 1, 1-17. MR 1086749 (92f:32053)

[7] _ Special metrics and stability for holomorphic bundles with global sections, J. Differential Geom. 33 (1991), no. 1, 169-213. MR 1085139 (91m:32031)

[8] S. Bradlow and G. Daskalopoulos, Moduli of stable pairs for holomorphic bundles over Riemann surfaces, Internat. J. Math. 2 (1991), no. 5, 477-513. MR 1124279 (93b:58026)

[9] W. Chen and Y. Ruan, Orbifold Gromov-Witten theory, Orbifolds in mathematics and physics (Madison, WI, 2001), Contemp. Math., vol. 310, Amer. Math. Soc., Providence, RI, 2002, pp. 25-85. MR 1950941 (2004k:53145) 
[10] K. Cieliebak, A. Rita Gaio, I. Mundet i Riera, and D. Salamon, The symplectic vortex equations and invariants of Hamiltonian group actions, J. Symplectic Geom. 1 (2002), no. 3, 543-645. MR 1959059 (2004g:53098)

[11] K. Cieliebak, A. Gaio, and D. Salamon, J-holomorphic curves, moment maps, and invariants of Hamiltonian group actions, Internat. Math. Res. Notices (2000), no. 16, 831-882. MR MR1777853 (2001h:53130)

[12] M. Cornalba and P. Griffiths, Analytic cycles and vector bundles on non-compact algebraic varieties, Invent. Math. 28 (1975), 1-106. MR 0367263 (51 \#3505)

[13] T. Dimofte, S. Gukov, and L. Hollands, Vortex counting and Lagrangian 3-manifolds, Lett. Math. Phys. 98 (2011), no. 3, 225-287. MR 2852983

[14] S. K. Donaldson, A new proof of a theorem of Narasimhan and Seshadri, J. Differential Geom. 18 (1983), no. 2, 269-277. MR MR710055 (85a:32036)

[15] _ Anti self-dual Yang-Mills connections over complex algebraic surfaces and stable vector bundles, Proc. London Math. Soc. (3) 50 (1985), no. 1, 1-26. MR MR765366 (86h:58038)

[16] B Boundary value problems for Yang-Mills fields, J. Geom. Phys. 8 (1992), no. 1-4, 89-122. MR 1165874 (93d:53033)

[17] Y. Eliashberg and M. Gromov, Convex symplectic manifolds, Several complex variables and complex geometry, Part 2 (Santa Cruz, CA, 1989), Proc. Sympos. Pure Math., vol. 52, Amer. Math. Soc., Providence, RI, 1991, pp. 135-162. MR 1128541 (93f:58073)

[18] A. Gaio and D. Salamon, Gromov-Witten invariants of symplectic quotients and adiabatic limits, J. Symplectic Geom. 3 (2005), no. 1, 55-159. MR 2198773 (2007k:53156)

[19] O. Garcia-Prada. Dimensional reduction of stable bundles, vortices and stable pairs. Internat. J. Math. 5 (1994), no. 1, 1-52.

[20] O. Garcia-Prada. A direct existence proof for the vortex equations over a compact Riemann surface. Bull. London Math. Soc. 26 (1994), no. 1, 88-96.

[21] O. Garcia-Prada. Invariant connections and vortices. Comm. Math. Phys. 156 (1993), no. 3, $527-546$.

[22] A. Grothendieck, Sur la classification des fibrés holomorphes sur la sphère de Riemann, Amer. J. Math. 79 (1957), 121-138. MR 19,315b

[23] S. Helgason. Differential geometry, Lie groups, and symmetric spaces, volume 80 of Pure and Applied Mathematics. Academic Press Inc. [Harcourt Brace Jovanovich Publishers], New York, 1978.

[24] A. Jaffe and C. Taubes, Vortices and monopoles, Progress in Physics, vol. 2, Birkhäuser Boston, Mass., 1980, Structure of static gauge theories. MR 614447 (82m:81051)

[25] G. Kempf and L. Ness, The length of vectors in representation spaces, Algebraic geometry (Proc. Summer Meeting, Univ. Copenhagen, Copenhagen, 1978), Lecture Notes in Math., vol. 732, Springer, Berlin, 1979, pp. 233-243. MR MR555701 (81i:14032)

[26] A. D. King, Moduli of representations of finite-dimensional algebras, Quart. J. Math. Oxford Ser. (2) 45 (1994), no. 180, 515-530. MR 1315461 (96a:16009)

[27] F. C. Kirwan, Cohomology of quotients in symplectic and algebraic geometry, Mathematical Notes, vol. 31, Princeton University Press, Princeton, NJ, 1984. MR MR766741 (86i:58050)

[28] E. Lerman, Orbifolds as stacks?, Enseign. Math. (2) 56 (2010), no. 3-4, 315-363. MR 2778793 (2012c:18010)

[29] D. McDuff and D. Salamon, J-holomorphic curves and symplectic topology, American Mathematical Society Colloquium Publications, vol. 52, American Mathematical Society, Providence, RI, 2004. MR 2045629 (2004m:53154)

[30] D. Mumford, J. Fogarty, and F. Kirwan, Geometric invariant theory, third ed., Ergebnisse der Mathematik und ihrer Grenzgebiete (2) [Results in Mathematics and Related Areas (2)], vol. 34, Springer-Verlag, Berlin, 1994. MR 1304906 (95m:14012)

[31] I. Mundet i Riera, A Hitchin-Kobayashi correspondence for Kähler fibrations, J. Reine Angew. Math. 528 (2000), 41-80. MR MR1801657 (2002b:53035)

[32] M. S. Narasimhan and C. S. Seshadri, Stable and unitary vector bundles on a compact Riemann surface, Ann. of Math. (2) 82 (1965), 540-567. MR MR0184252 (32 \#1725) 
[33] K. L. Nguyen, C. Woodward, and F. Ziltener, Morphisms of CohFT algebras and quantization of the Kirwan map, ArXiv e-prints (2009).

[34] A. Ott. Removal of singularities and gromov compactness for symplectic vortices. J. Symplectic Geom., 12(2):257-311, 062014.

[35] R. Palais. Foundations of global non-linear analysis. W. A. Benjamin, Inc., New YorkAmsterdam, 1968.

[36] I. Satake, The Gauss-Bonnet theorem for V-manifolds, J. Math. Soc. Japan 9 (1957), 464-492. MR 0095520 (20 \#2022)

[37] I. M. Singer. The geometric interpretation of a special connection, Pacific J. Math. 9 (1959), 585-590.

[38] S. P. Smith, Computation of the Grothendieck and Picard groups of a toric DM stack X by using a homogeneous coordinate ring for X. Glasg. Math. J. 53 (2011), 97-113.

[39] E. M. Stein and R. Shakarchi. Complex analysis. Princeton Lectures in Analysis, II. Princeton University Press, Princeton, NJ, 2003.

[40] C. H. Taubes. Arbitrary $n$-vortex solutions to the first order ginzburg-landau equations. Comm. Math. Phys., 72(3):277-292, 1980.

[41] K. Uhlenbeck and S.-T. Yau, On the existence of Hermitian-Yang-Mills connections in stable vector bundles, Comm. Pure Appl. Math. 39 (1986), no. S, suppl., S257-S293, Frontiers of the mathematical sciences: 1985 (New York, 1985). MR MR861491 (88i:58154)

[42] K. Uhlenbeck, Connections with $L^{p}$ bounds on curvature, Comm. Math. Phys. 83 (1982), no. 1, 31-42. MR 648356 (83e:53035)

[43] S. Venugopalan, Yang-Mills heat flow on gauged holomorphic maps, ArXiv e-prints (2012).

[44] K. Wehrheim, Banach space valued Cauchy-Riemann equations with totally real boundary conditions, ArXiv Mathematics e-prints (2004).

[45] K. Wehrheim, Uhlenbeck compactness, EMS Series of Lectures in Mathematics, European Mathematical Society (EMS), Zürich, 2004. MR 2030823 (2004m:53045)

[46] C. Woodward, Quantum Kirwan morphism and Gromov-Witten invariants of quotients $I$. Transformation Groups, 20(2):507-556, 2015.

[47] C. Woodward, Quantum Kirwan morphism and Gromov-Witten invariants of quotients II. Transformation Groups, to appear.

[48] C. T. Woodward, Quantum Kirwan morphism and Gromov-Witten invariants of quotients III. Transformation Groups, to appear.

[49] G. Xu, U(1)-vortices and quantum Kirwan map, ArXiv e-prints (2012).

[50] F. Ziltener, Symplectic vortices on the complex plane and quantum cohomology, Ph.D. thesis, ETH Zurich, 2006.

[51] F. Ziltener, A Quantum Kirwan Map: Bubbling and Fredholm Theory for Symplectic Vortices over the Plane, Memoirs of the American Mathematical Society, 2014.

[52] F. Ziltener, The invariant symplectic action and decay for vortices, J. Symplectic Geom. 7 (2009), no. 3, 357-376. MR 2534190 (2011b:53217) 